\title{
PIECEWISE DETERMINISTIC MARKOV PROCESSES AND THEIR INVARIANT MEASURES
}

\author{
ALAIN DURMUS, ARNAUD GUILLIN, PIERRE MONMARCHÉ
}

\begin{abstract}
Piecewise Deterministic Markov Processes (PDMPs) are studied in a general framework. First, different constructions are proven to be equivalent. Second, we introduce a coupling between two PDMPs following the same differential flow which implies quantitative bounds on the total variation between the marginal distributions of the two processes. Finally two results are established regarding the invariant measures of PDMPs. A practical condition to show that a probability measure is invariant for the associated PDMP semi-group is presented. In a second time, a bound on the invariant probability measures in $V$-norm of two PDMPs following the same differential flow is established. This last result is then applied to study the asymptotic bias of some non-exact PDMP MCMC methods.
\end{abstract}

\section{INTRODUCTION}

Piecewise Deterministic Markov Processes (PDMP), similarly to diffusion processes, form an important class of Markov processes, which are used to model random dynamical systems in various fields (see e.g. $[18,1]$ ). Recently, interest has grown for their use to sample from a target distribution $[4,22,5]$. The resulting class of algorithms is referred to as PDMP Monte Carlo (PDMP-MC) methods. To this end, natural questions arise as to the stationarity of the target measure, the ergodicity of the corresponding process and possible bias introduced by the method. In mathematical physics [6] and biology [7], the long time behaviour of these processes has been the subject of several works. In this context, these studies are done through the Kolmogorov Fokker Planck operator $\mathcal{A}^{\star}$ of the PDMP of interest given for all smooth density $\rho$ on $\mathbb{R}^{2 d}$ by

$$
\mathcal{A}^{\star} \rho=-\langle\Xi, \nabla \rho\rangle+K(\lambda \rho)-\lambda \rho,
$$

where $\Xi$ is a smooth vector field of $\mathbb{R}^{2 d}, \lambda: \mathbb{R}^{2 d} \rightarrow \mathbb{R}_{+}$and $K$ is a non-local collision operator.

The relevance of the present work emerged while writing the companion paper [12], concerned with the geometric ergodicity of the Bouncy Particle Sampler (BPS) [5], an MCMC algorithm which, given a target distribution $\pi$ on $\mathbb{R}^{d}$, introduces a PDMP for which $\pi$ is invariant. In order to make rigorous several arguments in [12], technical lemmas had to be established, in particular to cope with the fact that Markov semigroups associated to PDMP lack the regularity properties of (hypo-)elliptic diffusions, which yields additional difficulties and technicalities. These results, of interest in a more general framework, are gathered here with the hope that it will set a framework where for example verification of the invariance of a measure becomes a mere calculus via the generator (as it is the case for diffusion processes under mild assumptions). To illustrate our results, BPS is used as a recurrent example. 
Let us present these different results, together with the organization of the paper. Section 2 contains the basic definitions of our framework, and in particular presents the construction of a PDMP. Alternative constructions are shown in Sections 3 and 4 to give the same process (i.e. to give a random variable with the same law on the Skorokhod space). Conditions which ensure that PDMPs are non explosive are presented in Section 5. The synchronous coupling of two PDMPs is defined in Section 6, which aims to construct simultaneously two different PDMPs, starting at the same initial state, in such a way that they have some probability to stay equal for some time. It yields estimates on the difference of the corresponding semi-groups in total variation norm. In Section 8, conditions are established under which the semi-group associated to a PDMP leaves invariant the space of compactly-supported smooth functions. Using this result, a practical criterion to ensure that a given probability measure $\mu$ is invariant for a PDMP is obtained in Section 9. Indeed, it is well-known that, denoting by $(\overline{\mathcal{A}}, \mathrm{D}(\overline{\mathcal{A}}))$ the strong generator of the Markov semi-group associated to the PDMP, then $\mu$ is invariant if and only if $\int \overline{\mathcal{A}} f \mathrm{~d} \mu=0$ for all $f$ in a core of $\overline{\mathcal{A}}$. Nevertheless, due to the lack of regularization properties of the semi-group, it is generally impossible to determine such a core. We will prove that, under some simple assumptions, it is enough to consider compactlysupported smooth functions $f$. Finally, in Section 10, we are interested in bounding the $V$-norm between two invariant probability measures $\mu_{1}$ and $\mu_{2}$ corresponding to two PDMPs sharing the same differential flow but with different jump rates and Markov kernels. This question is here mainly motivated by the thinning method used to sample trajectories of PDMPs $[17,16]$. Indeed, a PDMP can be exactly sampled (in the sense that no time discretization is needed) provided that the associated differential flow can be computed and a simple upper bound on the jump rate is known. When this is not the case, a PDMP with a truncated jump rate can be sampled, and our result gives a control on the ensuing error.

Notation and conventions. For all $a, b \in \mathbb{R}$, we denote $a_{+}=\max (0, a), a \vee b=$ $\max (a, b), a \wedge b=\min (a, b)$. Id stands for the identity matrix on $\mathbb{R}^{d}$.

For all $x, y \in \mathbb{R}^{d}$, the scalar product between $x$ and $y$ is denoted by $\langle x, y\rangle$ and the Euclidean norm of $x$ by $\|x\|$. For all $x \in \mathbb{R}^{d}, r>0$, we denote by $\mathrm{B}(x, r)=$ $\left\{w \in \mathbb{R}^{d}:\|w-x\|<r\right\}$ the ball centered at $x$ with radius $r$. The closed ball centered in $x$ with radius $r$ is denoted by $\overline{\mathrm{B}}(x, r)$. For any $d$-dimensional matrix $M$, define by $\|M\|=\sup _{w \in \mathrm{B}(0,1)}\|M w\|$ the operator norm associated with $M$.

Let $(\mathrm{M}, \mathfrak{g})$ be a smooth closed Riemannian sub-manifold of $\mathbb{R}^{N}$ and $\mathcal{B}(\mathrm{M})$ the associated Borel $\sigma$-field. Let $\infty \notin M$ be a cemetery point. The distance induced by $\mathfrak{g}$ is denoted by dist. With a slight abuse of notations, the ball (respectively closed ball) centered at $x \in \mathrm{M}$ with radius $r>0$ is denoted by $\mathrm{B}(x, r)$ (respectively $\overline{\mathrm{B}}(x, r)$ ).

For all function $F: \mathrm{M} \rightarrow \mathbb{R}^{m}$ and compact set $\mathrm{K} \subset \mathrm{M}$, denote $\|F\|_{\infty}=\sup _{x \in \mathrm{M}}\|F(x)\|$, $\|F\|_{\infty, \mathrm{K}}=\sup _{x \in \mathrm{K}}\|F(x)\|$. Denote by $\mathbb{M}(\mathrm{M})$ the space of measurable functions from $\mathrm{M}$ to $\mathbb{R}$. Denote by $B(M)$ the set of all measurable and bounded functions from $M$ to $\mathbb{R}$. The space $B(M)$ is endowed with the topology associated with the uniform norm $\|\cdot\|_{\infty}$. Let $C(M)$ stand for the set of continuous functions from $M$ to $\mathbb{R}, C_{0}(M)$ the subset of $\mathrm{C}(\mathrm{M})$ consisting of continuous functions vanishing at infinity and, for all $k \in \mathbb{N}^{*}$, let $\mathrm{C}^{k}(\mathrm{M})$ be the set of $k$-times continuously differentiable functions from $\mathrm{M}$ to $\mathbb{R}$. For all 
$k \in \mathbb{N}$, denote by $\mathrm{C}_{c}^{k}(\mathrm{M})$ and $\mathrm{C}_{b}^{k}(\mathrm{M})$ the set of functions in $\mathrm{C}^{k}(\mathrm{M})$ with compact support and the set of bounded functions in $C^{k}(M)$ respectively. For $f \in C^{k}(M)$, we denote by $\mathrm{D}^{k} f$ the $k^{\text {th }}$ differential of $f$. For all function $f: \mathrm{M} \rightarrow \mathbb{R}$, we denote by $\nabla f$ and $\nabla^{2} f$ the gradient and the Hessian of $f$ respectively, if they exist.

We denote by $\mathcal{P}(\mathrm{M})$ the set of probability measures on $\mathrm{M}$. For $\mu, \nu \in \mathcal{P}(\mathrm{M}), \xi \in \mathcal{P}\left(\mathrm{M}^{2}\right)$ is called a transference plan between $\mu$ and $\nu$ if for all $\mathrm{A} \in \mathcal{B}(\mathrm{M}), \xi(\mathrm{A} \times \mathrm{M})=\mu(\mathrm{A})$ and $\xi(\mathrm{M} \times \mathrm{A})=\nu(\mathrm{A})$. The set of transference plan between $\mu$ and $\nu$ is denoted by $\Gamma(\mu, \nu)$. The random variables $X$ and $Y$ on $\mathrm{M}$ are a coupling between $\mu$ and $\nu$ if the distribution of $(X, Y)$ belongs to $\Gamma(\mu, \nu)$. The total variation norm between $\mu$ and $\nu$ is defined by

$$
\|\mu-\nu\|_{\mathrm{TV}}=2 \inf _{\xi \in \Gamma(\mu, \nu)} \int_{\mathrm{M}^{2}} \mathbb{1}_{\Delta_{\mathrm{M}}^{c}}(x, y) \mathrm{d} \xi(x, y),
$$

where $\Delta_{\mathrm{M}}=\left\{(x, y) \in \mathrm{M}^{2}: x=y\right\}$ and $\Delta_{\mathrm{M}}^{c}=\mathrm{M}^{2} \backslash \Delta_{\mathrm{M}}$ is its complement. For all $\mu \in \mathcal{P}(\mathrm{M})$, define the support of $\mu$ by

$$
\operatorname{supp} \mu=\overline{\{x \in \mathrm{M}: \text { for all open set } \mathrm{U} \ni x, \mu(\mathrm{U})>0\}} \text {. }
$$

In the sequel, we take the convention that $\inf \emptyset=+\infty$. All the random variables considered in this paper are defined on a fixed probability space $(\Omega, \mathcal{F}, \mathbb{P})$.

\section{A first definition of Piecewise Deterministic Markov Processes}

\section{Definitions and further notation.}

Let $(\mathrm{M}, \mathfrak{g})$ be a smooth closed Riemannian sub-manifold of $\mathbb{R}^{N}$. A PDMP on $\mathrm{M}$ is defined using a triple $\left(\varphi,\left(\lambda_{i}, Q_{i}\right)_{i \in \llbracket 1, \ell \rrbracket}\right), \ell \in \mathbb{N}^{*}$, referred to as the local characteristics of a PDMP, where

- $\varphi$ is a differential flow on $\mathrm{M}: \varphi:(t, h, x) \mapsto \varphi_{t, t+h}(x)$ is a measurable function from $\mathbb{R}_{+} \times \mathbb{R}_{+} \times \mathrm{M}$ to $\mathrm{M}$, such that for all $t, h_{1}, h_{2} \geqslant 0 \varphi_{t+h_{1}, t+h_{1}+h_{2}} \circ \varphi_{t, t+h_{1}}=$ $\varphi_{t, t+h_{1}+h_{2}}, \varphi_{t, t}=\mathrm{Id}$. Moreover, for all $(t, x) \in \mathbb{R}_{+} \times \mathrm{M}, \tilde{h} \mapsto \varphi_{t, t+\tilde{h}}(x)$ is continuously differentiable from $\mathbb{R}_{+}$to $\mathrm{M}$ and for all $t, h \in \mathbb{R}_{+}, \tilde{x} \mapsto \varphi_{t, t+h}(\tilde{x})$ is a $\mathrm{C}^{1}$-diffeomorphism of $\mathrm{M}$. The flow $\varphi$ is (time-)homogeneous if for all $t, h \in \mathbb{R}_{+}$, $\varphi_{t, t+h}=\varphi_{0, h}$, in which case we set $\varphi_{h}=\varphi_{0, h}$.

- For all $i \in \llbracket 1, \ell \rrbracket, \lambda_{i}: \mathbb{R}_{+} \times \mathrm{M} \rightarrow \mathbb{R}_{+}$is a measurable function referred to as a jump rate on $M$ which is locally bounded, in the sense that $\left\|\lambda_{i}\right\|_{\infty, \mathrm{K}}<\infty$ for all compact $\mathrm{K} \subset \mathbb{R}_{+} \times \mathrm{M}$. The jump rate $\lambda_{i}$ is (time)-homogeneous if it does not depend on $t$.

- For all $i \in \llbracket 1, \ell \rrbracket, Q_{i}: \mathbb{R}_{+} \times \mathrm{M} \times \mathcal{B}(\mathrm{M}) \rightarrow[0,1]$ is an inhomogeneous Markov kernel on $\mathrm{M}$ : for all $\mathrm{A} \in \mathcal{B}(\mathrm{M}),(t, x) \mapsto Q_{i}(t, x, \mathrm{~A})$ is measurable, and for all $(t, x) \in \mathbb{R}_{+} \times \mathrm{M}, Q_{i}(t, x, \cdot) \in \mathcal{P}(\mathrm{M})$. The Markov kernel $Q_{i}$ is (time-)homogeneous if it does not depend on $t$.

In the case $\ell=1$, the local characteristics $\left(\varphi,\left(\lambda_{1}, Q_{1}\right)_{i \in \llbracket 1 \rrbracket 1}\right)$ are denoted by $\left(\varphi, \lambda_{1}, Q_{1}\right)$.

If $\varphi$ is a homogeneous differential flow and, for all $i \in \llbracket 1, \ell \rrbracket, \lambda_{i}, Q_{i}$ are homogeneous as well, the local characteristics $\left(\varphi,\left(\lambda_{i}, Q_{i}\right)_{i \in \llbracket 1, \ell \rrbracket}\right)$ are said to be homogeneous. A (homogeneous) jump mechanism on $\mathrm{M}$ is a pair $(\lambda, Q)$ constituted of a (homogeneous) jump rate and a (homogeneous) Markov kernel on $\mathrm{M}$. 
A first construction of a PDMP. For all $i \in \llbracket 1, \ell \rrbracket$, consider a representation $\mathbf{G}_{i}$ of the Markov kernel $Q_{i}$, i.e. a measurable function $\mathbf{G}_{i}: t, x, u \mapsto \mathbf{G}(t, x, u)$ from $\mathbb{R}_{+} \times \mathbf{M} \times$ $[0,1]$ to $\mathrm{M}$ such that for all $(t, x, \mathrm{~A}) \in \mathbb{R}_{+} \times \mathbf{M} \times \mathcal{B}(\mathrm{M}), Q_{i}(t, x, \mathrm{~A})=\mathbb{P}\left(\mathbf{G}_{i}(t, x, U) \in \mathrm{A}\right)$, where $U$ is a random variable uniformly distributed on $[0,1]$. By [3, Corollary 7.16.1], such a representation always exists.

Then, a PDMP $\left(X_{t}\right)_{t \geqslant 0}$ based on the local characteristics $\left(\varphi,\left(\lambda_{i}, Q_{i}\right)_{i \in \llbracket 1, \ell \rrbracket}\right)$ and the initial distribution $\mu_{0}$ can be defined recursively through a Markov chain $\left(X_{k}^{\prime}, S_{k}\right)_{k \in \mathbb{N}}$ on $(\mathrm{M} \cup\{\infty\}) \times\left(\mathbb{R}_{+} \cup\{+\infty\}\right)$. For all $k \in \mathbb{N}, X_{k}^{\prime}$ will be the state of the process $\left(X_{t}\right)_{t \geqslant 0}$ at times $S_{k}$. Between two times $S_{k}$ and $S_{k+1},\left(X_{t}\right)_{t \geqslant 0}$ will be a deterministic function of $X_{k}^{\prime}$ and $S_{k}$. More precisely, consider the following construction.

Construction 1. Let $W_{0}$ be a random variable with distribution $\mu_{0} \in \mathcal{P}(\mathrm{M})$ and $\left(\left(E_{j, k}\right)_{j \in \llbracket 1, \ell \rrbracket}, U_{k}\right)_{k \in \mathbb{N}^{*}}$ be an i.i.d. sequence, independent of $W_{0}$, such that for all $k \in \mathbb{N}^{*}$ and $j \in \llbracket 1, \ell \rrbracket, U_{k}$ is uniformly distributed on $[0,1]$ and $E_{j, k}$ is an exponential random variable with parameter 1 , independent of $U_{k}$ and from $E_{i, k}$ for $i \neq j$. Recall that $\infty \notin M$ is a cemetery point.

Set $S_{0}=0, X_{0}^{\prime}=W_{0}, I_{0}=1$ and suppose that $\left(X_{k}^{\prime}, S_{k}, I_{k}\right)$ and $\left(X_{t}\right)_{t \leqslant S_{k}}$ have been defined for some $k \in \mathbb{N}$, with $X_{k}^{\prime} \in \mathrm{M}$ and $S_{k} \in \mathbb{R}_{+}$. For all $j \in \llbracket 1, \ell \rrbracket$, set

(1) $S_{j, k+1}=\inf \left\{t \geqslant S_{k}: E_{j, k+1}<\int_{S_{k}}^{t} \lambda_{j}\left(s, \varphi_{S_{k}, s}\left(X_{k}^{\prime}\right)\right) \mathrm{d} s\right\}, S_{k+1}=\min _{j \in \llbracket 1, \ell \rrbracket} S_{j, k+1}$.

- If $S_{k+1}=+\infty$, set $S_{m}=+\infty, X_{m}^{\prime}=\infty, I_{m}=1$ for all $m>k$, and $X_{t}=$ $\varphi_{S_{k}, t}\left(X_{k}^{\prime}\right)$ for all $t \geqslant S_{k}$.

- If $S_{k+1}<+\infty$, set

$$
I_{k+1}=\min \left\{j \in \llbracket 1, \ell \rrbracket, S_{j, k+1}=S_{k+1}\right\}, X_{k+1}^{\prime}=\mathbf{G}_{I_{k+1}}\left(S_{k+1}, \varphi_{S_{k}, S_{k+1}}\left(X_{k}^{\prime}\right), U_{k+1}\right) .
$$

For $t \in\left(S_{k}, S_{k+1}\right)$, set $X_{t}=\varphi_{S_{k}, t}\left(X_{k}^{\prime}\right)$ and $X_{S_{k+1}}=X_{k+1}^{\prime}$.

For $t \geqslant \sup _{k \in \mathbb{N}} S_{k}$, set $X_{t}=\infty$.

Define for any $j \in \llbracket 1, \ell \rrbracket$, given $X_{k}, S_{k}, \psi_{j, k}(t)=\int_{S_{k}}^{t+S_{k}} \lambda_{j}\left(s, \varphi_{S_{k}, s}\left(X_{k}^{\prime}\right)\right) \mathrm{d} s$ and $\psi_{j, k}^{\overleftarrow{ }}$ its generalized inverse which is defined since $\psi_{j, k}$ is non-decreasing. Note that by Construction 1 , for $k \in \mathbb{N}, i, j \in \llbracket 1, \ell \rrbracket, i \neq j$, using properties of the generalized inverse, since $\psi_{j, k}$ is continuous, we obtain

$$
\mathbb{P}\left(S_{j, k+1}=S_{i, k+1}=S_{k+1}<+\infty \mid X_{k}, S_{k}\right)=\mathbb{P}\left(E_{j, k+1}=\psi_{j, k} \circ \psi_{i, k}^{\overleftarrow{ }}\left(E_{i, k+1}\right), S_{k+1}<+\infty\right)=0
$$

However, the definition of $I_{k+1}$ ensures that the process $\left(X_{k}^{\prime}, S_{k}\right)$ is defined not only almost everywhere on $\Omega$, but in fact on all $\Omega$.

Let $\left(\mathcal{F}_{k}^{\prime}\right)_{k \in \mathbb{N}}$ be the filtration associated with $\left(X_{k}^{\prime}, S_{k}, I_{k}\right)_{k \in \mathbb{N}}$. Then, $\left(X_{k}^{\prime}, S_{k}, I_{k}\right)_{k \in \mathbb{N}}$ is an inhomogeneous Markov chains since for all $k \in \mathbb{N}, \mathrm{A} \in \mathcal{B}(\mathrm{M}), t \geqslant S_{k}, j \in \llbracket 1, \ell \rrbracket$,

$$
\mathbb{P}\left(X_{k+1}^{\prime} \in \mathrm{A}, S_{k+1} \leqslant t, I_{k+1}=j \mid \mathcal{F}_{k}^{\prime}\right)=\mathbb{1}_{\mathrm{M}}\left(X_{k}^{\prime}\right) \int_{S_{k}}^{t} Q_{j}\left(s, \varphi_{S_{k}, s}\left(X_{k}^{\prime}\right), \mathrm{A}\right) \lambda_{j}\left(s, \varphi_{S_{k}, s}\left(X_{k}^{\prime}\right)\right)
$$




$$
\times \exp \left\{-\sum_{i=1}^{\ell} \int_{S_{k}}^{s} \lambda_{i}\left(u, \varphi_{S_{k}, u}\left(X_{k}^{\prime}\right)\right) \mathrm{d} u\right\} \mathrm{d} s .
$$

Note that the sequence $\left(X_{k}^{\prime}, S_{k}\right)_{k \in \mathbb{N}}$ is an inhomogeneous Markov chain as well, whose kernel can be straightforwardly deduced from (3).

Then, $\left(X_{t}\right)_{t \geqslant 0}$ is a stochastic process on $\mathrm{M} \cup\{\infty\}$, i.e. it is a random variable from $(\Omega, \mathcal{F}, \mathbb{P})$ to the space $\mathrm{D}\left(\mathbb{R}_{+}, \mathrm{M} \cup\{\infty\}\right)$ of càdlàg functions from $\mathbb{R}_{+}$to $\mathrm{M} \cup\{\infty\}$, endowed with the Skorokhod topology, see [15, Chapter 6]. Moreover, $\left(X_{t}\right)_{t \geqslant 0}$ is a Markov process [14, Theorem 7.3.1], from the class of piecewise deterministic Markov processes (PDMPs). We say that a stochastic process $\left(\tilde{X}_{t}\right)_{t \geqslant 0}$ is a PDMP with local characteristics $\left(\varphi,\left(\lambda_{i}, Q_{i}\right)_{i \in \llbracket 1, n \rrbracket}\right)$ and initial distribution $\mu_{0}$ if it has the same distribution on $\mathrm{D}\left(\mathbb{R}_{+}, \mathrm{M} \cup\{\infty\}\right)$ as $\left(X_{t}\right)_{t \geqslant 0}$. We will denote by $\operatorname{PDMP}\left(\varphi,\left(\lambda_{i}, Q_{i}\right)_{i \in \llbracket 1, \ell \rrbracket}, \mu_{0}\right)$ this distribution. In the sequel, we will see that a given PDMP can admit several local characteristics. Note that, as $\varphi$ is a $\mathrm{C}^{1}$-diffeomorphism, $\left(X_{t}\right)_{t \geqslant 0}$ is completely determined by the Markov chain $\left(X_{k}^{\prime}, S_{k}\right)_{k \in \mathbb{N}}$, referred to as the embedded chain associated to the process. The sequence $\left(S_{k}\right)_{k \in \mathbb{N}}$ is said to be the jump times of the process $\left(X_{t}\right)_{t \geqslant 0}$. A PDMP is said to be homogeneous if its local characteristics are (time) homogeneous.

For $\left(\tilde{X}_{t}\right)_{t \geqslant 0} \in \mathrm{D}\left(\mathbb{R}_{+}, \mathrm{M} \cup\{\infty\}\right)$, we call $\tau_{\infty}(\tilde{X})=\inf \left\{t \geqslant 0: \tilde{X}_{t}=\infty\right\}$ the explosion time of the process $\left(\tilde{X}_{t}\right)_{t \geqslant 0}$. A process $\left(\tilde{X}_{t}\right)_{t \geqslant 0}$ is said to be non-explosive if $\tau_{\infty}(\tilde{X})=+\infty$ almost surely. PDMP characteristics are said to be non-explosive if for all initial distribution the associated PDMP is non-explosive.

Construction 1 associated with the characteristics $\left(\varphi,\left(\lambda_{i}, Q_{i}\right)_{i \in \llbracket 1, \ell \rrbracket}\right.$ defines a Markov semi-group $\left(P_{s, t}\right)_{t \geqslant s \geqslant 0}$ for all $x \in \mathrm{M}, \mathrm{A} \in \mathcal{B}(\mathrm{M})$ and $t \geqslant s \geqslant 0$ by

$$
P_{s, t}(x, \mathrm{~A})=\mathbb{P}\left(\bar{X}_{t-s}^{s, x} \in \mathrm{A}\right),
$$

where $\left(\bar{X}_{u}^{s, x}\right)_{u \geqslant 0}$ is a PDMP with characteristics $\left(\left(\varphi_{s+u, s+t}\right)_{t \geqslant u \geqslant 0},\left(\lambda_{i}(s+\cdot, \cdot), Q_{i}(s+\right.\right.$ $\left.\cdot, \cdot, \cdot))_{i \in \llbracket 1, \ell \rrbracket}\right)$ starting from $x$. Its left-action on $\mathrm{C}(\mathrm{M})$ and right-action on $\mathcal{P}(\mathrm{M})$ are then given by

$$
P_{s, t} f(x)=\mathbb{E}\left[f\left(\bar{X}_{t}^{x, s}\right)\right], \quad \nu P_{s, t}(\mathrm{~A})=\int_{\mathrm{M}} \mathbb{P}\left(\bar{X}_{t}^{x, s} \in \mathrm{A}\right) \mathrm{d} \nu(x),
$$

for all $f \in \mathrm{B}(\mathrm{M}), x \in \mathrm{M}, \nu \in \mathcal{P}(\mathrm{M}), \mathrm{A} \in \mathcal{B}(\mathrm{M})$ and $t \geqslant s \geqslant 0$. The Markov property of $\left(X_{t}\right)_{t \geqslant 0}$ is equivalent to the semi-group property $P_{u, s} P_{s, t}=P_{u, t}$ for all $t \geqslant s \geqslant u \geqslant 0$. If $\left(\varphi,\left(\lambda_{i}, Q_{i}\right)_{i \llbracket 1, \ell \rrbracket}\right)$ is non explosive, then $P_{s, t}$ is a Markov kernel for all $t \geqslant s \geqslant 0$ and we say that $\left(P_{s, t}\right)_{t \geqslant s \geqslant 0}$ is non explosive. Otherwise, it is a sub-Markovian kernel only. For a homogeneous process, we simply write $P_{t}=P_{0, t}$ for all $t \geqslant 0$.

For a PDMP $\left(X_{t}\right)_{t \geqslant 0}$ with jump times $\left(S_{k}\right)_{k \in \mathbb{N}}$, we say that at time $S_{k+1}, k \geqslant 0$, a true jump occurred if $X_{S_{k+1}} \neq \varphi_{S_{k}, S_{k+1}}\left(X_{S_{k}}\right)$. Else, we say that at time $S_{k+1}$ a fantom jumped occurred. Note that, in the definition of homogeneous PDMPs with characteristics $(\varphi, \lambda, Q)$ given in [9, standard conditions p. 62], fantom jumps are impossible, since it is assumed that for all $x \in \mathrm{M}, Q(x,\{x\})=0$. This is not the case with the definition we gave in Section 2, where the notion of jump times depends on the jump mechanisms used to define the process. We will see that in Section 4 that under our settings, based on characteristics $\left(\varphi,\left(\lambda_{i}, Q_{i}\right)_{i \in \llbracket 1, \ell \rrbracket}\right)$ which define a PDMP $\left(X_{t}\right)_{t \geqslant 0}$, we can always define some characteristics $(\varphi, \lambda, Q)$ which define a $\operatorname{PDMP}\left(Z_{t}\right)_{t \geqslant 0}$ with the same distribution as $\left(X_{t}\right)_{t \geqslant 0}$ but no fantom jump. 
The condition imposed by [9] implying that a PDMP has no fantom jump can be very useful since it allows a one-to-one correspondence between the path of the continuoustime process $\left(X_{t}\right)_{t \geqslant 0}$ and of its embedded chain $\left(X_{k}^{\prime}, S_{k}\right)_{k \in \mathbb{N}}$. With our construction, the continuous process is completely determined by its embedded chain but not the opposite.

On the other hand, adding fantom jumps sometimes turns out to be convenient. Here is an example: let $(\varphi, \lambda, Q)$ be the characteristics of a PDMP $\left(X_{t}\right)_{t \geqslant 0}$, and suppose that there exists $\lambda_{*}>0$ such that $\lambda(t, x) \leqslant \lambda_{*}$ for all $t \geqslant 0$ and $x \in \mathrm{M}$. From Theorem 5 below, $\left(X_{t}\right)_{t \geqslant 0}$ has the same distribution as the PDMP $\left(Z_{t}\right)_{t \geqslant 0}$ obtained through Construction 1 from the characteristics $\left(\varphi, \lambda_{*}, \tilde{Q}\right)$ with for all $(t, x, \mathrm{~A}) \in \mathbb{R}_{+} \times \mathrm{M} \times \mathcal{B}(\mathrm{M})$,

$$
\tilde{Q}(t, x, \mathrm{~A})=\frac{\lambda(t, x)}{\lambda_{*}} Q(t, x, \mathrm{~A})+\left\{1-\frac{\lambda(t, x)}{\lambda_{*}}\right\} \delta_{x}(\mathrm{~A}) .
$$

The jump times of $\left(Z_{t}\right)_{t \geqslant 0}$ are given by a Poisson process with intensity $\lambda_{*}$. Adding fantom jumps to PDMPs turns our to be very useful in practice for their simulation. This method is referred to as thinning (see [16] and references therein for more details). Note that fantom jumps and the link between the process and the embedded chain are also investigated for a different type of PDMPs in [2].

Another use of fantom jump is presented in [8]. The stability or ergodicity of a $\operatorname{PDMP}\left(X_{t}\right)_{t \geqslant 0}$ and of its embedded chain $\left(X_{k}^{\prime}, T_{k}\right)_{k \in \mathbb{N}}$ may differ, but this is no more the case if fantom jumps are added at constant rate, i.e. if we consider the PDMP with characteristics $(\varphi, \lambda+1, \tilde{Q})$, where $\tilde{Q}$ is given for all $(t, x, \mathrm{~A}) \in \mathbb{R}_{+} \times \mathrm{M} \times \mathcal{B}(\mathrm{M})$ by

$$
\tilde{Q}(t, x, \mathrm{~A})=\frac{\lambda(t, x)}{1+\lambda(t, x)} Q(t, x, \mathrm{~A})+\frac{1}{1+\lambda(t, x)} \delta_{x}(\mathrm{~A})
$$

and its embedded chain. See [8] for more details.

There are other differences between the assumptions we consider on the characteristics of a PDMP and those in [9, standard conditions p. 62]. First, in contrast to [9], we consider that the differential flow $\left(\varphi_{t}\right)_{t \geqslant 0}$ is defined on $\mathbb{R}_{+}$. In addition, the jump rates $\left(\lambda_{i}\right)_{1 \in \llbracket 1, \ell \rrbracket}$ are supposed to be locally bounded to prevent some artificial pathological behavior such as an infinite number of fantom jumps in a finite time. However, the following weaker condition would have been sufficient to define $\left(X_{t}\right)_{t \geqslant 0}$ : for all $(t, x) \in$ $\mathbb{R}_{+} \times \mathrm{M}$, there exists $h>0$ such that $\int_{t}^{t+h} \lambda\left(s, \varphi_{t, s}(x)\right) \mathrm{d} s<+\infty$. On the other hand, we don't assume a priori that PDMPs are non-explosive.

\section{Examples.}

Several examples of PDMP can be found in [18] and references therein. In the present paper, a particular attention will be paid to the family of velocity jump PDMP, described as follows. Let $\mathrm{V} \subset \mathbb{R}^{d}$ be a smooth complete Riemannian submanifold, and set $\mathrm{M}=$ $\mathbb{R}^{d} \times \mathrm{V}$. Then, $\mathrm{M}$ is a smooth complete Riemannian submanifold of $\mathbb{R}^{2 d}$ endowed with the canonical Euclidean distance and tensor metric. We say that a $\operatorname{PDMP}\left(X_{t}, Y_{t}\right)_{t \geqslant 0}$ on $\mathrm{M}$ (where $X_{t} \in \mathbb{R}^{d}$ and $Y_{t} \in \mathrm{V}$ for all $\left.t \geqslant 0\right)$ with characteristics $\left(\varphi,\left(\lambda_{i}, Q_{i}\right)_{i \in \llbracket 1, \ell \rrbracket}\right)$ is a velocity jump PDMP if $\varphi$ is homogeneous and given for any $t \in \mathbb{R}_{+}$and $(x, v) \in \mathbb{R}_{+} \times \mathrm{V}$ by

$$
\varphi_{t}(x, y)=(x+t y, y)
$$


and if for all $i \in \llbracket 1, \ell \rrbracket$, all $\mathrm{A} \in \mathcal{B}\left(\mathbb{R}^{d}\right)$ and all $(t, x, y) \in \mathbb{R}_{+} \times \mathbb{R}^{d} \times \mathrm{V}$,

$$
Q_{i}(t,(x, y), \mathrm{A} \times \mathrm{V})=\delta_{x}(\mathrm{~A}) .
$$

Consider the PDMP $\left(X_{t}, Y_{t}\right)_{t \geqslant 0}$ associated with this choice of characteristics and $\left(X_{k}^{\prime}, Y_{k}^{\prime}, S_{k}\right)_{k \geqslant 0}$ the corresponding embedded chain. Note that by construction for all $t \in\left[S_{k}, S_{k+1}\right), k \in \mathbb{N}, X_{t}=X_{k}^{\prime}+\left(t-S_{k}\right) Y_{k}^{\prime}$ and $Y_{t}=Y_{k}^{\prime}$. Therefore for all $t<\sup _{k \in \mathbb{N}} S_{k}$, $X_{t}=\int_{0}^{t} Y_{s} \mathrm{~d} s$ and only $\left(Y_{t}\right)_{t \geqslant 0}$ can be discontinuous in time.

The class of velocity jump processes gathers the Zig-Zag process [4], the Bouncy Particle Sampler (BPS) [22] and many of their variants. The choice for $\left(\lambda_{i}, Q_{i}\right), i \in$ $\{1, \ldots, \ell\}$, of these different (but similar) processes are mainly of one of the following type (here we only consider homogeneous mechanisms):

- refreshment mechanism: the rate $\lambda(x, y)$ only depends on $x \in \mathbb{R}^{d}$, and the kernel $Q$ is constant, i.e. there exists $\nu \in \mathcal{P}(\mathrm{V})$ such that for all $(x, y) \in \mathbb{R}^{d} \times \mathrm{V}$ and all $\left(\mathrm{A}, \mathrm{A}^{\prime}\right) \in \mathcal{B}(\mathrm{M}) \times \mathcal{B}(\mathrm{V})$

$$
Q\left((x, y), \mathrm{A} \times \mathrm{A}^{\prime}\right)=\delta_{x}(\mathrm{~A}) \nu\left(\mathrm{A}^{\prime}\right) .
$$

- deterministic bounce mechanism: there exists a measurable function $g: \mathbb{R}^{d} \rightarrow$ $\mathbb{R}^{d}$, locally bounded, such that for all $(x, y) \in \mathrm{M}, \lambda(x, y)=\langle g(x), y\rangle_{+}$and $Q((x, y),\{x\} \times\{\mathrm{R}(x, y)\})=1$, for a measurable function $\mathrm{R}: \mathrm{M} \rightarrow \mathrm{Y}$. A particular example in the case $\mathrm{Y}=\mathrm{S}^{d}$ or $\mathrm{Y}=\mathbb{R}^{d}$ and $\mathrm{R}$ is given for all $(x, y) \in \mathrm{M}$ by

$$
\mathrm{R}(x, y)= \begin{cases}y-2\|g(x)\|^{-2}\langle g(x), y\rangle g(x) & \text { if } g(x) \neq 0 \\ y & \text { otherwise }\end{cases}
$$

Note that $\mathrm{R}(x, y)$ is simply the orthogonal reflection of $y$ with respect to $g(x)$ if $g(x) \neq 0$.

- randomized bounce mechanism: there exists a measurable function $g: \mathbb{R}^{d} \rightarrow \mathbb{R}^{d}$ such that for all $(x, y) \in \mathrm{M}$ and $\mathrm{A} \in \mathcal{B}\left(\mathbb{R}^{d}\right), \mathrm{A}^{\prime} \in \mathcal{B}(\mathrm{Y}), \lambda(x, y)=\langle g(x), y\rangle_{+}$and $Q\left((x, y), \mathrm{A} \times \mathrm{A}^{\prime}\right)=\delta_{x}(\mathrm{~A}) \tilde{Q}\left((x, y), \mathrm{A}^{\prime}\right)$, where $\tilde{Q}$ is a Markov kernel on $\mathrm{M} \times \mathcal{B}(\mathrm{Y})$.

For instance, [6] studies the velocity jump process associated with the linear Boltzmann equation, which gives an exemple of refreshment mechanism. The Zig-Zag (ZZ) process [4] and the Bouncy Particle Sampler (BPS) [22, 21, 10] are recently proposed PDMP used to sample from a target density $\pi \propto \exp (-U)$, where $U: \mathbb{R}^{d} \rightarrow \mathbb{R}$ is a continuously differentiable function. The $\mathrm{ZZ}$ process is a velocity jump process with $\mathrm{Y}=\{-1,1\}^{d}$ and $d$ deterministic bounce mechanisms $\left(\lambda_{i}, Q_{i}\right)_{i \in \llbracket 1, d \rrbracket}$ given for all $i \in \llbracket 1, d \rrbracket, x \in \mathbb{R}^{d}, y \in\{-1,1\}^{d}$ and $\mathrm{A} \in \mathcal{B}\left(\mathbb{R}^{d}\right)$ by $\lambda_{i}(x, y)=\left(y_{i} \partial U(x) / \partial x_{i}\right)_{+}, \quad Q_{i}\left((x, y),\{x\} \times\left\{\left(y_{1}, \ldots, y_{i-1},-y_{i}, y_{i+1}, \ldots, y_{d}\right)\right\}\right)=1$. Note that in this case, for all $x \in \mathbb{R}^{d}, g_{i}(x)=\left(\partial U(x) / \partial x_{i}\right) \mathbf{e}_{i}$ where $\mathbf{e}_{i}$ is the $i^{\text {th }}$ vector of the standard basis of $\mathbb{R}^{d}$. Additional refreshment mechanisms can be added to the process. In the rest of this paper, we will repeatedly use the BPS process as an illustration to our different results.

Example-Bouncy Particle Sampler 1. Let $\vee$ be a smooth closed sub-manifold of $\mathbb{R}^{d}$ rotation invariant, i.e. for any rotation $O$ of $\mathbb{R}^{d}, O \mathrm{~V}=\mathrm{V}$. Let $\lambda_{\mathrm{c}}>0$ and $\mu_{\mathrm{v}} \in \mathcal{P}(\mathrm{V})$. The BPS process associated with the potential $U$, refreshment rate $\lambda_{\mathrm{c}}$ and refreshment 


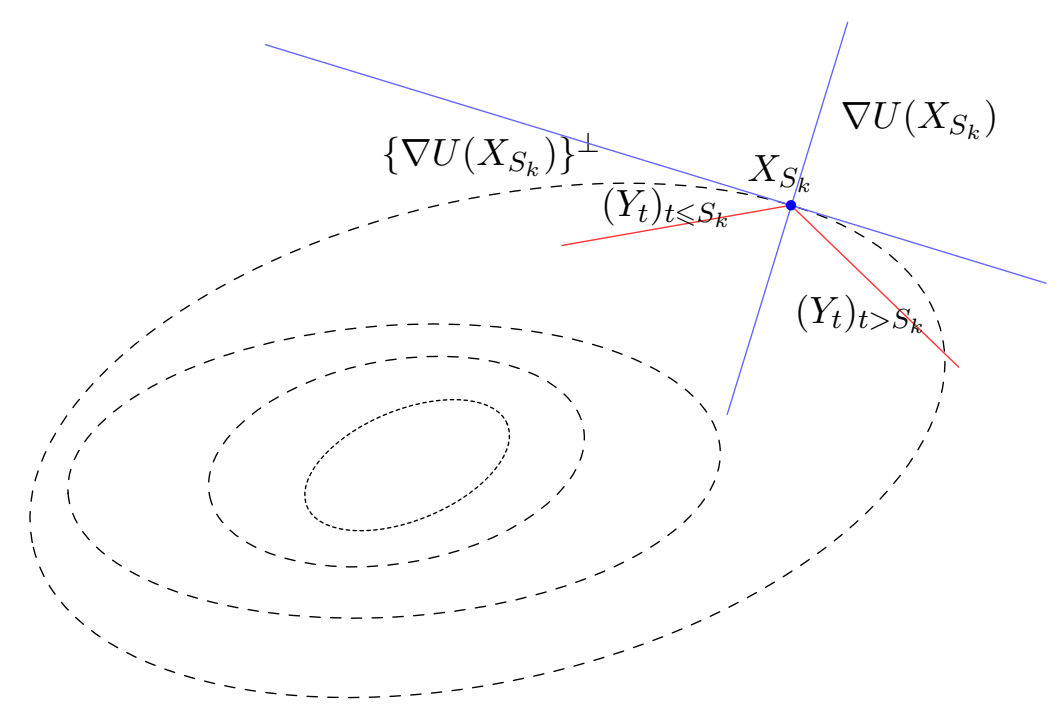

FigURE 1. Representation of a deterministic bounce in the case $g=\nabla U$ for some potential $U$. The dashed lines are level sets of $U$. At the jump time $S_{k}, V_{S_{k}}$ is reflected with respect to $\nabla U\left(X_{S_{k}}\right)$.

distribution $\mu_{\mathrm{v}}$ is the $\mathrm{PDMP}$ on $\mathrm{M}=\mathbb{R}^{d} \times \mathrm{V}$ with characteristics $\left(\varphi,\left(\lambda_{i}, Q_{i}\right)_{i \in \llbracket 1,2 \rrbracket}\right)$ where $\varphi$ is given by (4) and for all $(x, y) \in \mathbb{R}^{d} \times \mathrm{V}, \mathrm{A} \in \mathcal{B}\left(\mathbb{R}^{d}\right), \lambda_{1}(x, y)=\langle y, \nabla U(x)\rangle_{+}$, $\lambda_{2}(x, y)=\lambda_{\mathrm{c}}, Q_{1}((x, y), \mathrm{A} \times\{\mathrm{R}(x, y)\})=\delta_{x}(\mathrm{~A})$ and $Q_{2}((x, y), \cdot)=\delta_{x} \otimes \mu_{\mathrm{v}}$, where $\mathrm{R}$ is given by (5) with $g(x)=\nabla U(x)$. Note that $\left(\lambda_{1}, Q_{1}\right)$ is the pure bounce mechanism associated with $g$ (see Figure 1 ) and $\left(\lambda_{2}, Q_{2}\right)$ is a refreshment mechanism.

Variants of the BPS with randomized bounces have been recently introduced in [20, $25,23]$.

\section{Alternative constructions}

Consider PDMP characteristics $\left(\varphi,\left(\lambda_{i}, Q_{i}\right)_{i \in \llbracket 1, \ell \rrbracket}\right)$, an initial distribution $\mu_{0} \in \mathcal{P}(\mathrm{M})$ and the associated process $\left(X_{t}\right)_{t \geqslant 0}$ defined in Section 2. The goal of this Section is to construct another process $\left(Y_{t}\right)_{t \geqslant 0}$ on the same probability space $(\Omega, \mathcal{F}, \mathbb{P})$ with the same distribution on $\mathrm{D}\left(\mathbb{R}_{+}, \mathrm{M} \cup\{\infty\}\right)$ as $\left(X_{t}\right)_{t \geqslant 0}$. This alternative construction will turn out to be interesting to obtain a characterization of the distribution of the first jump time of type $i \in\{1, \ldots, \ell\}$; see Proposition 4 below. The main difference between the two constructions is the following. In Construction 1, the sequence $\left(E_{j, k+1}\right)_{j \in \llbracket 1, \ell \rrbracket}$ is only used after the $k^{\text {th }}$ jump to define $S_{k+1}$, and they are all discarded afterwards. In contrast, Construction 2 takes advantage of the memoryless property of the exponential distribution: the random variables $\left(\tilde{H}_{j, k+1}\right)_{j \in \llbracket 1, \ell \rrbracket}$ which play the same role than $\left(E_{j, k+1}\right)_{j \in \llbracket 1, \ell \rrbracket}$ to define the jump times $S_{k+1}$ are simply updated so that they are i.i.d. exponential random variables independent of $\mathcal{F}_{k}^{\prime}$ (see Lemma 3), which is the main reason why Construction 2 defined a process with the same distribution than Construction 1 (see Proposition 2). 
Construction 2. Let $W_{0}$ be a random variable with distribution $\mu_{0} \in \mathcal{P}(\mathrm{M})$ and $\left(E_{j, k}, U_{j, k}\right)_{j \in \llbracket 1, \ell \rrbracket, k \in \mathbb{N}^{*}}$ be an i.i.d. family, independent of $W_{0}$, such that for all $k \in \mathbb{N}$ and $j \in \llbracket 1, \ell \rrbracket, U_{j, k}$ is uniformly distributed on $[0,1]$ and $E_{j, k}$ is an exponential random variable with parameter 1 , independent of $U_{j, k}$.

Set $\tilde{S}_{0}=0, Y_{0}^{\prime}=W_{0}, \tilde{I}_{0}=0, \bar{N}_{0}=1, \tilde{H}_{j, 1}=E_{j, 1}$ and $\tilde{N}_{j, 1}=1$, for $j \in \llbracket 1, \ell \rrbracket$. Suppose that $\left(Y_{t}\right)_{t \leqslant S_{k}}$ and $\left(Y_{k}^{\prime}, \tilde{S}_{k}, \tilde{I}_{k}, \bar{N}_{k},\left(\tilde{H}_{j, k+1}, \tilde{N}_{j, k+1}\right)_{j \in \llbracket 1, \ell \rrbracket}\right)$ have been defined for some $k \in \mathbb{N}$. Set

$$
\tilde{S}_{j, k+1}=\inf \left\{t>\tilde{S}_{k}: \tilde{H}_{j, k+1}<\int_{\tilde{S}_{k}}^{t} \lambda_{j}\left(s, \varphi_{\tilde{S}_{k}, s}\left(Y_{k}^{\prime}\right)\right) \mathrm{d} s\right\}, \tilde{S}_{k+1}=\min _{j \in \llbracket 1, \ell \rrbracket} \tilde{S}_{j, k+1} .
$$

- If $\tilde{S}_{k+1}=+\infty$, set $\tilde{S}_{m}=+\infty, Y_{m}^{\prime}=\infty, \tilde{I}_{m}=1$ for all $m>k$ and $Y_{t}=\varphi_{\tilde{S}_{k}, t}\left(Y_{k}^{\prime}\right)$ for $t \geqslant \tilde{S}_{k}$.

- If $\tilde{S}_{k+1}<+\infty$, set

$$
\begin{aligned}
& \tilde{I}_{k+1}=\min \left\{j \in \llbracket 1, \ell \rrbracket, \tilde{S}_{j, k+1}=\tilde{S}_{k+1}\right\} \quad, \quad \bar{N}_{k+1}=\tilde{N}_{\tilde{I}_{k+1}, k+2}=\tilde{N}_{\tilde{I}_{k+1}, k+1}+1 \text {, } \\
& Y_{k+1}^{\prime}=\mathbf{G}_{\tilde{I}_{k+1}}\left(\tilde{S}_{k+1}, \varphi_{\tilde{S}_{k}, \tilde{S}_{k+1}}\left(Y_{k}^{\prime}\right), U_{\tilde{I}_{k+1}, \bar{N}_{k+1}}\right), \quad \tilde{H}_{\tilde{I}_{k+1}, k+2}=E_{\tilde{I}_{k+1}, \bar{N}_{k+1}},
\end{aligned}
$$

and for $j \neq \tilde{I}_{k+1}$,

$$
\tilde{H}_{j, k+2}=\tilde{H}_{j, k+1}-\int_{\tilde{S}_{k}}^{\tilde{S}_{k+1}} \lambda_{j}\left(s, \varphi_{\tilde{S}_{k}, s}\left(Y_{k}^{\prime}\right)\right) \mathrm{d} s, \quad \tilde{N}_{j, k+2}=\tilde{N}_{j, k+1} .
$$

Set $Y_{t}=\varphi_{\tilde{S}_{k}, t}\left(Y_{k}^{\prime}\right)$ for $t \in\left(\tilde{S}_{k}, \tilde{S}_{k+1}\right)$ and $Y_{\tilde{S}_{k+1}}=Y_{k+1}^{\prime}$.

For $t \geqslant \sup _{k \in \mathbb{N}} \tilde{S}_{k}$, set $Y_{t}=\infty$.

We show in the following result that the two constructions we consider define the same distribution on $\mathrm{D}\left(\mathbb{R}_{+}, \mathrm{M} \cup\{\infty\}\right)$.

Proposition 2. The two Markov chains $\left(X_{k}^{\prime}, S_{k}, I_{k}\right)_{k \in \mathbb{N}}$ and $\left(Y_{k}^{\prime}, \tilde{S}_{k}, \tilde{I}_{k}\right)_{k \in \mathbb{N}}$ have the same distribution on $\left((\mathrm{M} \cup\{\infty\}) \times\left(\mathbb{R}_{+} \cup\{+\infty\}\right)\right)^{\mathbb{N}}$. Therefore, $\left(X_{t}\right)_{t \geqslant 0}$ and $\left(Y_{t}\right)_{t \geqslant 0}$ have the same distribution on $\mathrm{D}\left(\mathbb{R}_{+}, \mathrm{M} \cup\{\infty\}\right)$.

We preface the proof by a lemma. Denote by $\left(\tilde{\mathcal{F}}_{k}\right)_{k \in \mathbb{N}}$ and $\left(\tilde{\mathcal{F}}_{k}^{\prime}\right)_{k \in \mathbb{N}}$ the filtration associated with the sequence of random variables $\left(Y_{k}^{\prime}, \tilde{S}_{k}, \tilde{I}_{k}\right)_{k \in \mathbb{N}}$ and $\left(Y_{k}^{\prime}, \tilde{S}_{k}\right)_{k \in \mathbb{N}}$.

Lemma 3. Let $k \in \mathbb{N}$. For all $i \in \llbracket 1, \ell \rrbracket$, given $\left\{\tilde{I}_{k}=i\right\} \cap\left\{\tilde{S}_{k}<+\infty\right\},\left(\tilde{H}_{j, k+1}\right)_{j \in \llbracket 1, \ell \rrbracket \backslash\{i\}}$ are i.i.d. exponential random variables with parameter 1 , independent of $\tilde{\mathcal{F}}_{k}^{\prime}$. In addition, given $\left\{\tilde{S}_{k}<+\infty\right\},\left(\tilde{H}_{j, k+1}\right)_{j \in \llbracket 1, \ell \rrbracket}$ are i.i.d. exponential random variables with parameter 1 , independent of $\tilde{\mathcal{F}}_{k}^{\prime}$. Finally, $\mathbb{P}\left(\tilde{S}_{j, k+1}=\tilde{S}_{j^{\prime}, k+1}<+\infty \mid \tilde{\mathcal{F}}_{k}^{\prime}\right)=0$ for any $j, j^{\prime} \in \llbracket 1, \ell \rrbracket$.

Proof. First, it is sufficient to consider the case $\ell>1$, since in the case $\ell=1, H_{1, k+1}=$ $\bar{E}_{1, k+1}$ for any $k \in \mathbb{N}$. 
Note that the first statement is equivalent to for any $i \in \llbracket 1, \ell \rrbracket,\left(t_{j}\right)_{j \in \llbracket 1, \ell \rrbracket \backslash\{i\}} \in \mathbb{R}_{+}^{\ell-1}$, $\mathrm{B} \in \tilde{\mathcal{F}}_{k}^{\prime}$, setting $\mathrm{A}_{i, k}=\bigcap_{j=1, j \neq i}^{\ell}\left\{\tilde{H}_{j, k+1} \geqslant t_{j}\right\}$,

$$
\mathbb{E}\left[\mathbb{1}_{\mathbb{R}_{+}}\left(\tilde{S}_{k}\right) \mathbb{1}_{i}\left(\tilde{I}_{k}\right) \mathbb{1}_{\mathrm{A}_{k, i}} \mathbb{1}_{\mathrm{B}}\right]=\exp \left(-\sum_{j \in \llbracket 1, \ell \rrbracket \backslash\{i\}} t_{j}\right) \mathbb{E}\left[\mathbb{1}_{\mathbb{R}_{+}}\left(\tilde{S}_{k}\right) \mathbb{1}_{i}\left(\tilde{I}_{k}\right) \mathbb{1}_{\mathrm{B}}\right] .
$$

Indeed taking $\mathrm{B}=\Omega$, we get $\mathbb{P}\left(\mathrm{A}_{k, i} \mid \tilde{I}_{k}=i, \tilde{S}_{k}<+\infty\right)=\exp \left(-\sum_{j \in \llbracket 1, \ell \rrbracket} t_{j}\right)$. This statement is also equivalent to

(6)

$$
\mathbb{1}_{\mathbb{R}_{+}}\left(\tilde{S}_{k}\right) \mathbb{P}\left(\bigcap_{j=1, j \neq i}^{\ell}\left\{\tilde{H}_{j, k+1} \geqslant t_{j}\right\} \cap\left\{\tilde{I}_{k}=i\right\} \mid \tilde{\mathcal{F}}_{k}^{\prime}\right)=\exp \left(-\sum_{j \in \llbracket 1, \ell \rrbracket \backslash\{i\}} t_{j}\right) \mathbb{1}_{\mathbb{R}_{+}}\left(\tilde{S}_{k}\right) \mathbb{P}\left(\tilde{I}_{k}=i \mid \tilde{\mathcal{F}}_{k}^{\prime}\right),
$$

which is statement that we show. Similarly, we show that for the second statement, it is sufficient to show that $\mathbb{1}_{\mathbb{R}_{+}}\left(\tilde{S}_{k}\right) \mathbb{P}\left(\bigcap_{j=1}^{\ell}\left\{\tilde{H}_{j, k+1} \geqslant t_{j}\right\} \mid \tilde{\mathcal{F}}_{k}^{\prime}\right)=\exp \left(-\sum_{j \in \llbracket 1, \ell \rrbracket} t_{j}\right)$ for any $\left(t_{j}\right)_{j \in \llbracket 1, \ell \rrbracket} \in \mathbb{R}_{+}^{\ell}$.

The proof is by induction on $k \in \mathbb{N}^{*}$. For $k=0$, by definition the first two statements hold. The last one follows from (1) for $k=0$ and Construction 1, Construction 2.

Assume now that the result holds for $k \in \mathbb{N}$ and let $i \in \llbracket 1, \ell \rrbracket$.

First, we show that the last statement for $k+1$ : for any $j^{\prime}, j \in \llbracket 1, \ell \rrbracket, \mathbb{P}\left(S_{j, k+1}=\right.$ $\left.S_{j^{\prime}, k+1}<+\infty \mid \tilde{\mathcal{F}}_{k}^{\prime}\right)=0$. Indeed, given $\tilde{\mathcal{F}}_{k}^{\prime}$, by definition $S_{j, k+1}=S_{j^{\prime}, k+1}$ if and only if $\psi_{j, k+1}^{\overleftarrow{\leftarrow}}\left(H_{j, k+1}\right)=\psi_{j^{\prime}, k+1}^{\overleftarrow{n^{\prime}}}\left(H_{j^{\prime}, k+1}\right)$, where $\psi_{j, k+1}^{\overleftarrow{j_{j}}}$ is the generalized inverse of the nondecreasing function $\psi_{j, k+1}(t)=\int_{\tilde{S}_{k}}^{\tilde{S}_{k}+t} \lambda_{j}\left(s, \varphi_{\tilde{S}_{k}, s}\left(Y_{k}^{\prime}\right)\right) \mathrm{d} s$. Since $\psi_{j^{\prime}, k+1}$ is continuous, we get using properties of the generalized inverse that given $\tilde{\mathcal{F}}_{k}^{\prime}, S_{j, k+1}=S_{j^{\prime}, k+1}$ if and only if $\psi_{j^{\prime}, k+1} \circ \psi_{j, k+1}^{\overleftarrow{ }}\left(H_{j, k+1}\right)=H_{j^{\prime}, k+1}$, which implies by the induction hypothesis, $\mathbb{P}\left(S_{j, k+1}=S_{j^{\prime}, k+1} \mid \tilde{\mathcal{F}}_{k}^{\prime}\right)=0$. It follows that given $\tilde{\mathcal{F}}_{k+1}^{\prime}$ and $\left\{\tilde{S}_{k+1}<+\infty\right\}$, using again properties of the generalized inverse,

$$
\begin{aligned}
\left\{I_{k+1}=i\right\}= & \left\{H_{i, k+1}=\psi_{i, k+1}\left(\tilde{S}_{i, k+1}\right)\right\} \cap\left\{\cap_{j \in \llbracket 1, \ell \rrbracket \backslash\{i\}}\left\{\tilde{S}_{j, k+1}>\tilde{S}_{i, k+1}\right\}\right\} \\
& =\left\{H_{i, k+1}=\psi_{i, k+1}\left(\tilde{S}_{k+1}-\tilde{S}_{k}\right)\right\} \cap\left\{\cap_{j \in \llbracket 1, \ell \rrbracket \backslash\{i\}}\left\{H_{j, k+1}>B_{j, k+1}\right\}\right\},
\end{aligned}
$$

where we have set $B_{j, k+1}=\mathbb{1}_{\mathbb{R}_{+}}\left(\tilde{S}_{k+1}\right) \int_{\tilde{S}_{k}}^{\tilde{S}_{k+1}}\left\{\lambda_{j}\left(s, \varphi_{\tilde{S}_{k}, s}\left(Y_{k}^{\prime}\right)\right)\right\} \mathrm{d} s$.

We are now able to show that the first two statements hold for $k+1$. Note that almost surely, we have by (7) and the induction hypothesis, for all $t_{1}, \ldots, t_{\ell} \geqslant 0$,

$$
\begin{aligned}
& \mathbb{1}_{\mathbb{R}_{+}}\left(\tilde{S}_{k+1}\right) \mathbb{P}\left(\bigcap_{j=1, j \neq i}^{\ell}\left\{\tilde{H}_{j, k+2} \geqslant t_{j}\right\} \cap\left\{\tilde{I}_{k+1}=i\right\} \mid \tilde{\mathcal{F}}_{k+1}^{\prime}\right) \\
& =\mathbb{1}_{\mathbb{R}_{+}}\left(\tilde{S}_{k+1}\right) \mathbb{P}\left(\left\{H_{i, k+1}=\psi_{i, k+1}\left(\tilde{S}_{k+1}\right)\right\} \cap \bigcap_{j=1, j \neq i}^{\ell}\left\{\tilde{H}_{j, k+1}-B_{j, k+1} \geqslant t_{j}\right\} \cap\left\{H_{j, k+1}>B_{j, k+1}\right\} \mid \tilde{\mathcal{F}}_{k+1}^{\prime}\right) \\
& =\mathbb{1}_{\mathbb{R}_{+}}\left(\tilde{S}_{k+1}\right) \exp \left(-\sum_{j=1, j \neq i}^{\ell}\left\{t_{j}+B_{j, k+1}\right\}\right) \mathbb{P}\left(\left\{H_{i, k+1}=\psi_{i, k+1}\left(\tilde{S}_{k+1}\right)\right\} \mid \tilde{\mathcal{F}}_{k+1}^{\prime}\right)
\end{aligned}
$$

establishing (6) for $k+1$. For the second statement, using (6) for $k+1$ and that $E_{i, \bar{N}_{k+2}}$ is an exponential random variable independent of $\sigma\left(\tilde{\mathcal{F}}_{k+1}^{\prime},\left(\tilde{H}_{j, k+1}\right)_{j \in \llbracket 1, \ell \rrbracket}\right)$ given $\left\{I_{k+1}=i\right\}$ for all $i \in \llbracket 1, \ell \rrbracket$, we obtain that

$$
\begin{aligned}
\mathbb{1}_{\mathbb{R}_{+}}\left(\tilde{S}_{k+1}\right) \mathbb{P}\left(\bigcap_{j=1}^{\ell}\left\{\tilde{H}_{j, k+2} \geqslant t_{j}\right\} \mid \tilde{\mathcal{F}}_{k+1}^{\prime}\right) \\
\quad=\mathbb{1}_{\mathbb{R}_{+}}\left(\tilde{S}_{k+1}\right) \sum_{i=1}^{\ell} \mathbb{P}\left(\bigcap_{j=1}^{\ell}\left\{\tilde{H}_{j, k+2} \geqslant t_{j}\right\} \cap\left\{I_{k+1}=i\right\} \mid \tilde{\mathcal{F}}_{k+1}^{\prime}\right)
\end{aligned}
$$




$$
\begin{aligned}
& =\mathbb{1}_{\mathbb{R}_{+}}\left(\tilde{S}_{k+1}\right) \sum_{i=1}^{\ell} \mathbb{P}\left(\bigcap_{j=1, j \neq i}^{\ell}\left\{\tilde{H}_{j, k+2} \geqslant t_{j}\right\} \cap\left\{I_{k+1}=i\right\} \cap\left\{\bar{E}_{i, \bar{N}_{k+2}} \geqslant t_{i}\right\} \mid \tilde{\mathcal{F}}_{k+1}^{\prime}\right) \\
& =\mathbb{1}_{\mathbb{R}_{+}}\left(\tilde{S}_{k+1}\right) \exp \left(-\sum_{j=1}^{\ell} t_{j}\right) \sum_{i=1}^{\ell} \mathbb{P}\left(I_{k+1}=i \mid \tilde{\mathcal{F}}_{k+1}^{\prime}\right)=\mathbb{1}_{\mathbb{R}_{+}}\left(\tilde{S}_{k+1}\right) \exp \left(-\sum_{j=1}^{\ell} t_{j}\right) .
\end{aligned}
$$

Proof of Proposition 2. We show that the two processes $\left(X_{k}^{\prime}, S_{k}, I_{k}\right)_{k \in \mathbb{N}}$ and $\left(Y_{k}^{\prime}, \tilde{S}_{k}, \tilde{I}_{k}\right)_{k \in \mathbb{N}}$ have the same distribution. Note that, since $\left(X_{0}^{\prime}, S_{0}, I_{0}\right)$ and $\left(Y_{0}^{\prime}, \tilde{S}_{0}, \tilde{I}_{0}\right)$ have the same distribution, this result is equivalent to show that $\left(Y_{k}^{\prime}, \tilde{S}_{k}, \tilde{I}_{k}\right)_{k \in \mathbb{N}}$ is also a Markov chain with a Markov kernel characterized by (3). Let $k \in \mathbb{N}, \mathrm{A} \in \mathcal{B}(\mathrm{M}), t \geqslant \tilde{S}_{k}, i \in \llbracket 1, \ell \rrbracket$. Note first that given $\left\{I_{k+1}=i\right\}$ and $\tilde{S}_{k}$, for $i \in \llbracket 1, \ell \rrbracket, \psi_{i, k+1}^{\overleftarrow{H}}\left(\tilde{H}_{i, k+1}\right)=\tilde{S}_{i, k+1}-\tilde{S}_{k}$, where $\psi_{i, k+1}^{\overleftarrow{ }}$ is generalized inverse of $\psi_{i, k+1}(t)=\int_{\tilde{S}_{k}}^{\tilde{S}_{k}+t} \lambda_{i}\left(s, \varphi_{\tilde{S}_{k}, s}\left(Y_{k}^{\prime}\right)\right) \mathrm{d} s$. Then, By Lemma 3 and definition of $\left(Y_{\tilde{k}}^{\prime}, \tilde{S}_{\tilde{k}}, \tilde{I}_{\tilde{k}}\right)_{\tilde{k} \in \mathbb{N}}$, since, given $\left\{\tilde{I}_{k+1}=i\right\} \cap\left\{\tilde{S}_{k+1}<+\infty\right\}, Y_{k+1}^{\prime}=$ $\mathbf{G}\left(\tilde{S}_{i, k+1}, \varphi_{\tilde{S}_{k}, \tilde{S}_{i, k+1}}\left(Y_{k}^{\prime}\right), U_{i, \bar{N}_{k+1}}\right)$ and $U_{i, \bar{N}_{k+1}}$ is independent of $\sigma\left(\tilde{\mathcal{F}}_{k},\left(\tilde{H}_{j, k+1}\right)_{j \in \llbracket 1, \ell \rrbracket}\right)$, then $\operatorname{setting} \mathcal{G}_{i, k+1}=\sigma\left(\tilde{\mathcal{F}}_{k}, \tilde{H}_{i, k+1}, U_{i, \bar{N}_{k+1}}\right)$ and $B_{j, k+1}=\mathbb{1}_{\mathbb{R}_{+}}\left(\tilde{S}_{i, k+1}\right) \int_{\tilde{S}_{k}}^{\tilde{S}_{i, k+1}} \lambda_{j}\left(s, \varphi_{\tilde{S}_{k}, s}\left(Y_{k}^{\prime}\right)\right) \mathrm{d} s$, for $j \in \llbracket 1, \ell \rrbracket \backslash\{i\}$, we have

$$
\begin{aligned}
& \mathbb{P}\left(Y_{k+1}^{\prime} \in \mathrm{A}, \tilde{S}_{k+1} \leqslant t, \tilde{I}_{k+1}=i \mid \tilde{\mathcal{F}}_{k}\right) \\
& =\mathbb{P}\left(Y_{k+1}^{\prime} \in \mathrm{A}, \tilde{S}_{i, k+1} \leqslant t, \tilde{S}_{i, k+1}<\tilde{S}_{j, k+1}, \text { for all } j \in \llbracket 1, \ell \rrbracket \backslash\{i\}, \tilde{I}_{k+1}=i \mid \tilde{\mathcal{F}}_{k}\right) \\
& =\mathbb{E}\left[\mathbb{1}_{\mathrm{A}}\left(Y_{k+1}^{\prime}\right) \mathbb{1}_{\left[\tilde{S}_{k}, t\right]}\left(\tilde{S}_{i, k+1}\right) \mathbb{P}\left(\bigcap_{j \in \llbracket 1, \ell \rrbracket, j \neq i}\left\{\tilde{S}_{i, k+1}<\tilde{S}_{j, k+1}\right\} \mid \mathcal{G}_{i, k+1}\right) \mid \tilde{\mathcal{F}}_{k}\right] \\
& =\mathbb{E}\left[\mathbb{1}_{\mathrm{A}}\left(Y_{k+1}^{\prime}\right) \mathbb{1}_{\left[\tilde{S}_{k}, t\right]}\left(\tilde{S}_{i, k+1}\right) \mathbb{P}\left(\bigcap_{j \in \llbracket 1, \ell \rrbracket, j \neq i}\left\{B_{j, k+1}<\tilde{H}_{j, k+1}\right\} \mid \mathcal{G}_{i, k+1}\right) \mid \tilde{\mathcal{F}}_{k}\right] \\
& =\mathbb{E}\left[\mathbb{1}_{\mathrm{A}}\left(Y_{k+1}^{\prime}\right) \mathbb{1}_{\left[\tilde{S}_{k}, t\right]}\left(\tilde{S}_{i, k+1}\right) \exp \left\{-\sum_{j=1, j \neq i}^{\ell} \int_{\tilde{S}_{k}}^{\tilde{S}_{i, k+1}} \lambda_{j}\left(u, \varphi_{\tilde{S}_{k}, u}\left(Y_{k}^{\prime}\right)\right) \mathrm{d} u\right\} \mid \tilde{\mathcal{F}}_{k}\right] .
\end{aligned}
$$

The proof then follows from $\psi_{i, k+1}^{\overleftarrow{H}}\left(\tilde{H}_{i, k+1}\right)=\tilde{S}_{i, k+1}-\tilde{S}_{k}$ Lemma 3 and the definition of $Y_{k+1}^{\prime}$.

For $k \geqslant 1$ with $S_{k}<\infty$, we say that, at time $S_{k}$, the process $\left(X_{t}\right)_{t \geqslant 0}$ given by Construction 1 has made a jump of type $I_{k}$, or equivalently that $S_{k}$ is a jump time of type $I_{k}$. Define

$$
T^{(j)}=\inf \left\{S_{k}: k \geqslant 1, I_{k}=j\right\},
$$

the first jump time of type $j$. Then, one example of application of Proposition 2 is the following result.

Proposition 4. Let $\ell=\ell_{1}+\ell_{2}$ with $\ell_{1}, \ell_{2} \in \mathbb{N}^{*}$. Let $\left(X_{t}\right)_{t \geqslant 0}$ be a PDMP on $\mathrm{M}$ with characteristics $\left(\varphi,\left(\lambda_{i}, Q_{i}\right)_{i \in \llbracket 1, \ell \rrbracket}\right)$ given by Construction 1 and initial distribution $\mu_{0}$. Define $T=\min \left\{T^{(i)}: i \in \llbracket \ell_{1}+1, \ell \rrbracket\right\}$, where $T^{(j)}$ is given by (8) for all $j \in \llbracket 1, \ell \rrbracket$. Then the cumulative distribution function of $T$ is given for all $u \geqslant 0$ by

$$
\mathbb{P}(T \leqslant u)=\mathbb{P}\left(E<\sum_{i \in \llbracket \ell_{1}+1 \rrbracket}^{\ell} \int_{0}^{u \wedge \tau_{\infty}(Z)} \lambda_{i}\left(s, Z_{s}\right) \mathrm{d} s\right),
$$

where $\left(Z_{t}\right)_{t \geqslant 0}$ is a PDMP with characteristics $\left(\varphi,\left(\lambda_{i}, Q_{i}\right)_{i \in \llbracket 1, \ell_{1} \rrbracket}\right)$ and initial distribution $\mu_{0}$, and $E$ is a standard exponential random variable independent of $\left(Z_{t}\right)_{t \geqslant 0}$. 
Proof. Let $\left(Y_{t}\right)_{t \geqslant 0}$ be a PDMP defined by Construction 2 with characteristics $\left(\varphi,\left(\lambda_{i}\right.\right.$, $\left.\left.Q_{i}\right)_{i \in \llbracket 1, \ell \rrbracket}\right)$ and initial distribution $\mu_{0}$. Define similarly to $\left(X_{t}\right)_{t \geqslant 0}$ for all $j \in \llbracket 1, \ell \rrbracket$, $\tilde{T}^{(j)}=\inf \left\{\tilde{S}_{k}: k \geqslant 1, \tilde{I}_{k}=j\right\}$ and $\tilde{T}=\min \left\{\tilde{T}^{(i)}: i \in \llbracket \ell_{1}+1, \ell_{2} \rrbracket\right\}$. Note that since by Proposition $2,\left(X_{k}^{\prime}, S_{k}, I_{k}\right)_{k \in \mathbb{N}}$ and $\left(Y_{k}^{\prime}, \tilde{S}_{k}, \tilde{I}_{k}\right)_{k \in \mathbb{N}}$ has the same distribution, $T$ and $\tilde{T}$ have the same distribution and it suffices to show that the cumulative distribution function of $\tilde{T}$ is given by (9).

Let $\left(Z_{t}\right)_{t \geqslant 0}$ be a PDMP with characteristics $\left(\varphi,\left(\lambda_{i}, Q_{i}\right)_{i \in \llbracket 1, \ell_{1} \rrbracket}\right)$ and initial distribution $\mu_{0}$ defined by Construction 2 and based on the random variables $W_{0},\left(E_{j, k}, U_{j, k}\right)_{j \in \llbracket 1, \ell_{1} \rrbracket, k \in \mathbb{N}}$, and let $\left(Z_{k}^{\prime}, R_{k}, J_{k}\right)_{k \in \mathbb{N}}$ be the corresponding embedded chain. By construction, for all $t \leqslant \tilde{T} \wedge \tau_{\infty}(Z), Y_{t}=Z_{t}$. In addition, define

$$
N=\inf \left\{k \in \mathbb{N}^{*}: \text { there exists } i \in \llbracket \ell_{1}+1, \ell \rrbracket, \tilde{I}_{k}=i\right\} .
$$

By definition, $\tilde{T}=\tilde{S}_{N}$ on $\{\tilde{T}<+\infty\}$ and for all $k \in \mathbb{N}^{*}$, on $\{N=k\}$ for all $t \in\left[0, \tilde{S}_{k}\right]$, $Y_{t}=Z_{t}$, and for all $n \leqslant k, Y_{n}^{\prime}=Z_{n}^{\prime}, \tilde{S}_{n}=R_{n}$. Therefore, for all $k \in \mathbb{N}^{*}$, on $\{N \geqslant$ $k\} \cap\left\{\tau_{\infty}(Z) \geqslant t\right\}$, for all $i \in \llbracket \ell_{1}+1, \ell \rrbracket$, we have by induction

$$
\tilde{S}_{i, k}=\inf \left\{t>R_{k-1}: \tilde{H}_{i, k}<\int_{R_{k-1}}^{t} \lambda_{i}\left(s, \varphi_{R_{k-1}, s}\left(Z_{k}^{\prime}\right)\right) \mathrm{d} s\right\}=\inf \left\{t>0: E_{i, 0}<\int_{0}^{t} \lambda_{i}\left(s, Z_{s}\right) \mathrm{d} s\right\} .
$$

Since $\left\{\tilde{T}<\tau_{\infty}(Z)\right\} \subset\{\tilde{T}<+\infty\}$, we thus obtain

$$
\begin{aligned}
\left\{\tilde{T}<\tau_{\infty}(Z)\right\} \cap\{\tilde{T} \geqslant t\} & =\left\{\tilde{T}<\tau_{\infty}(Z)\right\} \cap\left\{\bigcap_{k \in \mathbb{N}^{*}, i \in \llbracket \ell_{1}+1, \ell \rrbracket}\left\{\tilde{S}_{i, k} \geqslant t\right\} \cap\{N=k\}\right\} \\
& =\left\{\tilde{T}<\tau_{\infty}(Z)\right\} \cap\left\{\bigcap_{i \in \llbracket \ell_{1}+1, \ell \rrbracket}\left\{E_{i, 0} \geqslant \int_{0}^{t} \lambda_{i}\left(s, Z_{s}\right) \mathrm{d} s\right\}\right\} .
\end{aligned}
$$

In addition, since $\{\tilde{T}=+\infty\}=\left\{\tilde{T} \geqslant \tau_{\infty}(Z)\right\}$, we have

$$
\begin{aligned}
\left\{\tilde{T} \geqslant \tau_{\infty}(Z) \vee t\right\}=\{\tilde{T}=+\infty\} & =\bigcap_{i \in \llbracket \ell_{1}+1, \ell \rrbracket, k \in \mathbb{N}^{*}}\left\{\tilde{S}_{i, k} \geqslant R_{k}\right\} \\
& =\bigcap_{i \in \llbracket \ell_{1}+1, \ell \rrbracket}\left\{E_{i, 0} \geqslant \int_{0}^{\tau_{\infty}(Z)} \lambda_{i}\left(s, Z_{s}\right) \mathrm{d} s\right\} .
\end{aligned}
$$

Combining (10) and (11) and since $\left(E_{i, 0}\right)_{i \in \llbracket \ell_{1}+1, \ell_{2} \rrbracket}$ is independent of $\left(Z_{s}\right)_{s \geqslant 0}$, we get

$\mathbb{P}(\tilde{T} \geqslant t)=\mathbb{P}\left(\bigcap_{i \in \llbracket \ell_{1}+1, \ell \rrbracket}\left\{E_{i, 0} \geqslant \int_{0}^{\tau_{\infty}(Z) \wedge t} \lambda_{i}\left(s, Z_{s}\right) \mathrm{d} s\right\}\right)=\mathbb{E}\left[\exp \left(-\sum_{i=\ell_{1}+1}^{\ell} \int_{0}^{\tau_{\infty}(Z) \wedge t} \lambda_{i}\left(s, Z_{s}\right) \mathrm{d} s\right)\right]$

which concludes the proof. 


\section{Superposition and Splitting of JUmp mechanisms}

We now introduce a tool to deal with PDMP: superposition and splitting of jump mechanisms. This is a natural generalization of the fact that the sum of two independent Poisson processes with jump rates $\bar{\lambda}_{1}, \bar{\lambda}_{2}: \mathbb{R}_{+} \rightarrow \mathbb{R}_{+}$, is a Poisson process associated with the jump rate $\bar{\lambda}_{1}+\bar{\lambda}_{2}$.

Theorem 5. Let $\left(\lambda_{i, 1}, Q_{i, 1}\right)_{i \in \llbracket 1, \ell_{1} \rrbracket}$ and $\left(\lambda_{i, 2}, Q_{i, 2}\right)_{i \in \llbracket 1, \ell_{2} \rrbracket}$ be two families of jump mechanisms on $\mathrm{M}$. Suppose that for all $(t, x, \mathrm{~A}) \in \mathbb{R}_{+} \times \mathrm{M} \times \mathcal{B}(\mathrm{M})$,

$$
\sum_{i=1}^{\ell_{1}} \lambda_{i, 1}(t, x)\left(Q_{i, 1}(t, x, \mathrm{~A})-\delta_{x}(\mathrm{~A})\right)=\sum_{i=1}^{\ell_{2}} \lambda_{i, 2}(t, x)\left(Q_{i, 2}(t, x, \mathrm{~A})-\delta_{x}(\mathrm{~A})\right) .
$$

Then, for all differential flow $\varphi$ and initial distribution $\mu_{0} \in \mathcal{P}(\mathrm{M})$,

$$
\operatorname{PDMP}\left(\varphi,\left(\lambda_{i, 1}, Q_{i, 1}\right)_{i \in \llbracket 1, \ell_{1} \rrbracket}, \mu_{0}\right)=\operatorname{PDMP}\left(\varphi,\left(\lambda_{i, 2}, Q_{i, 2}\right)_{i \in \llbracket 1, \ell_{2} \rrbracket}, \mu_{0}\right) .
$$

If $\left(\lambda_{i}, Q_{i}\right)_{i \in \llbracket 1, \ell \rrbracket}$ is a family of jump mechanisms, we define the associated minimal jump mechanism $\left(\lambda_{\mathrm{m}}, Q_{\mathrm{m}}\right)$ and the associated total jump mechanism $\left(\lambda_{\mathrm{T}}, Q_{\mathrm{T}}\right)$ for all $(t, x, \mathrm{~A}) \in \mathbb{R}_{+} \times \mathrm{M} \times \mathcal{B}(\mathrm{M})$, by

$$
\begin{aligned}
\lambda_{\mathrm{m}}(t, x) & =\sum_{i=1}^{\ell} \lambda_{i}(t, x) Q_{i}(t, x, \mathrm{M} \backslash\{x\}) \\
Q_{\mathrm{m}}(t, x, \mathrm{~A}) & = \begin{cases}\lambda_{\mathrm{m}}^{-1}(t, x) \sum_{i=1}^{\ell} \lambda_{i}(t, x) Q_{i}(t, x, \mathrm{~A} \backslash\{x\}) & \text { if } \lambda_{\mathrm{m}}(t, x) \neq 0, \\
\delta_{x}(\mathrm{~A}) & \text { otherwise. }\end{cases} \\
\lambda_{\mathrm{T}}(t, x) & =\sum_{i=1}^{\ell} \lambda_{i}(t, x) \\
Q_{\mathrm{T}}(t, x, \mathrm{~A}) & = \begin{cases}\lambda_{\mathrm{T}}^{-1}(t, x) \sum_{i=1}^{\ell} \lambda_{i}(t, x) Q_{i}(t, x, \mathrm{~A}) & \text { if } \lambda_{\mathrm{T}}(t, x) \neq 0, \\
\delta_{x}(\mathrm{~A}) & \text { otherwise } .\end{cases}
\end{aligned}
$$

The jump mechanism $\left(\lambda_{\mathrm{m}}, Q_{\mathrm{m}}\right)$ is minimal in the sense that if $\lambda_{\mathrm{m}}(t, x) \neq 0$, for $t \in$ $\mathbb{R}_{+}, x \in \mathrm{M}$, then $Q_{\mathrm{m}}(t, x,\{x\})=0$. As a consequence, if $\left(X_{t}\right)_{t \geqslant 0}$ is a PDMP with characteristics $\left(\varphi, \lambda_{\mathrm{m}}, Q_{\mathrm{m}}\right)$ and jump times $S_{k}, k \in \mathbb{N}$, then almost surely $X_{S_{k+1}} \neq$ $\varphi_{S_{k}, S_{k+1}}\left(X_{S_{k}}\right)$, and therefore $\left(X_{t}\right)_{t \geqslant 0}$ has no fantom jumps.

For all $(t, x, \mathrm{~A}) \in \mathbb{R}_{+} \times \mathrm{M} \times \mathcal{B}(\mathrm{M})$ and $i \in \llbracket 1, \ell \rrbracket$,

$$
Q_{i}(t, x, \mathrm{~A} \backslash\{x\})-\delta_{x}(\mathrm{~A}) Q_{i}(t, x, \mathrm{M} \backslash\{x\})=Q_{i}(t, x, \mathrm{~A})-\delta_{x}(\mathrm{~A}) .
$$

This means that, if $\left(\lambda_{i, 1}, Q_{i, 1}\right)_{i \in \llbracket 1, \ell_{1} \rrbracket}$ and $\left(\lambda_{i, 2}, Q_{i, 2}\right)_{i \in \llbracket 1, \ell_{2} \rrbracket}$ are two families of jump mechanisms satisfying (12), they have the same associated minimal jump mechanism. Therefore, the statement of Theorem 5 is equivalent to prove that, for all family $\left(\lambda_{i}, Q_{i}\right)_{i \in \llbracket 1, \ell \rrbracket}$, the associated minimal and total jump mechanisms are such that

$$
\operatorname{PDMP}\left(\varphi,\left(\lambda_{i}, Q_{i}\right)_{i \in \llbracket 1, \ell \rrbracket}, \mu_{0}\right)=\operatorname{PDMP}\left(\varphi, \lambda_{\mathrm{T}}, Q_{\mathrm{T}}, \mu_{0}\right)=\operatorname{PDMP}\left(\varphi, \lambda_{\mathrm{m}}, Q_{\mathrm{m}}, \mu_{0}\right) .
$$

We first show the first equality in the following lemma.

Lemma 6. Let $\left(\lambda_{\mathrm{T}}, Q_{\mathrm{T}}\right)$ be the total jump mechanism associated to $\left(\lambda_{i}, Q_{i}\right)_{i \in \llbracket 1, \ell \rrbracket}$. Then, for all flow $\varphi$ and $\mu_{0} \in \mathcal{P}(\mathrm{M}), \operatorname{PDMP}\left(\varphi,\left(\lambda_{i}, Q_{i}\right)_{i \in \llbracket 1, \ell \rrbracket}, \mu_{0}\right)=\operatorname{PDMP}\left(\varphi, \lambda_{\mathrm{T}}, Q_{\mathrm{T}}, \mu_{0}\right)$. 
Proof. Let $\left(X_{t}\right)_{t \geqslant 0}$ be a PDMP with characteristics $\left(\varphi,\left(\lambda_{i}, Q_{i}\right)_{i \in \llbracket 1, \ell \rrbracket}\right)$ and initial distribution $\mu_{0}$ defined by Construction 1 , and $\left(X_{k}^{\prime}, S_{k}\right)_{k \in \mathbb{N}}$ be its embedded chain. Since the process is completely determined by its embedded chain, and by the Markov property, it is sufficient to prove that the Markov kernel of $\left(X_{k}^{\prime}, S_{k}\right)_{k \in \mathbb{N}}$ is equal to the Markov kernel of the embedded chain associated to a PDMP with characteristics $\left(\varphi, \lambda_{\mathrm{T}}, Q_{\mathrm{T}}\right)$.

Summing out (3) over $j \in \llbracket 1, \ell \rrbracket$, we get for all $t \geqslant 0$ and $\mathrm{A} \in \mathcal{B}(\mathrm{M})$

$$
\begin{aligned}
& \mathbb{P}\left(X_{k+1}^{\prime} \in \mathrm{A}, S_{k+1} \leqslant t \mid \mathcal{F}_{k}\right) \\
& =\mathbb{1}_{\mathrm{M}}\left(X_{k}^{\prime}\right) \int_{S_{k}}^{t} \sum_{j=1}^{\ell} Q_{j}\left(s, \varphi_{S_{k}, s}\left(X_{k}^{\prime}\right), \mathrm{A}\right) \lambda_{j}\left(s, \varphi_{S_{k}, s}\left(X_{k}^{\prime}\right)\right) \exp \left[-\sum_{i=1}^{\ell} \int_{S_{k}}^{s} \lambda_{i}\left(u, \varphi_{S_{k}, u}\left(X_{k}^{\prime}\right)\right) \mathrm{d} u\right] \mathrm{d} s \\
& =\mathbb{1}_{\mathrm{M}}\left(X_{k}^{\prime}\right) \int_{S_{k}}^{t} Q_{\mathrm{T}}\left(s, \varphi_{S_{k}, s}\left(X_{k}^{\prime}\right), \mathrm{A}\right) \lambda_{\mathrm{T}}\left(s, \varphi_{S_{k}, s}\left(X_{k}^{\prime}\right)\right) \exp \left[-\int_{S_{k}}^{s} \lambda_{\mathrm{T}}\left(u, \varphi_{S_{k}, u}\left(X_{k}^{\prime}\right)\right) \mathrm{d} u\right] \mathrm{d} s,
\end{aligned}
$$

which concludes, since from (3) this is exactly the Markov kernel of the embedded chain associated to a PDMP with characteristics $\left(\varphi, \lambda_{\mathrm{T}}, Q_{\mathrm{T}}\right)$ defined by (13).

Before showing Theorem 5, we need the following technical lemma. In the sequel, Id denotes the identity Markov kernel, defined by $\operatorname{Id}(t, x, \mathrm{~A})=\delta_{x}(\mathrm{~A})$ for all $t \geqslant 0, x \in \mathrm{M}$, $\mathrm{A} \in \mathcal{B}(\mathrm{M})$. The following lemma gives a rigorous proof of the intuitive idea that adding fantom jumps does not change the distribution of the process.

Lemma 7. For any characteristics $(\varphi, \lambda, Q)$, jump rate $\lambda^{\prime}: \mathrm{M} \rightarrow \mathbb{R}_{+}$and $\mu_{0} \in \mathcal{P}(\mathrm{M})$,

$$
\operatorname{PDMP}\left(\varphi, \lambda, Q, \mu_{0}\right)=\operatorname{PDMP}\left(\varphi,\left\{(\lambda, Q),\left(\lambda^{\prime}, \mathrm{Id}\right)\right\}, \mu_{0}\right) .
$$

Proof. We consider $\left(Y_{t}\right)_{t \geqslant 0}$ a PDMP with characteristics $\left(\varphi,\left\{(\lambda, Q),\left(\lambda^{\prime}, \mathrm{Id}\right)\right\}\right)$ and initial distribution $\mu_{0}$ defined from random variables $W_{0}$ and $\left(E_{j, k}, U_{j, k}\right)_{j \in\{1,2\}, k \in \mathbb{N}}$ by Construction 2 , and its embedded chain $\left(Y_{k}^{\prime}, \tilde{S}_{k}, \tilde{I}_{k}\right)_{k \in \mathbb{N}}$. Let $R_{0}=0$ and, for $k \geqslant 1$, let $R_{k}=\inf \left\{\tilde{S}_{i}>R_{k-1}: i \in \mathbb{N}, \tilde{I}_{i}=1\right\}$ be the $k^{\text {th }}$ jump of type 1 (i.e. associated with the jump mechanism $(\lambda, Q))$. For $i \geqslant 1$ such that $\tilde{I}_{i}=2$, the $i^{\text {th }}$ jump is a fantom one, i.e. $Y_{\tilde{S}_{i}}=\varphi_{\tilde{S}_{i-1}, \tilde{S}_{i}}\left(Y_{\tilde{S}_{i-1}}\right)$. By the flow property $\varphi_{s, u} \circ \varphi_{t, s}=\varphi_{t, u}$, this implies that $Y_{t}=\varphi_{R_{k}, t}\left(Y_{R_{k}}\right)$ for all $k \in \mathbb{N}$ and $t \in\left[R_{k}, R_{k+1} \wedge \tau_{\infty}(Y)\right)$.

If $k \in \mathbb{N}$ is such that $R_{k}<\infty$, then $\left\{\varphi_{R_{k}, t}\left(Y_{R_{k}}\right): t \in\left[R_{k},\left(a+R_{k}\right) \wedge R_{k+1}\right]\right\}$ is a compact set of $\mathrm{M}$, on which $\lambda^{\prime}$ is bounded (as a locally bounded function), for any $a>0$. Hence, there cannot be an infinite number of jump of second type between times $R_{k}$ and $\left(a+R_{k}\right) \wedge R_{k+1}$, for any $a>0$. In particular, necessarily, $\sup \left\{R_{k}: k \in \mathbb{N}\right\}=\sup \left\{\tilde{S}_{k}:\right.$ $k \in \mathbb{N}\}=\tau_{\infty}(Y)$. Indeed, for any $k \in \mathbb{N}, \tilde{S}_{k} \leqslant R_{k}$ by definition and if $R_{k}<+\infty$, for any $a>0$, almost surely, there exists $n_{k} \in \mathbb{N}$ such that $\tilde{S}_{n_{k}} \geqslant\left(R_{k}+a\right) \wedge R_{k+1}$. As a consequence,

$$
Y_{t}=\varphi_{R_{k}, t}\left(Y_{R_{k}}\right) \text { holds for all } k \in \mathbb{N} \text { and } t \in\left[R_{k}, R_{k+1}\right) \text { on }\left\{R_{k}<+\infty\right\} .
$$

Define for all $k \in \mathbb{N}^{*}, N_{k}=\inf \left\{i>N_{k-1}: \tilde{I}_{i}=1\right\}$, setting $N_{0}=0$. Construction 2 implies by a straightforward induction that for any $k \in \mathbb{N}$, on $\left\{R_{k}<+\infty\right\} \cap\left\{N_{k+1}<\right.$ $+\infty\}$

$$
R_{k+1}=\inf \left\{t \geqslant R_{k}: E_{1, k+1}<\int_{R_{k}}^{t} \lambda\left(s, \varphi_{R_{k}, s}\left(Y_{R_{k}}\right)\right) \mathrm{d} s\right\}
$$


In addition, for all $k \in \mathbb{N}$,

$$
\begin{aligned}
& \left\{R_{k}<+\infty\right\} \cap\left\{N_{k+1}=+\infty\right\} \\
& =\left\{R_{k}<+\infty\right\} \cap\left\{N_{k+1}=+\infty\right\} \cap\left\{\bigcap_{i \geqslant N_{k}}\left\{\tilde{H}_{1, i+1}>\int_{S_{i}}^{t} \lambda\left(s, \varphi_{S_{i}, s}\left(Y_{S_{i}}\right)\right) \mathrm{d} s\right\}\right\} \\
& =\left\{R_{k}<+\infty\right\} \cap\left\{N_{k+1}=+\infty\right\} \cap\left\{E_{1, k+1} \geqslant \int_{R_{k}}^{+\infty} \lambda\left(s, \varphi_{R_{k}, s}\left(Y_{R_{k}}\right)\right) \mathrm{d} s\right\} .
\end{aligned}
$$

Therefore, on $\left\{R_{k}<+\infty\right\} \cap\left\{N_{k+1}=+\infty\right\}$,

$$
R_{k+1}=+\infty=\inf \left\{t \geqslant R_{k}: E_{1, k+1}<\int_{R_{k}}^{t} \lambda\left(s, \varphi_{R_{k}, s}\left(Y_{R_{k}}\right)\right) \mathrm{d} s\right\},
$$

and, if $R_{k+1}<\infty, Y_{R_{k+1}}=\mathbf{G}_{1}\left(R_{k+1}, \varphi_{R_{k}, R_{k+1}}\left(Y_{R_{k}}\right), U_{1, k}\right)$.

Therefore, denoting $Z_{k}^{\prime}=Y_{R_{k}}$ for all $k \in \mathbb{N}$, then $\left(Z_{k}^{\prime}, R_{k}\right)$ is the embedded chain associated to a PDMP $\left(Z_{t}\right)_{t \geqslant 0}$ with characteristics $(\varphi, \lambda, Q)$ and constructed with the random variables $W_{0}$ and $\left(E_{1, k}, U_{1, k}\right)_{k \geqslant 0}$ (through either Construction 1 or 2 , since there is only one jump mechanism so that both coincides). Finally, for all $k \in \mathbb{N}$ and $t \in\left[R_{k}, R_{k+1}\right), Z_{t}=\varphi_{R_{k}, t}\left(Z_{k}^{\prime}\right)=Y_{t}$ by (14), which concludes the proof.

Proof of Theorem 5. As previously mentioned, to show Theorem 5, it is sufficient to prove that, for all differential flow $\varphi$, initial distribution $\mu_{0}$ and family of jump mechanisms $\left(\lambda_{i}, Q_{i}\right)_{i \in \llbracket 1, \ell \rrbracket}$,

$$
\operatorname{PDMP}\left(\varphi,\left(\lambda_{i}, Q_{i}\right)_{i \in \llbracket 1, \ell \rrbracket}, \mu_{0}\right)=\operatorname{PDMP}\left(\varphi, \lambda_{\mathrm{T}}, Q_{\mathrm{T}}, \mu_{0}\right)=\operatorname{PDMP}\left(\varphi, \lambda_{\mathrm{m}}, Q_{\mathrm{m}}, \mu_{0}\right)
$$

holds, where $\left(\lambda_{\mathrm{T}}, Q_{\mathrm{T}}\right)$ and $\left(\lambda_{\mathrm{m}}, Q_{\mathrm{m}}\right)$ are the associated total and minimal jump mechanisms defined by (13). The first identity is given by Lemma 6 , therefore it remains to show the second one.

By Lemma 7 , since $\lambda_{\mathrm{T}}-\lambda_{\mathrm{m}}$ is by definition a jump rate (i.e. a positive and locally bounded measurable function), we get for all differential flow $\varphi$ and initial distribution $\mu_{0}$ that

$$
\operatorname{PDMP}\left(\varphi, \lambda_{\mathrm{m}}, Q_{\mathrm{m}}, \mu_{0}\right)=\operatorname{PDMP}\left(\varphi,\left\{\left(\lambda_{\mathrm{m}}, Q_{\mathrm{m}}\right),\left(\lambda_{\mathrm{T}}-\lambda_{\mathrm{m}}, \mathrm{Id}\right)\right\}, \mu_{0}\right) .
$$

The proof is concluded upon noting that the total jump mechanism associated with $\left\{\left(\lambda_{\mathrm{m}}, Q_{\mathrm{m}}\right),\left(\lambda_{\mathrm{T}}-\lambda_{\mathrm{m}}, \mathrm{Id}\right)\right\}$ is equal to $\left(\lambda_{\mathrm{T}}, Q_{\mathrm{T}}\right)$ and using Lemma 6 again.

Example - Bouncy Particle Sampler. By Theorem 5, the BPS process defined in Example 1 is a PDMP with characteristics $(\varphi, \lambda, Q)$, where for all $t \geqslant 0,(x, y) \in \mathbb{R}^{d} \times \mathrm{Y}$, and $\mathrm{A} \in \mathcal{B}\left(\mathbb{R}^{d} \times \mathrm{Y}\right), \varphi_{t}(x, y)=(x+t y, y)$,

$$
\lambda(x, y)=\langle\nabla U(x), y\rangle_{+}+\lambda_{\mathrm{c}}
$$

and

$$
Q((x, y), \mathrm{A})=\lambda^{-1}(x, y)\left\{\langle\nabla U(x), y\rangle_{+} \delta_{(x, \mathrm{R}(x, y))}(\mathrm{A})+\lambda_{\mathrm{c}}\left(\boldsymbol{\delta}_{x} \otimes \mu_{\mathrm{v}}\right)(\mathrm{A})\right\},
$$

where $\mathrm{R}$ is defined in (5) with $g=\nabla U$. 


\section{NON-EXPLOSION}

It is generally easier to prove that a given particular PDMP is non-explosive than to provide good general conditions that ensure non-explosion for PDMPs. Nevertheless, we give here two results on that topic that will prove useful in the rest of this work, and may be of interest in other situations.

Proposition 8. Let $\left(X_{t}\right)_{t \geqslant 0}$ be a PDMP with characteristics $\left(\varphi,\left(\lambda_{i}, Q_{i}\right)_{i \in \llbracket 1, \ell \rrbracket}\right)$ and initial distribution $\mu_{0}$ given by Construction 1 for some random variables $W_{0}$ and $\left(\left(E_{j, k}\right)_{j \in \llbracket 1, \ell \rrbracket}, U_{k}\right)_{k \in \mathbb{N}}$. For all $M>0$, let $\left(X_{t}^{M}\right)_{t \geqslant 0}$ be a PDMP with characteristics $\left(\varphi,\left(M \wedge \lambda_{i}, Q_{i}\right)_{i \in \llbracket 1, \ell \rrbracket}\right)$ and initial distribution $\mu_{0}$ be given by Construction 1 for the same random variables $W_{0}$ and $\left(\left(E_{j, k}\right)_{j \in \llbracket 1, \ell \rrbracket}, U_{k}\right)_{k \in \mathbb{N}}$ as $\left(X_{t}\right)_{t \geqslant 0}$. The process $\left(X_{t}\right)_{t \geqslant 0}$ is non-explosive if and only if, for all $t \geqslant 0$,

$$
\lim _{M \rightarrow+\infty} \mathbb{P}\left(X_{s}=X_{s}^{M}, \text { for all } s \in[0, t]\right)=1 .
$$

Proof. Suppose that $\left(X_{t}\right)_{t \geqslant 0}$ is non-explosive. Then for almost all $\omega \in \Omega$, the process only jumps a finite number of time between times 0 and $t$, so that $\left\{X_{s}: s \in\right.$ $[0, t]\}$ is a compact set of $M$. Since the rate jumps are locally bounded, $\lambda_{\star}(\omega)=$ $\sup \left\{\sum_{j=1}^{\ell} \lambda_{j}\left(s, X_{s}\right): s \in[0, t]\right\}$ is finite for almost all $\omega \in \Omega$. We get for all $\omega \in \Omega$ and $M>\lambda_{\star}(\omega)$, by definition that $\sup _{s \in[0, t]}\left|X_{s}-X_{s}^{M}\right|=0$ and therefore, for almost all $\omega \in \Omega, \lim _{M \rightarrow+\infty} \mathbb{1}_{\{0\}}\left(\sup _{s \in[0, t]}\left|X_{s}-X_{s}^{M}\right|\right)=1$. Thus, since $\left\{X_{s}=X_{s}^{M}\right.$, for all $s \in$ $[0, t]\}=\left\{\sup _{s \in[0, t]}\left|X_{s}-X_{s}^{M}\right|=0\right\}$, the proof is concluded using the Lebesgue dominated convergence theorem.

Now, to prove the converse, remark that $\left(X_{t}^{M}\right)_{t \geqslant 0}$ is non-explosive for all $M>0$, since its jump rates are bounded. In particular, $X_{t}^{M} \in \mathrm{M}$ for all $t \geqslant 0$, so that

$$
\mathbb{P}\left(\tau_{\infty}(X)>t\right)=\mathbb{P}\left(X_{t} \in \mathrm{M}\right) \geqslant \mathbb{P}\left(X_{t}=X_{t}^{M}\right)
$$

for all $M>0$. The conclusion then follows taking $M \rightarrow+\infty$.

In particular, Proposition 8 implies that, if $\left(\varphi,\left(\lambda_{i}, Q_{i}\right)_{i \in \llbracket 1, \ell \rrbracket}\right)$ is non explosive, denoting by $\left(P_{s, t}\right)_{t \geqslant s \geqslant 0}$ and $\left(P_{s, t}^{M}\right)_{t \geqslant s \geqslant 0}$ the Markov semi-group associated to characteristics $\left(\varphi,\left(\lambda_{i}, Q_{i}\right)_{i \in \llbracket 1, \ell \rrbracket}\right)$ and $\left(\varphi,\left(M \wedge \lambda_{i}, Q_{i}\right)_{i \in \llbracket 1, \ell \rrbracket}\right), M>0$ then, since for all $\mu_{0} \in \mathcal{P}(\mathrm{M})$ and all $t \geqslant 0$,

$$
\left\|\mu_{0} P_{0, t}-\mu_{0} P_{0, t}^{M}\right\|_{\mathrm{TV}}=\sup _{\mathrm{A} \in \mathcal{B}(\mathrm{M})}\left|\mathbb{P}\left(X_{t} \in \mathrm{A}\right)-\mathbb{P}\left(X_{t}^{M} \in \mathrm{A}\right)\right| \leqslant 2 \mathbb{P}\left(X_{t} \neq X_{t}^{M}\right),
$$

we get $\lim _{M \rightarrow+\infty}\left\|\mu_{0} P_{0, t}-\mu_{0} P_{0, t}^{M}\right\|_{\mathrm{TV}}=0$.

The second result concerning non-explosion of PDMPs is the following:

Proposition 9. Let $\left(\varphi,\left(\lambda_{i}, Q_{i}\right)_{i \in \llbracket 1, \ell \rrbracket}\right)$ be homogeneous characteristics with $\ell>1$. Assume that the characteristics $\left(\varphi,\left(\lambda_{i}, Q_{i}\right)_{i \in \llbracket 1, \ell-1 \rrbracket}\right)$ are non-explosive and $\left\|\lambda_{\ell}\right\|_{\infty}<+\infty$. Then $\left(\varphi,\left(\lambda_{i}, Q_{i}\right)_{i \in \llbracket 1, \ell \rrbracket}\right)$ are non-explosive as well.

Proof. Let $\mu_{0} \in \mathcal{P}(\mathrm{M})$ and $\left(Y_{t}\right)_{t \geqslant 0}$ be a PDMP with characteristics $\left(\varphi,\left(\lambda_{i}, Q_{i}\right)_{i \in \llbracket 1, \ell \rrbracket}\right)$ and initial distribution $\mu_{0}$ given by Construction 2 based on random variables $W_{0}$ and $\left(E_{j, k}, U_{j, k}\right)_{j \in \llbracket 1, \ell \rrbracket, k \in \mathbb{N}}$. Let $\left(Y_{k}^{\prime}, \tilde{S}_{k}, \tilde{I}_{k}\right)_{k \in \mathbb{N}}$ be the corresponding embedded chain. First, 
consider the decomposition

(16)

$\mathbb{P}\left(\sup _{n \in \mathbb{N}} \tilde{S}_{n}<+\infty\right)=\mathbb{P}\left(\sup _{n \in \mathbb{N}} \tilde{S}_{n}<+\infty, \sup _{n \in \mathbb{N}^{*}} \tilde{N}_{\ell, n}=+\infty\right)+\mathbb{P}\left(\sup _{n \in \mathbb{N}} \tilde{S}_{n}<+\infty, \sup _{n \in \mathbb{N}^{*}} \tilde{N}_{\ell, n}<+\infty\right)$.

Let us show that both terms of the right-hand-side are equal to 0 .

Define recursively $\left(N_{n}^{(\ell)}\right)_{n \in \mathbb{N}}$ by $N_{0}^{(\ell)}=0$ and for all $n \in \mathbb{N}, N_{n+1}^{(\ell)}=\inf \left\{k>N_{n}^{(\ell)}\right.$ : $\left.\tilde{I}_{k}=\ell\right\}$ Note that for any $n, k \in \mathbb{N}$ and

$$
N_{n}^{(\ell)}=k \text { if and only if } \tilde{N}_{\ell, k}=n, \quad \tilde{S}_{N_{n}^{(\ell)}}=\tilde{S}_{\ell, N_{n}^{(\ell)}} .
$$

Then, on $\left\{\sup _{n \in \mathbb{N}^{*}} \tilde{N}_{\ell, n}=+\infty\right\}$, for all $n \in \mathbb{N}, N_{n}^{(\ell)}<+\infty$ almost surely. Hence, on $\left\{\sup _{n \in \mathbb{N}^{*}} \tilde{N}_{n, \ell}=+\infty\right\}$, for all $n \in \mathbb{N}^{*}$, by definition we have almost surely

$$
\sup _{k \in \mathbb{N}^{*}} \tilde{S}_{k} \geqslant \sum_{k=1}^{n}\left\{\tilde{S}_{N_{k}^{(\ell)}}-\tilde{S}_{N_{k-1}^{(\ell)}}\right\} \geqslant \sum_{i=1}^{n}\left(E_{\ell, i} /\left\|\lambda_{\ell}\right\|_{\infty}\right),
$$

where the last inequality follows from the bound on $\left\{N_{k}^{(\ell)}<+\infty\right\}$,

$$
\begin{aligned}
\tilde{S}_{N_{k}^{(\ell)}}=\tilde{S}_{\ell, N_{k}^{(\ell)}} & =\inf \left\{t>\tilde{S}_{N_{k}^{(\ell)}-1}: H_{\ell, N_{k}^{(\ell)}}<\int_{\tilde{S}_{N_{k}^{(\ell)}-1}}^{t} \lambda_{\ell}\left(X_{s}\right) \mathrm{d} s\right\} \\
& =\inf \left\{t>\tilde{S}_{N_{k-1}^{(\ell)}}: E_{\ell, k}<\int_{\tilde{S}_{N_{k-1}^{(\ell)}}}^{t} \lambda_{\ell}\left(X_{s}\right) \mathrm{d} s\right\} \geqslant \tilde{S}_{N_{k-1}^{(\ell)}}+E_{\ell, k} /\left\|\lambda_{\ell}\right\|_{\infty} .
\end{aligned}
$$

Therefore by the law of large number,

$$
\mathbb{P}\left(\sup _{n \in \mathbb{N}} \tilde{S}_{n}<+\infty, \sup _{n \in \mathbb{N}^{*}} \tilde{N}_{\ell, n}=+\infty\right) \leqslant \inf _{n \in \mathbb{N}^{*}} \mathbb{P}\left(\sum_{i=1}^{n}\left(E_{\ell, i} /\left\|\lambda_{\ell}\right\|_{\infty}\right)<+\infty\right)=0 .
$$

We bound now the second term in (16). Let $k \in \mathbb{N}^{*}$. Note that by Construction 2, on $\left\{\sup _{n \in \mathbb{N}} \tilde{N}_{\ell, n}=k\right\}$, for all $i \in \llbracket 1, k-1 \rrbracket,\left\{X_{t}: t \in\left[\tilde{S}_{N_{i}^{(\ell)}}, \tilde{S}_{N_{i+1}^{(\ell)}}\right)\right\}$ is a PDMP with characteristics $\left(\varphi,\left(\lambda_{i}, Q_{i}\right)_{i \in \llbracket 1, \ell-1 \rrbracket}\right)$ with initial data $X_{\tilde{S}_{N_{i}^{(\ell)}}}$. Then, by (17), the Markov property and an immediate induction using that $\left(\varphi,\left(\lambda_{i}^{i}, Q_{i}\right)_{i \in \llbracket 1, \ell-1 \rrbracket}\right)$ is non explosive, we have that on $\left\{\sup _{n \in \mathbb{N}^{*}} \tilde{N}_{\ell, n}=k\right\}$, almost surely $X_{\tilde{S}_{N_{i}(\ell)}} \in \mathrm{M}$, for $i \in \llbracket 1, k \rrbracket$. The proof is then concluded since, using that $\left(\varphi,\left(\lambda_{i}, Q_{i}\right)_{i \in \llbracket 1, \ell-1 \rrbracket}\right)$ is non explosive, Construction 2 and the Markov property again on $\left\{\sup _{n \in \mathbb{N}} \tilde{N}_{\ell, n}=k\right\},\left\{X_{t}: t \in\left[\tilde{S}_{N_{k}^{(\ell)}},+\infty\right)\right\}$ is a PDMP with characteristics $\left(\varphi,\left(\lambda_{i}, Q_{i}\right)_{i \in \llbracket 1, \ell-1 \rrbracket}\right)$ started at $X_{\tilde{S}_{N_{k}^{(\ell)}}}$.

Let us come back to our main example.

\section{Example - Bouncy Particle Sampler.}

Proposition 10. The BPS process defined in Example 1 is non-explosive for any initial distribution. 
Proof. Using the notation of Example 1, consider $\left(\bar{X}_{t}, \bar{Y}_{t}\right)_{t \geqslant 0}$ the PDMP on $\mathbb{R}^{d} \times \mathrm{Y}$ with characteristics $\left(\varphi, \lambda_{1}, Q_{1}\right)$ and initial condition $\left(\bar{X}_{0}, \bar{Y}_{0}\right)=(x, y) \in \mathrm{M}$ defined by Construction 1 from an i.i.d. sequence of random variables $\left(E_{k}, U_{k}\right)_{k \in \mathbb{N}}$, associated with the sequence of jump times $\left(S_{k}\right)_{k \in \mathbb{N}}$. Note that almost surely $\left\|\bar{Y}_{t}\right\|=\|y\|$ for all $t \in\left[0, \sup _{n \in \mathbb{N}} S_{n}\right)$, so that $\left\|\bar{X}_{t}-x\right\| \leqslant t\|y\|$ and

$$
\lambda_{1}\left(\bar{X}_{t}, \bar{Y}_{t}\right) \leqslant C(t)=\|y\| \sup \left\{\left\|\nabla U\left(x^{\prime}\right)\right\|: x^{\prime} \in \mathbb{R}^{d},\left\|x^{\prime}-x\right\| \leqslant t\|y\|\right\} .
$$

Therefore on $\left\{\sup _{n \in \mathbb{N}} S_{n}<+\infty\right\}$, by Construction 1 almost surely there exists $C \geqslant 0$ $(C=C(a)$ for some $a>0)$ such that

$$
S_{n+1}-S_{n} \geqslant E_{n} /(C+1) \text { for all } n \in \mathbb{N}^{*} \text {. }
$$

Then, we have on $\left\{\sup _{n \in \mathbb{N}} S_{n}<+\infty\right\}$, that almost surely there exists $C \geqslant 0$ such that

$$
+\infty>\sup _{n \in \mathbb{N}} S_{n}=\sum_{n \in \mathbb{N}}\left\{S_{n+1}-S_{n}\right\} \geqslant \sum_{n \in \mathbb{N}^{*}}\left\{E_{n} /(C+1)\right\} .
$$

As a result,

$$
\mathbb{P}\left(\sup _{n \in \mathbb{N}} S_{n}<+\infty\right) \leqslant \mathbb{P}\left(\bigcup_{\ell \in \mathbb{N}^{*}}\left\{\sum_{n \in \mathbb{N}^{*}} E_{n} /(\ell+1)<+\infty\right\}\right) \leqslant 0 .
$$

It follows that the PDMP with characteristics $\left(\varphi, \lambda_{1}, Q_{1}\right)$ is non-explosive. By Proposition 9, the BPS is non-explosive.

\section{Comparison of PDMP via Synchronous coupling}

A synchronous coupling is a coupling between two PDMPs that ensures that, as long as possible, the two corresponding processes jump at the same times and to the same location. This construction yields total variation estimates between the marginal distributions of different PDMPs sharing the same differential flow.

The result of this section is crucial in many aspects: first, it gives stability estimates with respect to the jump rates and the underlying Markov kernel for a modification of a PDMP for example for an approximate thinning procedure. Second, it gives a way to design smooth (in some sense) approximations of a given (non-smooth) PDMP, which will be an essential tools to verify assumptions for the BPS in the following sections. The main goal of this section is to prove the following:

Theorem 11. Let $\left(P_{s, t}^{(1)}\right)_{t \geqslant s \geqslant 0}$ and $\left(P_{s, t}^{(2)}\right)_{t \geqslant s \geqslant 0}$ be two non-explosive PDMP semigroups with characteristics $\left(\varphi, \lambda^{(1)}, Q^{(1)}\right)$ and $\left(\varphi, \lambda^{(2)}, Q^{(2)}\right)$ respectively. Suppose that there exists a measurable $g: \mathbb{R}_{+} \rightarrow \mathbb{R}_{+}$satisfying for all $t \geqslant 0$,

$$
g(t) \geqslant \sup _{\substack{x \in \mathrm{M}, \mathrm{A} \in \mathcal{B}(\mathrm{M})}}\left\{\lambda^{(1)}(t, x) \wedge \lambda^{(2)}(t, x)\left|Q^{(1)}(t, x, \mathrm{~A})-Q^{(2)}(t, x, \mathrm{~A})\right|\right\}+\sup _{x \in \mathrm{M}}\left|\lambda^{(1)}(t, x)-\lambda^{(2)}(t, x)\right|
$$

or alternatively

$$
g(t) \geqslant \sup _{\substack{x \in \mathbb{M}, \mathrm{A} \in \mathcal{B}(\mathrm{M})}}\left|\lambda^{(1)}(t, x)\left(Q^{(1)}(t, x, \mathrm{~A})-\delta_{x}(\mathrm{~A})\right)-\lambda^{(2)}(t, x)\left(Q^{(2)}(t, x, \mathrm{~A})-\delta_{x}(\mathrm{~A})\right)\right| .
$$


Then for all $t \geqslant 0$ and $x \in \mathrm{M},\left\|\delta_{x} P_{0, t}^{(1)}-\delta_{x} P_{0, t}^{(2)}\right\|_{\mathrm{TV}} \leqslant 2\left\{1-\exp \left(-\int_{0}^{t} g(s) \mathrm{d} s\right)\right\}$.

Remark 12. Note that if $\left(\lambda^{(i)}\right)_{i=1,2}$ and $\left(Q^{i}\right)_{i=1,2}$ are two locally bounded jump rates and Markov kernels respectively, then

$$
\begin{aligned}
& \sup _{\substack{x \in \mathbb{M}, \mathrm{A} \in \mathcal{B}(\mathrm{M})}}\left\{\lambda^{(1)}(t, x) \wedge \lambda^{(2)}(t, x)\left|Q^{(1)}(t, x, \mathrm{~A})-Q^{(2)}(t, x, \mathrm{~A})\right|\right\} \\
& \quad \leqslant \sup _{\substack{x \in \mathbb{M}, \mathbf{A} \in \mathcal{B}(\mathrm{M})}}\left\{\left|\lambda^{(1)}(t, x) Q^{(1)}(t, x, \mathrm{~A})-\lambda^{(2)}(t, x) Q^{(2)}(t, x, \mathrm{~A})\right|\right\} .
\end{aligned}
$$

Indeed, let $x \in \mathrm{M}$ and $\mathrm{A} \in \mathcal{B}(\mathrm{M})$. Without loss of generality, we can assume that $\lambda^{(1)}(t, x) \geqslant \lambda^{(2)}(t, x)$. If $Q^{(1)}(t, x, \mathrm{~A}) \geqslant Q^{(2)}(t, x, \mathrm{~A})$, then we have

$$
\begin{aligned}
& \lambda^{(1)}(t, x) \wedge \lambda^{(2)}(t, x)\left|Q^{(1)}(t, x, \mathrm{~A})-Q^{(2)}(t, x, \mathrm{~A})\right| \\
\leqslant & \lambda^{(1)}(t, x) Q^{(1)}(t, x, \mathrm{~A})-\lambda^{(2)}(t, x) Q^{(2)}(t, x, \mathrm{~A})=\left|\lambda^{(1)}(t, x) Q^{(1)}(t, x, \mathrm{~A})-\lambda^{(2)}(t, x) Q^{(2)}(t, x, \mathrm{~A})\right| .
\end{aligned}
$$

Otherwise $Q^{(1)}\left(t, x, \mathrm{~A}^{\mathrm{c}}\right) \geqslant Q^{(2)}\left(t, x, \mathrm{~A}^{\mathrm{c}}\right)$ and we get

$\lambda^{(1)}(t, x) \wedge \lambda^{(2)}(t, x)\left|Q^{(1)}(t, x, \mathrm{~A})-Q^{(2)}(t, x, \mathrm{~A})\right| \leqslant\left|\lambda^{(1)}(t, x) Q^{(1)}\left(t, x, \mathrm{~A}^{\mathrm{c}}\right)-\lambda^{(2)}(t, x) Q^{(2)}\left(t, x, \mathrm{~A}^{\mathrm{c}}\right)\right|$.

Therefore, for all $x \in \mathrm{M}$ and $\mathrm{A} \in \mathcal{B}(\mathrm{M})$,

$\lambda^{(1)}(t, x) \wedge \lambda^{(2)}(t, x)\left|Q^{(1)}(t, x, \mathrm{~A})-Q^{(2)}(t, x, \mathrm{~A})\right| \leqslant \sup _{\tilde{\tilde{A}} \in \mathcal{B}(\mathrm{M})}\left|\lambda^{(1)}(t, x) Q^{(1)}(t, x, \tilde{\mathrm{A}})-\lambda^{(2)}(t, x) Q^{(2)}(t, x, \tilde{\mathrm{A}})\right|$,

which implies (20). Therefore, to establish that (18), it is sufficient to show that there exists a measurable function $g: \mathbb{R}_{+} \rightarrow \mathbb{R}_{+}$such that for all $t \in \mathbb{R}_{+}$,

$$
g(t) \geqslant 2 \sup _{\substack{x \in \mathbb{M}, \mathrm{A} \in \mathcal{B}(\mathrm{M})}}\left\{\left|\lambda^{(1)}(t, x) Q^{(1)}(t, x, \mathrm{~A})-\lambda^{(2)}(t, x) Q^{(2)}(t, x, \mathrm{~A})\right|\right\},
$$

Conversely, we easily get for all $t \in \mathbb{R}_{+}$,

$$
\begin{aligned}
& (22) \sup _{\substack{x \in \mathbb{M}, \mathrm{A} \in \mathcal{B}(\mathrm{M})}}\left\{\left|\lambda^{(1)}(t, x) Q^{(1)}(t, x, \mathrm{~A})-\lambda^{(2)}(t, x) Q^{(2)}(t, x, \mathrm{~A})\right|\right\} \\
& \leqslant \sup _{\substack{x \in \mathbb{M}, \mathbf{A} \in \mathcal{B}(\mathrm{M})}}\left\{\lambda^{(1)}(t, x) \wedge \lambda^{(2)}(t, x)\left|Q^{(1)}(t, x, \mathrm{~A})-Q^{(2)}(t, x, \mathrm{~A})\right|\right\}+\sup _{x \in \mathbb{M}}\left|\lambda^{(1)}(t, x)-\lambda^{(2)}(t, x)\right| .
\end{aligned}
$$

Therefore (18) and (21) are essentially equivalent up to a factor 2.

The proof of Theorem 11 relies on the construction of the Markovian synchronous coupling between $\left(P_{s, t}^{(1)}\right)_{t \geqslant s \geqslant 0}$ and $\left(P_{s, t}^{(2)}\right)_{t \geqslant s \geqslant 0}$. More precisely, we want to construct a $\operatorname{PDMP}\left(X_{t}, Y_{t}\right)_{t \geqslant 0}$ on $\mathrm{M}^{2}$ starting from $(x, y) \in \mathrm{M}^{2}$ such that the distributions of $\left(X_{t}\right)_{t \geqslant 0}$ and $\left(Y_{t}\right)_{t \geqslant 0}$ are $\operatorname{PDMP}\left(\varphi, \lambda^{(1)}, Q^{(1)}, \delta_{x}\right)$ and $\operatorname{PDMP}\left(\varphi, \lambda^{(2)}, Q^{(2)}, \delta_{y}\right)$ respectively. In the case where $x=y$, the synchronous coupling attempts to keep $X_{s}=Y_{s}$ for all $s \in[0, t]$ by ensuring that, as much as possible, both processes jump at the same time and, when they do, jump as much as possible to the same point. Let us give the formal definition of $\left(X_{t}, Y_{t}\right)_{t \geqslant 0}$. 
First, by [24, Corollary 5.22] or [11, Theorem 19.1.12], there exists a jump kernel $K_{0}$ on $\mathrm{M}^{2}$, such that for all $t \in \mathbb{R}_{+}$and $(x, y) \in \mathrm{M}^{2}, K_{0}(t,(x, y), \cdot)$ is an optimal transference plane of $Q^{(1)}(t, x, \cdot)$ and $Q^{(2)}(t, y, \cdot)$ for the total variation, i.e. for any $\mathrm{A} \in \mathcal{B}(\mathrm{M})$, $K_{0}(t,(x, y), \mathrm{A} \times \mathrm{M})=Q^{(1)}(x, \mathrm{~A}), K_{0}(t,(x, y), \mathrm{M} \times \mathrm{A})=Q^{(2)}(y, \mathrm{~A})$ and

$$
2 K_{0}\left(t,(x, y), \Delta_{\mathrm{M}}^{c}\right)=\left\|Q^{(1)}(t, x, \cdot)-Q^{(2)}(t, y, \cdot)\right\|_{\mathrm{TV}} .
$$

Define, for $i=0,1,2$ and $j=1,2$ the jump rate $r_{i}$ and the jump kernel $K_{j}$ on $\mathrm{M}^{2}$ as follows: for $t \in \mathbb{R}_{+},(x, y) \in \mathrm{M}^{2}$ and $\mathrm{A}, \mathrm{B} \in \mathcal{B}(\mathrm{M})$,

$$
\begin{aligned}
& r_{0}(t,(x, y))=\lambda^{(1)}(t, x) \wedge \lambda^{(2)}(t, y), \\
& r_{1}(t,(x, y))=\left(\lambda^{(1)}(t, x)-\lambda^{(2)}(t, y)\right)_{+}, \quad K_{1}(t,(x, y), \mathrm{A} \times \mathrm{B})=Q^{(1)}(t, x, \mathrm{~A}) \boldsymbol{\delta}_{y}(\mathrm{~B}), \\
& r_{2}(t,(x, y))=\left(\lambda^{(2)}(t, x)-\lambda^{(1)}(t, y)\right)_{+}, \quad K_{2}(t,(x, y), \mathrm{A} \times \mathrm{B})=\delta_{x}(\mathrm{~A}) Q^{(2)}(t, y, \mathrm{~B}) .
\end{aligned}
$$

Let $\varphi^{\otimes}$ be the flow on $\mathrm{M}^{2}$ defined for all $t \in \mathbb{R}_{+}$and $(x, y) \in \mathrm{M}^{2}$ by

$$
\varphi^{\otimes}(t,(x, y))=(\varphi(t, x), \varphi(t, y)) .
$$

Lemma 13. Let $(x, y) \in \mathrm{M}^{2}$ and $\left(X_{t}, Y_{t}\right)_{t \geqslant 0}$ be a PDMP on $\mathrm{M}^{2}$ with initial distribution $\boldsymbol{\delta}_{(x, y)}$ and characteristics $\left(\varphi^{\otimes},\left(r_{i}, K_{i}\right)_{i \in \llbracket 0,2 \rrbracket}\right)$. Suppose that it is non explosive. Then $\left(X_{t}\right)_{t \geqslant 0}$ and $\left(Y_{t}\right)_{t \geqslant 0}$ have distributions $\operatorname{PDMP}\left(\varphi, \lambda^{(1)}, Q^{(1)}, \delta_{x}\right)$ and $\operatorname{PDMP}\left(\varphi, \lambda^{(2)}, Q^{(2)}, \delta_{y}\right)$ respectively.

As a consequence, $\left(X_{t}, Y_{t}\right)_{t \geqslant 0}$ is referred to as a synchronous coupling of $\left(\delta_{x} P_{0, t}^{(1)}\right)_{t \geqslant 0}$ and $\left(\delta_{x} P_{0, t}^{(2)}\right)_{t \geqslant 0}$.

Proof. We only show the result for $\left(X_{t}\right)_{t \geqslant 0}$, the case for $\left(Y_{t}\right)_{t \geqslant 0}$ being similar. Consider the Markov kernel on $\mathrm{M}^{2} \times \mathcal{B}\left(\mathrm{M}^{2}\right)$ defined for all $(x, y) \in \mathrm{M}^{2}$ and $\mathrm{A} \in \mathcal{B}\left(\mathrm{M}^{2}\right)$ by

$\tilde{K}(t,(x, y), \mathrm{A})= \begin{cases}\delta_{(x, y)}(\mathrm{A}) & \text { if } r_{0}(x, y)+r_{1}(x, y)=0 \\ {\left[r_{0}(x, y) K_{0}(t,(x, y), \mathrm{A})+r_{1}(x, y) K_{1}(t,(x, y), \mathrm{A})\right] /\left[r_{0}(x, y)+r_{1}(x, y)\right]}\end{cases}$

otherwise .

By Theorem 5 and since $\lambda^{(1)}=r_{0}+r_{1}$, we have that $\operatorname{PDMP}\left(\varphi^{\otimes}, \lambda^{(1)}, \tilde{K}, r_{2}, K_{2}, \boldsymbol{\delta}_{x} \otimes \boldsymbol{\delta}_{y}\right)=$ $\operatorname{PDMP}\left(\varphi^{\otimes},\left(r_{i}, K_{i}\right)_{i \in \llbracket 0,2 \rrbracket}, \delta_{x} \otimes \delta_{y}\right)$. As a consequence, we can assume that $\left(X_{t}, Y_{t}\right)_{t \geqslant 0}$ is a PDMP with characteristics $\left(\varphi^{\otimes}, \lambda^{(1)}, \tilde{K}, r_{2}, K_{2}\right)$ with initial distribution $\delta_{(x, y)}$ defined by Construction 2 based on some random variables $\left(E_{j, k}, U_{j, k}\right)_{j \in \llbracket 1,2 \rrbracket, k \in \mathbb{N}}$. We are now able to show that $\left(X_{t}\right)_{t \geqslant 0}$ is distributed according to $\operatorname{PDMP}\left(\varphi, \lambda^{(1)}, Q^{(1)}, \delta_{x}\right)$. Let $\left(\left(X_{i}^{\prime}, Y_{i}^{\prime}\right), \tilde{S}_{i}, \tilde{I}_{i}\right)_{i \in \mathbb{N}}$ be the embedded chain associated with $\left(X_{t}, Y_{t}\right)_{t \geqslant 0}$. Set $R_{0}=0$, $J_{0}=0$ and, for $k \in \mathbb{N}$,

$$
R_{k+1}=\inf \left\{\tilde{S}_{i}>R_{k}: i \in \mathbb{N}, \tilde{I}_{i}=1\right\}, \quad J_{k+1}=\inf \left\{i>J_{k}: \tilde{I}_{i}=1\right\} .
$$

Note that $R_{k}=S_{J_{k}}$ if $J_{k}<+\infty$ and the process being non-explosive, $\sup _{n \in \mathbb{N}} \tilde{S}_{n}=+\infty$, so that $\sup _{n \in \mathbb{N}} R_{n}=+\infty$. For all $k \in \mathbb{N}$, set $\bar{X}_{k}=X_{R_{k}}$ if $R_{k}<\infty$ and $\bar{X}_{k}=\infty$ otherwise. For $k \in \mathbb{N}$, on $\left\{R_{k}<+\infty\right\}$, by definition of $K_{2}$ and $\varphi^{\otimes}$, an easy induction 
using implies that for any $j \in \llbracket J_{k}, J_{k+1}-1 \rrbracket, t \in\left[S_{j}, S_{j+1}\right), X_{t}=\varphi_{S_{J_{j}}, t}\left(\bar{X}_{S_{J_{j}}}\right)=$ $\varphi_{R_{k}, t}\left(\bar{X}_{k}\right)$, and

$$
H_{1, j+1}=H_{j, 1}-\int_{S_{j}}^{S_{j+1}} \lambda^{(1)}\left(s, \varphi_{S_{j}, s}\left(X_{S_{j}}\right)\right) \mathrm{d} s=E_{k, 1}-\int_{R_{k}}^{S_{j+1}} \lambda^{(1)}\left(s, \varphi_{R_{k}, s}\left(\bar{X}_{k}\right)\right) \mathrm{d} s .
$$

Therefore for all $k \in \mathbb{N}$ such that $R_{k}<\infty$ and all $t \in\left[R_{k}, R_{k+1}\right), X_{t}=\varphi_{R_{k}, t}\left(\bar{X}_{k}\right)$ and

$$
R_{k+1}=\inf \left\{t \geqslant R_{k}: E_{1, k+1}<\int_{R_{k}}^{t} \lambda^{(1)}\left(s, \varphi_{R_{k}, s}\left(\bar{X}_{k}\right)\right) \mathrm{d} s\right\},
$$

Finally, if $R_{k+1}<\infty$, denoting by $\mathbf{G}_{1}, \mathbf{G}_{2}$ the representation of $\tilde{K}$ and $K_{2}$ respectively, used in the construction of the process, for any $j \in \llbracket J_{k}, J_{k+1}-2 \rrbracket$

$$
\begin{aligned}
\left(X_{S_{j+1}}, Y_{S_{j+1}}\right) & =\mathbf{G}_{2}\left(S_{j+1},\left(\varphi_{R_{k}, S_{j+1}}\left(\bar{X}_{k}\right), Y_{S_{j}}\right), U_{2, j+1}\right) \\
\left(X_{R_{k+1}}, Y_{R_{k+1}}\right) & =\mathbf{G}_{1}\left(R_{k+1},\left(\varphi_{R_{k}, R_{k+1}}\left(\bar{X}_{k}\right), Y_{S_{J_{k+1}-1}}\right), U_{1, k+1}\right) .
\end{aligned}
$$

It follows that $U_{1, k+1}$ and $E_{1, k+1}$ are independent of $Y_{S_{J_{k+1}-1}}$ and $\mathcal{F}_{k}$ where $\left(\mathcal{F}_{\tilde{k}}\right)_{\tilde{k} \in \mathbb{N}}$ is the filtration associated with $\left(\bar{X}_{k}, R_{k}\right)_{k \in \mathbb{N}}$. Using that $E_{1, k+1}$ and $U_{1, k+1}$ are independent, (25) and (26) yield, for all $k \in \mathbb{N}, t \geqslant R_{k}$ and $\mathrm{A} \in \mathcal{B}(\mathrm{M})$,

$$
\begin{aligned}
\mathbb{P}\left(\bar{X}_{k+1} \in \mathrm{A}, R_{k+1} \leqslant t \mid\right. & \left.\mathcal{F}_{k}\right) \\
=\mathbb{1}_{\mathrm{M}}\left(\bar{X}_{k}\right) \int_{R_{k}}^{t} \mathbb{P}\left(\mathbf{G}_{1}(\right. & \left.\left.\left.s,\left(\bar{X}_{k+1}, Y_{S_{J_{k+1}-1}}\right)\right), U_{1, k+1}\right) \in \mathrm{A} \mid \mathcal{F}_{k} \vee \sigma\left(Y_{S_{J_{k+1}-1}}\right)\right) \\
& \times \lambda^{(1)}\left(s, \varphi_{R_{k}, s}\left(\bar{X}_{k}\right)\right) \exp \left\{-\int_{R_{k}}^{s} \lambda^{(1)}\left(u, \varphi_{R_{k}, u}\left(\bar{X}_{k}\right)\right) \mathrm{d} u\right\} \mathrm{d} s .
\end{aligned}
$$

Now, for all $k \in \mathbb{N}, t \geqslant 0,(x, y) \in \mathrm{M}^{2}$ and $\mathrm{A} \in \mathcal{B}(\mathrm{M})$,

$$
\begin{aligned}
\lambda^{(1)}(t, x) & \mathbb{P}\left(\mathbf{G}_{1}\left(t,(x, y), U_{1, k+1}\right) \in \mathrm{A} \times \mathrm{M}\right) \\
& =r_{0}(t,(x, y)) K_{0}(t,(x, y), \mathrm{A} \times \mathrm{M})+r_{1}(t,(x, y)) K_{1}(t,(x, y), \mathrm{A} \times \mathrm{M}) \\
& =\left(r_{0}(t,(x, y))+r_{1}(t,(x, y))\right) Q^{(1)}(t, x, \mathrm{~A})=\lambda^{(1)}(t, x) Q^{(1)}(t, x, \mathrm{~A}) .
\end{aligned}
$$

for all $k \in \mathbb{N}, t \geqslant R_{k}$ and $\mathrm{A} \in \mathcal{B}(\mathrm{M})$, implying

$$
\begin{aligned}
& \mathbb{P}\left(\bar{X}_{k+1} \in \mathrm{A}, R_{k+1} \leqslant t \mid \mathcal{F}_{k}\right) \\
& =\mathbb{1}_{\mathrm{M}}\left(\bar{X}_{k}\right) \int_{R_{k}}^{t} Q^{(1)}(s, x, \mathrm{~A}) \lambda^{(1)}\left(s, \varphi_{R_{k}, s}\left(\bar{X}_{k}\right)\right) \exp \left\{-\int_{R_{k}}^{s} \lambda^{(1)}\left(u, \varphi_{R_{k}, u}\left(\bar{X}_{k}\right)\right) \mathrm{d} u\right\} \mathrm{d} s .
\end{aligned}
$$

As a consequence, $\left(\bar{X}_{k}, R_{k}\right)_{k \in \mathbb{N}}$ is the embedded chain corresponding to a PDMP with characteristics $\left(\varphi, \lambda^{(1)}, Q^{(1)}\right)$. The fact that $X_{t}=\varphi_{R_{k}, t}\left(\bar{X}_{k}\right)$ for all $k \in \mathbb{N}$ such that $R_{k}<\infty$ and all $t \in\left[R_{k}, R_{k+1}\right)$ concludes the proof.

Proof of Theorem 11. (A) We first consider the case where (18) holds. The proof is divided in two main steps. In the first one, we assume that $\lambda^{(1)}$ and $\lambda^{(2)}$ are uniformly bounded and the second one is the extension of the first result in the case where the jump rates are not bounded. 
(1) Assume that $\left\|\lambda^{(i)}\right\|_{\infty} \leqslant M$, for $i=1,2$ and some $M>0$. Let $\left(P_{s, t}^{(1)}\right)_{t \geqslant s \geqslant 0}$ and $\left(P_{s, t}^{(2)}\right)_{t \geqslant s \geqslant 0}$ be two PDMPs semigroups with characteristics $\left(\varphi, Q^{(1)}, \lambda^{(1)}\right)$ and $\left(\varphi, Q^{(2)}, \lambda^{(2)}\right)$ respectively. Since $\lambda^{(1)}$ and $\lambda^{(2)}$ are uniformly bounded, the synchronous characteristics $\left(\varphi,\left(r_{i}, K_{i}\right)_{i \in \llbracket 0,2 \rrbracket}\right)$, where $\left(r_{i}, K_{i}\right)_{i \in \llbracket 0,2 \rrbracket}$ is defined in (23) and (24), are non explosive. From Lemma 13, the synchronous coupling $\left(X_{t}, Y_{t}\right)_{t \geqslant 0}$ defined above is a Markov coupling between these two semigroups. Then, by characterisation of the total variation distance for any $x \in \mathrm{M}$ and $t \geqslant 0$,

$$
\left\|\delta_{x} P_{0, t}^{(1)}-\delta_{x} P_{0, t}^{(2)}\right\|_{\mathrm{TV}} \leqslant 2 \mathbb{P}\left(X_{t}=Y_{t}\right) \leqslant 2 \mathbb{P}\left(X_{s}=Y_{s} \text { for any } s \in[0, t]\right),
$$

where $\left(X_{t}, Y_{t}\right)_{t \geqslant 0}$ has distribution $\operatorname{PDMP}\left(\varphi^{\otimes},\left(r_{i}, K_{i}\right)_{i \in \llbracket 0,2 \rrbracket}, \delta_{x}^{\otimes 2}\right)$. However, to do so, we consider a process $\left(X_{t}, Y_{t}\right)_{t \geqslant 0}$ based on different characteristics from $\left(\varphi^{\otimes},\left(r_{i}, K_{i}\right)_{i \in \llbracket 0,2 \rrbracket}\right)$ but still having the expected distribution using Theorem 5 .

Define the Markov kernels, for all $(x, y) \in \mathrm{M}^{2}$ and $(\mathrm{A}, \mathrm{B}) \in \mathcal{B}(\mathrm{M})^{2}$, dropping the subscript $\mathrm{M}$ for the diagonal $\Delta$

$$
\begin{aligned}
& K_{0, \Delta}(t,(x, y), \mathrm{A} \times \mathrm{B})= \begin{cases}\frac{K_{0}(t,(x, y), \mathrm{A} \times \mathrm{B} \cap \Delta)}{K_{0}(t,(x, y), \Delta)} & \text { if } K_{0}(t,(x, y), \Delta) \neq 0 \\
\left.\delta_{(x, y)} \mathrm{A} \times \mathrm{B}\right) & \text { otherwise },\end{cases} \\
& K_{0, \neq}(t,(x, y), \mathrm{A} \times \mathrm{B})= \begin{cases}\frac{K_{0}\left(t,(x, y), \mathrm{A} \times \mathrm{B} \cap \Delta^{\mathrm{c}}\right)}{K_{0}\left(t,(x, y), \Delta^{\mathrm{c}}\right)} & \text { if } K_{0}\left(t,(x, y), \Delta^{\mathrm{c}}\right) \neq 0 \\
\left.\delta_{(x, y)} \mathrm{A} \times \mathrm{B}\right) & \text { otherwise, }\end{cases} \\
& K_{1, \Delta}(t,(x, y), \mathrm{A} \times \mathrm{B})= \begin{cases}\frac{K_{1}(t,(x, y),(\mathrm{A} \cap\{y\}) \times \mathrm{B})}{K_{1}(t,(x, y),\{y\} \times \mathrm{M})} & \text { if } K_{1}(t,(x, y),\{y\} \times \mathrm{M}) \neq 0 \\
\delta_{(x, y)}(\mathrm{A} \times \mathrm{B}) & \text { otherwise, }\end{cases} \\
& K_{1, \neq}(t,(x, y), \mathrm{A} \times \mathrm{B})= \begin{cases}\frac{K_{1}(t,(x, y),(\mathrm{A} \backslash\{y\}) \times \mathrm{M})}{K_{1}(t,(x, y),(\mathrm{M} \backslash\{y\}) \times \mathrm{M})} & \text { if } K_{1}(t,(x, y),(\mathrm{M} \backslash\{y\}) \times \mathrm{M}) \neq 0 \\
\delta_{(x, y)}(\mathrm{A} \times \mathrm{B}) & \text { otherwise, }\end{cases} \\
& K_{2, \Delta}(t,(x, y), \mathrm{A} \times \mathrm{B})= \begin{cases}\frac{K_{2}(t,(x, y), \mathrm{A} \times(\mathrm{B} \cap\{x\}))}{K_{2}(t,(x, y), \mathrm{M} \times\{x\})} & \text { if } K_{2}(t,(x, y), \mathrm{M} \times\{x\}) \neq 0 \\
\delta_{(x, y)}(\mathrm{A} \times \mathrm{B}) & \text { otherwise },\end{cases} \\
& K_{2, \neq}(t,(x, y), \mathrm{A} \times \mathrm{B})= \begin{cases}\frac{K_{2}(t,(x, y), \mathrm{A} \times(\mathrm{B} \backslash\{x\}))}{K_{2}(t,(x, y), \mathrm{M} \times(\mathrm{M} \backslash\{x\}))} & \text { if } K_{2}(t,(x, y), \mathrm{M} \times(\mathrm{M} \backslash\{x\})) \neq 0 \\
\delta_{(x, y)}(\mathrm{A} \times \mathrm{B}) & \text { otherwise } .\end{cases}
\end{aligned}
$$

Define also the rate jumps for all $(x, y) \in \mathrm{M}^{2}$ by

$$
\begin{aligned}
& r_{0, \Delta}(t,(x, y))=K_{0}(t,(x, y), \Delta) r_{0}(x, y), r_{0, \neq}(t, x, y)=K_{0}\left(t,(x, y), \Delta^{\mathrm{c}}\right) r_{0}(x, y), \\
& r_{1, \Delta}(t,(x, y))=K_{1}(t,(x, y),\{y\} \times \mathrm{M}) r_{1}(t,(x, y)), r_{1, \neq}(t,,(x, y))=K_{1}\left(t,(x, y),\{y\}^{\mathrm{c}} \times \mathrm{M}\right) r_{1}(t,(x, y)), \\
& r_{2, \Delta}(t,,(x, y))=K_{2}(t,(x, y), \mathrm{M} \times\{x\}) r_{2}(t,(x, y)), r_{2, \neq}(t,(x, y))=K_{2}\left(t,(x, y), \mathrm{M} \times\{x\}^{\mathrm{c}}\right) r_{2}(t,(x, y)) .
\end{aligned}
$$

By Theorem 5, for any initial distribution $\mu_{0}$ on $\mathrm{M}^{2}$,

$$
\operatorname{PDMP}\left(\varphi^{\otimes},\left(r_{i}, K_{i}\right)_{i \in \llbracket 0,2 \rrbracket}, \mu_{0}\right)=\operatorname{PDMP}\left(\varphi^{\otimes},\left(r_{i, \circ}, K_{i, \circ}\right)_{i \in \llbracket 0,2 \rrbracket, \circ \in\{\Delta, \neq\}}, \mu_{0}\right) .
$$


Let $\left(X_{t}, Y_{t}\right)_{t \geqslant 0}$ be a PDMP associated with the characteristics $\left(\varphi,\left(r_{i, \circ}, K_{i, \circ}\right)_{i \in \llbracket 0,2 \rrbracket, \circ \in\{\Delta, \neq\}}\right)$ with initial distribution $\delta_{x}^{\otimes 2}, x \in \mathrm{M}$, and let $\left(S_{n}^{\neq, i}\right)_{n \in \mathbb{N}, i \in \llbracket 0,2 \rrbracket}$ be the jump times associated with the jump rates $r_{0, \neq}, r_{1, \neq}, r_{2, \neq}$ respectively. By Lemma 13 since $\left(X_{t}, Y_{t}\right)_{t \geqslant 0}$ is non-explosive and Proposition 4 , we get for all $t \geqslant 0$

$$
\begin{aligned}
& \mathbb{P}\left(X_{t} \neq Y_{t}\right) \leqslant 1-\mathbb{P}\left(\min _{i \in \llbracket 0,2 \rrbracket} S_{1}^{\neq, i} \geqslant t, X_{s}=Y_{s} \text { for all } s \in[0, t]\right) \\
& \leqslant 1-\mathbb{E}\left[\exp \left[-\int_{0}^{t}\left\{r_{0, \neq}\left(s,\left(\bar{X}_{s}, \bar{X}_{s}\right)\right)+r_{1, \neq}\left(s,\left(\bar{X}_{s}, \bar{X}_{s}\right)\right)+r_{2, \neq}\left(s,\left(\bar{X}_{s}, \bar{X}_{s}\right)\right)\right\} \mathrm{d} s\right]\right],
\end{aligned}
$$

where $\left(\bar{X}_{s}, \bar{X}_{s}\right)_{s \geqslant 0}$ is a PDMP with characteristics $\left(\tilde{\varphi},\left(r_{i, \Delta}, K_{i, \Delta}\right)_{i \in \llbracket 0,2 \rrbracket}\right)$ starting at $(x, x)$. This result concludes the proof since by definition, (23), (24) and (18), for all $y \in \mathrm{M}$ and $s \geqslant 0$, we have

$$
r_{0, \neq}(s,(y, y))+r_{1, \neq}(s,(y, y))+r_{2, \neq}(s,(y, y)) \leqslant g(s) .
$$

(2) In the case where $\lambda^{(1)}$ and $\lambda^{(2)}$ are not uniformly bounded, consider for all $M>0$ the two semi-groups $\left(P_{s, t}^{(1), M}\right)_{t \geqslant s \geqslant 0}$ and $\left(P_{s, t}^{(2), M}\right)_{t \geqslant s \geqslant 0}$ associated with the characteristics $\left(\varphi, \lambda^{(1)} \wedge M, Q^{(1)}\right)$ and $\left(\varphi, \lambda^{(2)} \wedge M, Q^{(2)}\right)$ respectively. Then for all $M>0$ the triangle inequality yields

$\left\|\delta_{x} P_{0, t}^{(1)}-\delta_{x} P_{0, t}^{(2)}\right\|_{\mathrm{TV}} \leqslant\left\|\delta_{x} P_{0, t}^{(1)}-\delta_{x} P_{0, t}^{(1), M}\right\|_{\mathrm{TV}}+\left\|\delta_{x} P_{0, t}^{(1), M}-\delta_{x} P_{0, t}^{(2), M}\right\|_{\mathrm{TV}}+\left\|\delta_{x} P_{0, t}^{(2), M}-\delta_{x} P_{0, t}^{(2)}\right\|_{\mathrm{TV}}$.

Using Proposition 8 and the assumption that the semi-groups we consider are nonexplosive,

$$
\left\|\delta_{x} P_{0, t}^{(1)}-\delta_{x} P_{0, t}^{(2)}\right\|_{\mathrm{TV}} \leqslant \limsup _{M \rightarrow+\infty}\left\|\delta_{x} P_{0, t}^{(1), M}-\delta_{x} P_{0, t}^{(2), M}\right\|_{\mathrm{TV}} .
$$

On the other hand, by the first part of the proof for all $M>0$,

$$
\left\|\delta_{x} P_{0, t}^{(1), M}-\delta_{x} P_{0, t}^{(2), M}\right\|_{\mathrm{TV}} \leqslant 2\left\{1-\exp \left(-\int_{0}^{t} g(s) \mathrm{d} s\right)\right\},
$$

where $g$ satisfies (18) since for all $t \in \mathbb{R}_{+}$and $M>0$,

$$
\begin{aligned}
g(t) \geqslant \sup _{\substack{x \in \mathbb{M}, \mathrm{A} \in \mathcal{B}(\mathrm{M})}}\left\{M \wedge \lambda^{(1)}(t, x) \wedge \lambda^{(2)}(t, x)\left|Q^{(1)}(t, x, \mathrm{~A})-Q^{(2)}(t, x, \mathrm{~A})\right|\right\} \\
\\
\quad+\sup _{x \in \mathbb{M}}\left|M \wedge \lambda^{(1)}(t, x)-M \wedge \lambda^{(2)}(t, x)\right| .
\end{aligned}
$$

Combining (28) and (29) concludes the proof.

(B) Let us finish the proof by assuming (19) and showing that the conclusion of Theorem 11 still holds. Indeed by Theorem 5, for all initial distribution $\mu_{0} \in \mathcal{P}(\mathrm{M})$, $\operatorname{PDMP}\left(\varphi, Q^{(1)}, \lambda^{(1)}, \mu_{0}\right)=\operatorname{PDMP}\left(\varphi, \tilde{Q}^{(1)}, \tilde{\lambda}^{(1)}, \mu_{0}\right)$ and $\operatorname{PDMP}\left(\varphi, Q^{(2)}, \lambda^{(2)}, \mu_{0}\right)=\operatorname{PDMP}\left(\varphi, \tilde{Q}^{(2)}, \tilde{\lambda}^{(2)}, \mu_{0}\right)$ where $\tilde{\lambda}^{(1)}=\tilde{\lambda}^{(2)}=\lambda^{(1)} \vee \lambda^{(2)}$ and for $t \in \mathbb{R}_{+}, x \in \mathrm{M}, \mathrm{A} \in \mathcal{B}(\mathrm{M})$ and $i=1,2$

$$
\tilde{Q}^{i}(t, x, \mathrm{~A})=\frac{\lambda^{(i)}(t, x)}{\lambda^{(1)}(t, x) \vee \lambda^{(2)}(t, x)} Q^{i}(t, x, \mathrm{~A})+\left(1-\frac{\lambda^{(i)}(t, x)}{\lambda^{(1)}(t, x) \vee \lambda^{(2)}(t, x)}\right) \delta_{x}(\mathrm{~A}) .
$$

Therefore, $\left(P_{s, t}^{(1)}\right)_{t \geqslant s \geqslant 0}$ and $\left(P_{s, t}^{(2)}\right)_{t \geqslant s \geqslant 0}$ are also associated with $\left(\varphi, \tilde{Q}^{(1)}, \tilde{\lambda}^{(1)}\right)$ and $\left(\varphi, \tilde{Q}^{(2)}, \tilde{\lambda}^{(2)}\right)$. Applying the case where $g$ is given by Equation (18) to these characteristics concludes. 


\section{Generator}

From this section, only homogeneous processes are considered. Nevertheless, some results below can be applied to inhomogeneous PDMP since if $\left(X_{t}\right)_{t \geqslant 0}$ is such a process on $\mathrm{M}$ with characteristics $(\varphi, Q, \lambda)$, then the process $\left(X_{t}, t\right)_{t \geqslant 0}$ is a homogeneous PDMP on $\left(\mathrm{M} \times \mathbb{R}_{+}, \mathcal{B}\left(\mathrm{M} \times \mathbb{R}_{+}\right)\right)$with characteristics $(\bar{\varphi}, \bar{Q}, \lambda)$ defined for all $t, s \in \mathbb{R}_{+}, x \in \mathrm{M}$ and $\mathrm{A} \in \mathcal{B}\left(\mathrm{M} \times \mathbb{R}_{+}\right)$by

$$
\bar{\varphi}_{s}((x, t))=\left(\varphi_{t, t+s}(x), t+s\right), \quad \bar{Q}((x, t), \mathrm{A})=\int_{\mathrm{M} \times \mathbb{R}_{+}} \mathbb{1}_{\mathrm{A}}((y, u)) Q(x, \mathrm{~d} y) \otimes \delta_{t}(\mathrm{~d} u) .
$$

This section is devoted to the introduction of the strong and extended generators of a non-explosive PDMP, which will be a central tool for the study of invariant measures (see Theorem 21 and Corollary 22 below).

Consider a homogeneous PDMP semigroup $\left(P_{t}\right)_{t \geqslant 0}$ with non-explosive characteristics $(\varphi, \lambda, Q)$. Note that $\left(P_{t}\right)_{t \geqslant 0}$ is a contraction semigroup on $\mathrm{B}(\mathrm{M})$, i.e. for all $s, t \in \mathbb{R}_{+}$, $P_{s+t}=P_{t} P_{s}$ and for all function $f \in \mathrm{B}(\mathrm{M}),\left\|P_{t} f\right\|_{\infty} \leqslant\|f\|_{\infty}$. In addition, define the subset $\mathrm{B}_{0}(\mathrm{M}) \subset \mathrm{B}(\mathrm{M})$ by

$$
\mathrm{B}_{0}(\mathrm{M})=\left\{f \in \mathrm{B}(\mathrm{M}): \lim _{t \rightarrow 0}\left\|P_{t} f-f\right\|_{\infty}=0\right\} .
$$

By $\left[9\right.$, p.28-29], $\mathrm{B}_{0}(\mathrm{M})$ is a closed subspace of $\mathrm{B}(\mathrm{M})$ and a Banach space for the uniform norm. Then by definition, $\left(P_{t}\right)_{t \geqslant 0}$ is a strongly continuous semigroup on $\mathrm{B}_{0}(\mathrm{M})$, i.e. for all $f \in \mathrm{B}_{0}(\mathrm{M}), \lim _{t \rightarrow 0}\left\|P_{t} f-f\right\|_{\infty}=0$.

Define $(\overline{\mathcal{A}}, \mathrm{D}(\overline{\mathcal{A}}))$ the strong generator of $\left(P_{t}\right)_{t \geqslant 0}$ by

$$
\begin{aligned}
\mathrm{D}(\overline{\mathcal{A}}) & =\left\{f \in \mathrm{B}_{0}(\mathrm{M}): \text { there exists } g: \mathrm{M} \rightarrow \mathbb{R} \lim _{t \rightarrow 0}\left\|t^{-1}\left(P_{t} f-f\right)-g\right\|_{\infty}=0\right\}, \\
\overline{\mathcal{A}} f & =g \text { satisfying } \lim _{t \rightarrow 0}\left\|t^{-1}\left(P_{t} f-f\right)-g\right\|_{\infty}=0 \text { for all } f \in \mathrm{D}(\overline{\mathcal{A}}) .
\end{aligned}
$$

A subset $\mathrm{D} \subset \mathrm{D}(\overline{\mathcal{A}})$ is a core of $(\overline{\mathcal{A}}, \mathrm{D}(\overline{\mathcal{A}}))$ if the closure of the restriction of $\overline{\mathcal{A}}$ to $\mathrm{D}$ is equal to $(\overline{\mathcal{A}}, \mathrm{D}(\overline{\mathcal{A}}))$. The strong generator of $\left(P_{t}\right)_{t \geqslant 0}$ is a common tool to show that a probability measure on $(\mathrm{M}, \mathrm{B}(\mathrm{M}))$ is invariant for $\left(P_{t}\right)_{t \geqslant 0}$. Indeed by [13, Proposition 9.2], $\mu$ is invariant measure for $\left(P_{t}\right)_{t \geqslant 0}$ if only if for all $f \in \mathrm{D}$, where $\mathrm{D}$ is a core for $(\overline{\mathcal{A}}, \mathrm{D}(\overline{\mathcal{A}}))$, $\int_{\mathrm{M}} \overline{\mathcal{A}} f(x) \mu(\mathrm{d} x)=0$. Therefore, the strong generator $(\overline{\mathcal{A}}, \mathrm{D}(\overline{\mathcal{A}}))$ is an essential tool to study $\left(P_{t}\right)_{t \geqslant 0}$. Unfortunately, characterizing the domain $\mathrm{D}(\overline{\mathcal{A}})$ is not possible in many cases of interest. In addition, while it would be possible to only use a core of $(\overline{\mathcal{A}}, \mathrm{D}(\overline{\mathcal{A}}))$, there are very few results giving such a subset for PDMPs contrary to diffusion processes (see e.g. [13, Chapter 8]). However, we will see in Section 9 that for a class of PDMPs, to show that a probability measure $\mu$ is invariant, it is sufficient to show that for all $f \in \mathrm{C}_{c}^{1}(\mathrm{M}), \int_{\mathrm{M}} \mathcal{A} f(x) \mathrm{d} \mu(x)=0$, where $(\mathcal{A}, \mathrm{D}(\mathcal{A}))$ is the extended generator of $\left(P_{t}\right)_{t \geqslant 0}$, defined as follows.

For $x \in \mathrm{M}$, denote $\mathbb{P}_{x}$ the distribution $\operatorname{PDMP}\left(\varphi, \lambda, Q, \delta_{x}\right)$ on $\mathrm{D}\left(\mathbb{R}_{+}, \mathrm{M}\right)$ and $\mathbb{E}_{x}$ the corresponding expectation. Let $\left(\bar{X}_{t}\right)_{t \geqslant 0}$ be the canonical process on $\mathrm{D}\left(\mathbb{R}_{+}, \mathrm{M}\right)$, defined by $\bar{X}_{t}(\omega)=\omega_{t}$ for all $\omega \in \mathrm{D}\left(\mathbb{R}_{+}, \mathrm{M}\right)$, and let $\left(\mathcal{F}_{t}\right)_{t \geqslant 0}$ be its associated filtration. Let 
$\bar{S}_{0}=0$ and, for $k \in \mathbb{N}, \bar{S}_{k+1}=\inf \left\{t>\bar{S}_{k}: \bar{X}_{t} \neq \varphi_{t-\bar{S}_{k}}\left(\bar{X}_{\bar{S}_{k}}\right)\right\}$ be its true jump times. Define for all $t \in \mathbb{R}_{+}, \bar{N}_{t}=\sum_{k \in \mathbb{N}^{*}} \mathbb{1}_{[0, t]}\left(\bar{S}_{k}\right)$ and consider the following assumption

A 1. For all $x \in \mathrm{M}$ and $t \in \mathbb{R}_{+}, \mathbb{E}_{x}\left[\bar{N}_{t}\right]<+\infty$.

For all $t \geqslant 0$ and for all measurable functions $f, g: \mathrm{M} \rightarrow \mathbb{R}$, such that, for all $x \in \mathrm{M}$, $s \mapsto g\left(\bar{X}_{s}\right)$ is $\mathbb{P}_{x}$-almost surely locally integrable, denote

$$
M_{t}^{f, g}=f\left(\bar{X}_{t}\right)-f\left(\bar{X}_{0}\right)-\int_{0}^{t} g\left(\bar{X}_{s}\right) \mathrm{d} s .
$$

The (extended) generator and its domain $(\mathcal{A}, \mathrm{D}(\mathcal{A}))$ associated with the semi-group $\left(P_{t}\right)_{t \geqslant 0}$ are defined as follows: $f \in \mathrm{D}(\mathcal{A})$ if there exists a measurable function $g: \mathrm{M} \rightarrow \mathbb{R}$ such that $\left(M_{t}^{f, g}\right)_{t \geqslant 0}$ is a local martingale under $\mathbb{P}_{x}$ for all $x \in \mathrm{M}$ and, for such a function, $\mathcal{A} f=g$. Despite its very formal definition, $(\mathcal{A}, \mathrm{D}(\mathcal{A}))$ associated with $\left(P_{t}\right)_{t \geqslant 0}$ can be easily described. Indeed, under $\mathbf{A} 1$, [9, Theorem 26.14] shows that $\mathrm{D}(\mathcal{A})=\mathrm{E}_{1} \cap \mathrm{E}_{2}$ where

$$
\mathrm{E}_{1}=\left\{f \in \mathbb{M}(\mathrm{M}): t \mapsto f\left(\varphi_{t}(x)\right) \text { is absolutely continuous on } \mathbb{R}_{+} \text {for all } x \in \mathrm{M}\right\},
$$

and $\mathrm{E}_{2}$ is the set of measurable functions $f: M \rightarrow \mathbb{R}$ such that there exists an increasing sequence of $\left(\mathcal{F}_{t}\right)_{t \geqslant 0}$-stopping time $\left(\sigma_{n}\right)_{n \geqslant 0}$, such that for all $x \in \mathrm{M}, \lim _{n \rightarrow+\infty} \sigma_{n}=+\infty$ $\mathbb{P}_{x}$-almost surely and for all $n \in \mathbb{N}$,

$$
\mathbb{E}_{x}\left[\sum_{k=0}^{+\infty} \mathbb{1}_{\left\{\bar{S}_{k+1} \leqslant \sigma_{n}\right\}}\left|f\left(\bar{X}_{\bar{S}_{k+1}}\right)-f\left(\varphi_{\bar{S}_{k+1}-\bar{S}_{k}}\left(\bar{X}_{\bar{S}_{k}}\right)\right)\right|\right]<+\infty .
$$

Then, for all $f \in \mathrm{D}(\mathcal{A})$ and $x \in \mathrm{M}$,

$$
\mathcal{A} f(x)=D_{\varphi} f(x)+\lambda(x)(Q f(x)-f(x)),
$$

where

$$
D_{\varphi} f(x)= \begin{cases}\lim _{t \downarrow 0} t^{-1}\left\{f\left(\varphi_{t}(x)\right)-f(x)\right\}, & \text { if this limit exists } \\ 0 & \text { otherwise } .\end{cases}
$$

In fact, in [9, Theorem 26.14], $Q$ is required to satisfy $Q(x,\{x\})=0$ for all $x \in \mathrm{M}$, but if it is not the case, from Theorem 5 , we can apply this result with the minimal jump rate associated to $(\lambda, Q)$ such as introduced in Section 4.

Note that $\mathrm{D}(\overline{\mathcal{A}}) \subset \mathrm{D}(\mathcal{A})$ and for all $f \in \mathrm{D}(\overline{\mathcal{A}}), \mathcal{A} f=\overline{\mathcal{A}} f$, since by [9, Proposition 14.13], for all $f \in \mathrm{D}(\overline{\mathcal{A}}),\left(M_{t}^{f, \overline{\mathcal{A}} f}\right)_{t \geqslant 0}$ is a $\left(\mathcal{F}_{t}\right)_{t \geqslant 0}$-martingale.

In addition, $\mathrm{C}^{1}(\mathrm{M}) \subset \mathrm{D}(\mathcal{A})$ and, if $f \in \mathrm{C}_{c}^{1}(\mathrm{M})$, then $\mathcal{A} f$ is bounded, therefore $\left(M_{t}^{f, \mathcal{A} f}\right)_{t \geqslant 0}$ is a $\left(\mathcal{F}_{t}\right)_{t \geqslant 0}$-martingale. Moreover, since we supposed that $b(x)=\left(\partial_{t}\right)_{t=0} \varphi_{t}(x)$ exists for all $x \in \mathrm{M}$, then $D_{\varphi} f(x)=\langle b(x), \nabla f(x)\rangle$ for all $f \in \mathrm{C}^{1}(\mathrm{M})$ and $x \in \mathrm{M}$. However, we need some conditions on $\lambda$ and $Q$ to show that that $\mathrm{C}_{c}^{1}(\mathrm{M}) \subset \mathrm{D}(\overline{\mathcal{A}})$.

A 2. Let $\left(P_{t}\right)_{t \geqslant 0}$ be a non explosive PDMP semi-group with characteristics $(\varphi, \lambda, Q)$. Assume that for all $T \geqslant 0$, there exists $M \geqslant 0$ such that for all $x \in \mathrm{M}$ and $t \in[0, T]$, $\operatorname{supp}\left\{P_{t}(x, \cdot)\right\} \subset \overline{\mathrm{B}}(x, M)$.

Lemma 14. Assume A2.

(a) For all $f \in \mathrm{C}_{c}(\mathrm{M}), T \in \mathbb{R}_{+}$, there exists a bounded set $\mathrm{A}$ such that $P_{t} f(x)=0$, for all $x \notin \mathrm{A}$ and $t \in[0, T]$. 
(b) Condition $\boldsymbol{A} 1$ is satisfied.

Proof. (a) Let $f \in \mathrm{C}_{c}(\mathrm{M}), T \in \mathbb{R}_{+}$. By assumption, there exist $M_{f}, M_{T} \in \mathbb{R}_{+}$such that $\operatorname{supp}(f) \subset \mathrm{B}\left(x_{0}, M_{f}\right), x_{0} \in \mathrm{M}$, and $\operatorname{supp}\left\{P_{t}(x, \cdot)\right\} \subset \overline{\mathrm{B}}\left(x, M_{T}\right)$ for all $t \in[0, T], x \in \mathrm{M}$. Therefore, we get that, for all $x \notin \overline{\mathrm{B}}\left(x_{0}, M_{T}+M_{f}+1\right)$,

$$
P_{t} f(x)=\int_{\mathrm{M}} \mathbb{1}_{\overline{\mathrm{B}}\left(x, M_{T}\right) \cap \mathrm{B}\left(x_{0}, M_{f}\right)}(y) f(y) P_{t}(x, \mathrm{~d} y)=0 .
$$

Indeed, by construction $\overline{\mathrm{B}}\left(x, M_{T}\right) \cap \mathrm{B}\left(x_{0}, M_{f}\right)=\emptyset$ since by the triangle inequality, $\operatorname{dist}(x, y) \leqslant M_{T}$ implies that $\operatorname{dist}\left(x_{0}, y\right) \geqslant M_{f}+1$.

(b) Let $\left(X_{t}\right)_{t \geqslant 0}$ be a PDMP process with characteristics $(\varphi, \lambda, Q)$ started from $x \in \mathrm{M}$, given by Construction 1 , with jump times $\left(S_{k}\right)_{k \in \mathbb{N}}$. Note that by definition, for all $k \in \mathbb{N}, S_{k} \leqslant \bar{S}_{k}$, where $\left(\bar{S}_{k}\right)_{k \in \mathbb{N}}$ is the true jump times of the process. Therefore, defining $N_{t}=\sum_{k=1}^{+\infty} \mathbb{1}_{[0, t]}\left(S_{k}\right)$, we have for all $t \geqslant 0, \bar{N}_{t} \leqslant N_{t}$, and to show that $\mathbf{A} 1$ holds, it suffices to show that $\mathbb{E}\left[N_{T}\right]<+\infty$ for all $T \in \mathbb{R}_{+}$and $x \in \mathrm{M}$.

Let $T \geqslant 0$ and $M_{T} \geqslant 0$ be such that for all $t \in[0, T]$ and $y \in \mathrm{M}, \operatorname{supp}\left\{P_{t}(y, \cdot)\right\} \subset$ $\mathrm{B}\left(y, M_{T}\right)$. Then, since $\left(X_{t}\right)_{t \geqslant 0}$ is a càdlàg process, almost surely, for all $t \in[0, T]$, $X_{t} \in \overline{\mathrm{B}}\left(x, M_{T}\right)$. Therefore, by (1), for all $k \in \mathbb{N}$, and $t \in[0, T]$,

$$
\mathbb{1}_{[0, t]}\left(S_{k+1}\right)\left(S_{k+1}-S_{k}\right) \geqslant E_{k+1} /\left(1+\|\lambda\|_{\infty, \overline{\mathrm{B}}\left(x, M_{T}\right)}\right) .
$$

Then, for all $t \in[0, T], N_{t}$ is bounded by $\sum_{k=1}^{+\infty} \mathbb{1}_{[0, t]}\left(E_{k+1} /\left(1+\|\lambda\|_{\infty, \mathrm{B}\left(x, M_{T}\right)}\right)\right)$, which is a Poisson process with rate $1+\|\lambda\|_{\infty, \mathrm{B}\left(x, M_{T}\right)}$. Therefore, for all $t \in[0, T], \mathbb{E}\left[N_{t}\right]<+\infty$.

Proposition 15. Assume $\boldsymbol{A} 2$.

(a) If $(t, x) \mapsto \varphi_{t}(x) \in \mathrm{C}^{1}\left(\mathbb{R}_{+} \times \mathrm{M}, \mathrm{M}\right)$, then $\mathrm{C}_{0}(\mathrm{M}) \subset \mathrm{B}_{0}(\mathrm{M})$, where $\mathrm{B}_{0}(\mathrm{M})$ is defined by $(30)$.

(b) If $(t, x) \mapsto \varphi_{t}(x) \in \mathrm{C}^{1}\left(\mathbb{R}_{+} \times \mathrm{M}, \mathrm{M}\right), \lambda \in \mathrm{C}(\mathrm{M})$ and for all $f \in \mathrm{C}_{c}(\mathrm{M}), \lambda Q f \in$ $\mathrm{C}_{c}(\mathrm{M})$, then $\mathrm{C}_{c}^{1}(\mathrm{M}) \subset \mathrm{D}(\overline{\mathcal{A}})$.

Proof. (a) We show that $\mathrm{C}_{c}(\mathrm{M}) \subset \mathrm{B}_{0}(\mathrm{M})$ which is sufficient since the closure of $\mathrm{C}_{c}(\mathrm{M})$ for the uniform norm is $\mathrm{C}_{0}(\mathrm{M})$ and $\mathrm{B}_{0}(\mathrm{M})$ is a Banach space equipped with the topology endowed with this norm. Let $f \in \mathrm{C}_{c}(\mathrm{M})$. By Lemma 14-(a), there exists a compact set $\mathrm{K}$ such that for any $x \notin \mathrm{K}$ and $t \in[0,1], P_{t} f(x)=0$. Therefore we only need to show that $\lim _{t \rightarrow+\infty}\left\|P_{t} f-f\right\|_{\infty, \mathrm{K}}=0$. Let $x \in \mathrm{K}$ and consider $\left(X_{t}\right)_{t \geqslant 0}$ the PDMP process with characteristics $(\varphi, \lambda, Q)$ started from $x$ given by Construction 1, with jump times $\left(S_{k}\right)_{k \in \mathbb{N}}$. Then by definition, we have for any $x \in \mathrm{K}$,

$$
\left|P_{t} f(x)-f(x)\right| \leqslant\|f\|_{\infty} \mathbb{P}\left(S_{1} \leqslant t\right)+\left|f\left(\varphi_{t}(x)\right)-f(x)\right| .
$$

On the other hand, let $M \geqslant 0$ be such that for all $t \in[0,1]$ and $y \in \mathrm{M}, \operatorname{supp}\left\{P_{t}(y, \cdot)\right\} \subset$ $\mathrm{B}(y, M)$, and define $\tilde{\mathbf{K}}=\{y \in \mathbf{M}: \operatorname{dist}(y, \mathbf{K}) \leqslant M\}$, which is bounded since $\mathbf{K}$ is. Therefore, we get by (1) for $k=1$,

$$
\mathbb{1}_{[0, t]}\left(S_{1}\right) S_{1} \geqslant E_{1} /\left(1+\|\lambda\|_{\infty, \overline{\mathrm{B}}(x, M)}\right) \geqslant E_{1} /\left(1+\|\lambda\|_{\infty, \tilde{\mathrm{K}}}\right),
$$


where $\|\lambda\|_{\infty, \tilde{\mathrm{K}}}<+\infty$ because $\lambda$ is assumed to be locally bounded. From this result and (33), it follows that for any $x \in \mathrm{K}$,

$$
\left|P_{t} f(x)-f(x)\right| \leqslant\|f\|_{\infty} \mathbb{P}\left(E_{1} \leqslant\left(1+\|\lambda\|_{\infty, \tilde{\mathrm{K}}}\right) t\right)+\left|f\left(\varphi_{t}(x)\right)-f(x)\right| .
$$

In addition, using that $(t, x) \mapsto \varphi_{t}(x) \in \mathrm{C}^{1}(\mathrm{M})$, there exists $C_{1} \geqslant 0$ such that for all $t \in[0,1]$ and $x \in \mathrm{K}$, $\operatorname{dist}\left(\varphi_{t}(x), x\right) \leqslant t C_{1}$. Then, since $f$ is continuous, it is uniformly continuous on $\left\{y \in \mathrm{M}: \operatorname{dist}(y, \mathrm{~K}) \leqslant C_{1}\right\}$ and therefore for all $\varepsilon>0$, there exists $\eta>0$ such that for all $x \in \mathrm{K}$ and $s \in[0, \eta],\left|f\left(\varphi_{s}(x)\right)-f(x)\right| \leqslant \varepsilon$. Combining this result and (34), we obtain that for any $t \in[0, \eta]$,

$$
\left\|P_{t} f-f\right\|_{\infty, \mathrm{K}} \leqslant\|f\|_{\infty} \mathbb{P}\left(E_{1} \leqslant\left(1+\|\lambda\|_{\infty, \tilde{\mathrm{K}}}\right) t\right)+\varepsilon .
$$

Taking $t, \varepsilon \rightarrow 0$ concludes the proof of (a).

(b) Let $f \in \mathrm{C}_{c}^{1}(\mathrm{M})$. By Lemma 14-(a) and since $\lambda Q f \in \mathrm{C}_{c}(\mathrm{M})$, there exists a compact set $\mathrm{K} \subset \mathrm{M}$, such that for all $x \notin \mathrm{K}, P_{t} f(x)=0$, for all $t \in(0,1], \lambda(x) Q f(x)=0$ and $f(x)=0$. Therefore, for all $t \in(0,1]$,

$$
\left\|t^{-1}\left(P_{t} f(x)-f(x)\right)-\mathcal{A} f(x)\right\|_{\infty}=\left\|t^{-1}\left(P_{t} f(x)-f(x)\right)-\mathcal{A} f(x)\right\|_{\infty, \mathrm{K}} .
$$

As seen above, since $f \in \mathrm{C}_{c}^{1}(\mathrm{M}),\left(M_{t}^{f, \mathcal{A} f}\right)_{t \geqslant 0}$, defined by $(31)$, is a $\left(\mathcal{F}_{t}\right)_{t \geqslant 0}$-martingale. Therefore, for all $x \in \mathrm{M}$,

$$
t^{-1}\left\{P_{t} f(x)-f(x)\right\}-\mathcal{A} f(x)=t^{-1} \mathbb{E}_{x}\left[\int_{0}^{t}\left\{\mathcal{A} f\left(\bar{X}_{s}\right)-\mathcal{A} f(x)\right\} \mathrm{d} s\right] .
$$

Then since $f \in \mathrm{C}_{c}^{1}(\mathrm{M}),(t, x) \mapsto \varphi_{t}(x)$ is continuously differentiable, $\lambda$ is locally bounded and $\lambda Q f$ is bounded, there exists $C_{1} \geqslant 0$ such that for all $t>0$ and $x \in \mathrm{M}$, we have

$$
\left|t^{-1}\left\{P_{t} f(x)-f(x)\right\}-\mathcal{A} f(x)\right| \leqslant C_{1} \mathbb{P}_{x}\left(\bar{S}_{1} \leqslant t\right)+t^{-1} \mathbb{E}_{x}\left[\mathbb{1}_{(t,+\infty)}\left(\bar{S}_{1}\right) \int_{0}^{t}\left\{A_{s}^{1}+A_{s}^{2}+A_{s}^{3}\right\} \mathrm{d} s\right],
$$

where

$$
\begin{aligned}
& A_{s}^{1}=\left\langle\left(\partial_{u}\right)_{u=0}\left\{\varphi_{u}\right\}\left(\varphi_{s}(x)\right), \nabla f\left(\varphi_{s}(x)\right)\right\rangle-\left\langle\left(\partial_{u}\right)_{u=0}\left\{\varphi_{u}\right\}(x), \nabla f(x)\right\rangle \\
& A_{s}^{2}=\lambda\left(\varphi_{s}(x)\right) Q f\left(\varphi_{s}(x)\right)-\lambda(x) Q f(x), A_{s}^{3}=-\lambda\left(\varphi_{s}(x)\right) f\left(\varphi_{s}(x)\right)-\lambda(x) f(x) .
\end{aligned}
$$

Using that $(x, s) \mapsto \varphi_{s}(x)$ is locally bounded on $\mathbb{R}_{+} \times \mathrm{M}$ and $\lambda$ on $\mathrm{M}$, there exists $C_{2}$ such that for all $t \in(0,1]$ and $x \in \mathrm{K}$,

$$
\mathbb{P}_{x}\left(\bar{S}_{1} \leqslant t\right) \leqslant \int_{0}^{t} \mathrm{~d} s \lambda\left(\varphi_{s}(x)\right) \exp \left(-\int_{0}^{s} \mathrm{~d} u \lambda\left(\varphi_{u}(x)\right)\right) \leqslant C_{2} t
$$

In addition, using that $(t, x) \mapsto \varphi_{t}(x) \in \mathrm{C}^{1}(\mathrm{M})$, there exists $C_{3} \geqslant 0$ such that for all $t \in(0,1]$ and $x \in \mathrm{K}$, dist $\left(\varphi_{t}(x), x\right) \leqslant t C_{3}$. Then, since $\left\langle\left(\partial_{u}\right)_{u=0} \varphi_{u}, \nabla f\right\rangle, \lambda Q f$ and $\lambda f$ are continuous, they are uniformly continuous on $\left\{y \in \mathrm{M}: \operatorname{dist}(y, \mathrm{~K}) \leqslant C_{3}\right\}$ and therefore for all $\varepsilon>0$, there exists $\eta>0$ such that for all $x \in \mathrm{K}$ and $s \in[0, \eta],\left|A_{s}^{i}\right| \leqslant \varepsilon, i=1,2,3$. Combining this result and (38) in (37), we get for all $x \in \mathrm{K}, \varepsilon>0$ and $t \in[0, \eta \wedge 1]$,

$$
\left|t^{-1}\left\{P_{t} f(x)-f(x)\right\}-\mathcal{A} f(x)\right| \leqslant C_{1} C_{2} t+3 \varepsilon .
$$


Therefore, by (36) we get for all $\varepsilon>0$,

$$
\limsup _{t \downarrow 0}\left\|t^{-1}\left\{P_{t} f(x)-f(x)\right\}-\mathcal{A} f(x)\right\|_{\infty} \leqslant 3 \varepsilon .
$$

Taking $\varepsilon \rightarrow 0$ concludes the proof.

Let us finish by our running example.

Example - Bouncy Particle Sampler. Consider the BPS process defined in Example 1 and suppose that $\mathbf{Y}$ is bounded. It is then easy to verify that $\mathbf{A} 2$ is verified, and the strong generator is given for all $f \in \mathrm{C}_{c}^{1}(\mathrm{M})$ by

$$
\begin{aligned}
\overline{\mathcal{A}} f(x, y)=\langle y, \nabla f(x, y)\rangle+(\langle y, \nabla U(x)\rangle)_{+}(f(x, & R(x, y))-f(x, y)) \\
& +\lambda_{\mathrm{c}}\left(\int_{\mathrm{Y}} f(x, w) \mathrm{d} \mu_{\mathrm{v}}(w)-f(x, y)\right) .
\end{aligned}
$$

\section{REgularity ESTIMATES FOR PDMP SEMigroups}

After introducing the strong and extended generators of a PDMP in the previous section, we continue to set up the intermediary tools that will eventually lead to a simple criterion for verifying that a probability measure is invariant for a given PDMP (Theorem 21 and Corollary 22 in the next section). In case of Markov semigroups defined through diffusion processes, regularization properties can be established which usually imply that some class of smooth functions is a core for the strong generator of the semigroup under consideration. In this spirit, the main goal of this section is to provide similar results for PDMPs, i.e. we provide conditions upon which some class of smooth functions is a core for the strong generator of a PDMP (see also [2] on that topic). However, compared to diffusion semigroups, we cannot expect using regularization properties.

To do so, we need the following definition.

Definition 16. We say that a homogeneous differential flow $\varphi$ on $\mathrm{M}$ and a homogeneous Markov kernel $Q$ on $\mathrm{M}$ are compactly compatible if for all compact set $\mathrm{K} \subset \mathrm{M}$ and $T \geqslant 0$, there exists a compact set $\tilde{\mathrm{K}} \subset \mathrm{M}$ satisfying: for all $n \in \mathbb{N}^{*},\left(t_{i}\right)_{i \in \llbracket 1, n \rrbracket} \in \mathbb{R}_{+}^{n}, \sum_{i=1}^{n} t_{i} \leqslant T$, there exists a sequence $\left(\mathrm{K}_{i}\right)_{i \in \llbracket 1, n \rrbracket}$ of compact sets of $\mathrm{M}$ such that, setting $\mathrm{K}_{0}=\mathrm{K}$,

(i) for all $i \in \llbracket 1, n \rrbracket, \mathrm{K}_{i}$ only depends on $\left(t_{j}\right)_{j \in \llbracket 1, i \rrbracket}$ and $\cup_{i=0}^{n} \mathrm{~K}_{i} \subset \tilde{\mathrm{K}}$;

(ii) for all $i \in \llbracket 0, n-1 \rrbracket, s_{i+1} \in\left[0, t_{i+1}\right]$ and $s_{n+1} \in\left[0, T-\sum_{j=1}^{n} t_{j}\right]$,

$$
\bigcup_{x \in \mathrm{K}_{i}} \operatorname{supp}\left\{Q\left(\varphi_{t_{i+1}}(x), \cdot\right)\right\} \subset \mathrm{K}_{i+1}, \quad \varphi_{s_{i+1}}\left(\mathrm{~K}_{i}\right) \subset \tilde{\mathrm{K}}, \quad \varphi_{s_{n+1}}\left(\mathrm{~K}_{n}\right) \subset \tilde{\mathrm{K}}
$$

Note that by definition, if $\varphi$ and $Q$ are compactly compatible and the PDMP semigroup with characteristics $(\varphi, \lambda, Q)$ is non explosive, for all $T \geqslant 0$ and all compact set $\mathrm{K} \subset \mathrm{M}$, there exists a compact set $\tilde{\mathrm{K}} \subset \mathrm{M}$, such that $\mathbb{P}\left(X_{t} \in \tilde{\mathrm{K}}\right.$, for all $\left.t \in[0, T]\right)=1$, where $\left(X_{t}\right)_{t \geqslant 0}$ is a PDMP process with characteristics $(\varphi, \lambda, Q)$ and starting from $X_{0} \in \mathrm{K}$.

A 3. The homogeneous characteristics $(\varphi, \lambda, Q)$ satisfy

(i) the flow $\varphi$ and the Markov kernel $Q$ are compactly compatible; 
(ii) $\lambda \in \mathrm{C}^{1}(\mathrm{M})$ and for all $f \in \mathrm{C}^{1}(\mathrm{M}), \lambda Q f \in \mathrm{C}^{1}(\mathrm{M})$ and there exists a locally bounded function $\Psi: \mathrm{M} \rightarrow \mathbb{R}_{+}$such that for all $x \in \mathrm{K}$,

$$
\|\nabla(\lambda Q f)(x)\| \leqslant\|\Psi\|_{\infty, \mathrm{K}} \sup \{|f(y)|+\|\nabla f(y)\|: y \in \operatorname{supp}\{Q(x, \cdot)\}\} ;
$$

(iii) $(t, x) \mapsto \varphi_{t}(x) \in \mathrm{C}^{1}\left(\mathbb{R}_{+} \times \mathrm{M}\right)$ and for all compact $\mathrm{K} \subset \mathrm{M}$ and $t \geqslant 0$,

$$
\sup \left\{\left\|\nabla \varphi_{s}(x)\right\|: s \in[0, t], x \in \mathrm{K}\right\}<+\infty .
$$

Theorem 17. Let $\left(P_{t}\right)_{t \geqslant 0}$ be a non explosive PDMP semigroup on $\mathrm{M}$ with corresponding characteristics $(\varphi, \lambda, Q)$ satisfying $\boldsymbol{A}$ 3. Then for all $f \in \mathrm{C}^{i}(\mathrm{M}), i \in\{0,1\}, T \in \mathbb{R}_{+}$, $P_{T} f \in \mathrm{C}^{i}(\mathrm{M})$. In addition, for any $f \in \mathrm{C}^{1}(\mathrm{M})$ and compact set $\mathrm{K} \subset \mathrm{M}$, there exists $C \geqslant 0$ such that for all $t \in[0, T]$,

$$
\sup _{x \in \mathrm{K}}\left\{\left|P_{t} f\right|(x)+\left\|\nabla\left(P_{t} f\right)(x)\right\|\right\} \leqslant C .
$$

Proof. We only show the result for $f \in \mathrm{C}^{1}(\mathrm{M})$, the proof for $f \in \mathrm{C}(\mathrm{M})$ is similar and left to the reader. For all $x \in \mathrm{M}$ denote by $\left(X_{t}^{x}\right)_{t \geqslant 0}$ a PDMP starting from $x$ associated with the characteristics $(\varphi, \lambda, Q)$ and defined by Construction 1. Let $\left(S_{n}^{x}\right)_{n \in \mathbb{N}}$ be the jump times of $\left(X_{t}^{x}\right)_{t \geqslant 0}$ for all $x \in \mathrm{M}$ and $\left(\mathcal{F}_{t}\right)_{t \geqslant 0}$ the associated filtration. Let $f \in \mathrm{C}^{1}(\mathrm{M})$, $T \geqslant 0$ and a compact set $\mathrm{K} \subset \mathrm{M}$. For $T=0$, the result is straightforward so we consider $T>0$. Let $\tilde{\mathrm{K}}$ satisfying for all $n \in \mathbb{N},\left(t_{i}\right)_{i \in \llbracket 1, n+1 \rrbracket} \in \mathbb{R}_{+}^{n}, \sum_{i=1}^{n+1} t_{i} \leqslant T$, (i)-(ii) in Definition 16.

Since for all $x \in \mathrm{K}, \mathbb{P}\left(X_{t}^{x} \in \tilde{\mathrm{K}}\right.$, for all $\left.t \in[0, T]\right)=1$, for all $t \in[0, T]$ and $x \in \mathrm{M}$,

$$
\left|P_{t} f(x)\right|=\left|\mathbb{E}\left[f\left(X_{t}\right)\right]\right| \leqslant \sup _{y \in \tilde{\mathrm{K}}}|f|(y) .
$$

Furthermore, $\left(P_{t}\right)_{t \geqslant 0}$ is assumed to be non explosive. Therefore $\sup _{n \in \mathbb{N}} S_{n}^{x}=+\infty$ and we can consider the following decomposition for all $t \in[0, T]$ and $x \in \mathrm{K}$

$$
P_{t} f(x)=\sum_{n=0}^{+\infty} \mathbb{E}\left[\mathbb{1}_{\left[S_{n}^{x}, S_{n+1}^{x}\right)}(t) f\left(X_{t}^{x}\right)\right] .
$$

We show that for all $n \in \mathbb{N}$,

$$
F_{n, t}: x \mapsto \mathbb{E}\left[\mathbb{1}_{\left[S_{n}^{x}, S_{n+1}^{x}\right)}(t) f\left(X_{t}^{x}\right)\right],
$$

is continuously differentiable and in addition there exists $C \geqslant 0$ such that for all $n \in \mathbb{N}$, $t \in[0, T]$

$$
\sup _{x \in \mathrm{K}}\left\|\nabla F_{n, t}(x)\right\| \leqslant C^{n} / n ! .
$$

Assume for the moment that this result holds for any compact set $\mathrm{K} \subset \mathrm{M}$. Then, we have for all $t \in[0, T]$ and compact $\mathrm{K} \subset \mathrm{M}$,

$$
\lim _{N \rightarrow+\infty} \sum_{n=N}^{\infty} \sup _{x \in \mathrm{K}}\left\|\nabla F_{n, t}(x)\right\|=0 .
$$

By (41), it implies that $x \mapsto P_{T} f(x)$ is continuously differentiable on M. In addition, for all compact set $\mathrm{K} \subset \mathrm{M}$, there exists $C \geqslant 0$ such that for all $t \in[0, T],\left\|\nabla P_{t} f(x)\right\|_{\mathrm{K}} \leqslant C$. This result and (40) imply (39). 
We now turn in showing that for all $n \in \mathbb{N}, F_{n}$ is continuously differentiable and (42) holds. We first show this result for $n=0$. In a second time, we make an induction on $n \in \mathbb{N}^{*}$.

For all $x \in \mathrm{K}$ and $t \in[0, T]$, we have

$$
F_{0, t}(x)=f\left(\phi_{t}(x)\right) \exp \left(-\int_{0}^{t} \lambda\left(\phi_{s}(x)\right) \mathrm{d} s\right) .
$$

Therefore, for all $x \in \mathrm{K}$ and $t \in[0, T]$, we obtain by $\mathbf{A} 3$-(ii)-(iii)

$$
\begin{aligned}
\nabla F_{0, t}(x)=\{ & \left.\nabla f\left(\varphi_{t}(x)\right)\right\} \nabla\left(\varphi_{t}\right)(x) \exp \left(-\int_{0}^{t} \lambda\left(\varphi_{s}(x)\right) \mathrm{d} s\right) \\
& +f\left(\varphi_{t}(x)\right)\left[\int_{0}^{t}\left\{\nabla \lambda\left(\varphi_{s}(x)\right) \cdot \nabla\left(\varphi_{s}\right)(x)\right\} \mathrm{d} s\right] \exp \left(-\int_{0}^{t} \lambda\left(\varphi_{s}(x)\right) \mathrm{d} s\right) .
\end{aligned}
$$

Since for all $x \in \mathrm{K}$ and $t \in[0, T], \varphi_{t}(x) \in \tilde{\mathrm{K}}, f \in \mathrm{C}^{1}(\mathrm{M})$ and using $\mathbf{A} 3$-(ii)-(iii), we get there exists $C_{0} \geqslant 0$ such that for all $t \in[0, T]$,

$$
\sup _{x \in \mathrm{K}}\left[\left|F_{0}(x)\right|+\left\|\nabla F_{0}(x)\right\|\right] \leqslant C_{0} .
$$

We now show the result for $n \in \mathbb{N}^{*}$. We first give an explicit expression of $F_{n}$ for all $n \in \mathbb{N}^{*}$. Indeed, we have conditioning successively on $\mathcal{F}_{S_{n+1}^{x}}, \cdots, \mathcal{F}_{S_{1}^{x}}$, for all $x \in \mathrm{K}$ and $t \in[0, T]$

$$
\begin{gathered}
F_{n, t}(x)=\int_{0}^{t} \mathrm{~d} t_{1} \exp \left(-\int_{0}^{t_{1}} \lambda\left\{\varphi_{s_{1}}(x)\right\} \mathrm{d} s_{1}\right) \int_{\mathrm{M}} \mathrm{K}\left(\varphi_{t_{1}}(x), \mathrm{d} x_{1}\right) \\
\int_{0}^{t-t_{1}} \mathrm{~d} t_{2} \exp \left(-\int_{0}^{t_{2}} \lambda\left\{\varphi_{s_{2}}\left(x_{1}\right)\right\} \mathrm{d} s_{2}\right) \int_{\mathrm{M}} \mathrm{K}\left(\varphi_{t_{2}}\left(x_{1}\right), \mathrm{d} x_{2}\right) \\
\begin{array}{r}
\int_{0}^{t-\sum_{i=1}^{n-1} t_{i}} \mathrm{~d} t_{n} \exp \left(-\int_{0}^{t_{n}} \lambda\left\{\varphi_{s_{n}}\left(x_{n-1}\right)\right\} \mathrm{d} s_{n}\right) \int_{\mathrm{M}} \mathrm{K}\left(\varphi_{t_{n}}\left(x_{n-1}\right), \mathrm{d} x_{n}\right) f\left(\varphi_{t-\sum_{i=1}^{n} t_{i}}\left(x_{n}\right)\right) \\
\exp \left(-\int_{0}^{t-\sum_{i=1}^{n} t_{i}} \lambda\left\{\varphi_{s_{n+1}}\left(x_{n}\right)\right\} \mathrm{d} s_{n+1}\right),
\end{array}
\end{gathered}
$$

where $\mathrm{K}$ is the kernel defined on $(\mathrm{M}, \mathcal{B}(\mathrm{M}))$ for all $x \in \mathrm{M}$ and $\mathrm{A} \in \mathcal{B}(\mathrm{M})$ by

$$
\mathrm{K}(x, \mathrm{~A})=\lambda(x) Q(x, \mathrm{~A}) .
$$

We introduce a sequence of operator $\left(Q^{(n)}\right)_{n \in \mathbb{N}^{*}}$, defined for all $g: \mathbb{R}_{+} \times \mathrm{M} \rightarrow \mathbb{R}$, bounded on all compact of $[0, T] \times \mathrm{M}$ and measurable, $t \in[0, T]$ and $x \in \mathrm{M}$ by

$$
\begin{gathered}
Q^{(n)} g(t, x)=\int_{0}^{t} \mathrm{~d} t_{1} \exp \left(-\int_{0}^{t_{1}} \lambda\left\{\varphi_{s_{1}}(x)\right\} \mathrm{d} s_{1}\right) \int_{\mathrm{M}} \mathrm{K}\left(\varphi_{t_{1}}(x), \mathrm{d} x_{1}\right) \\
\cdots \int_{0}^{t-\sum_{i=1}^{n-1} t_{i}} \mathrm{~d} t_{n} \exp \left(-\int_{0}^{t_{n}} \lambda\left\{\varphi_{s_{n}}\left(x_{n-1}\right)\right\} \mathrm{d} s_{n}\right) \int_{\mathrm{M}} \mathrm{K}\left(\varphi_{t_{n}}\left(x_{n-1}\right), \mathrm{d} x_{n}\right) g\left(t-\sum_{i=1}^{n} t_{i}, x_{n}\right) .
\end{gathered}
$$

Taking for $g$ the function $g_{F}:(s, y) \mapsto f\left(\varphi_{s}(y)\right) \exp \left(-\int_{0}^{s} \mathrm{~d} u \lambda\left(\varphi_{u}(y)\right)\right)$, we have

$$
F_{n, t}=Q^{(n)} g_{F}(t, \cdot) \text {. }
$$


Since $f \in \mathrm{C}^{1}(\mathbf{M})$ and by $\mathbf{A} 3$-(iii), $g_{F}$ is measurable, for all $s \in[0, T], y \mapsto g_{F}(s, y)$ is continuously differentiable on $\mathrm{M}$ and satisfies for all $T^{\prime} \in[0, T], \mathrm{K}^{\prime} \subset \mathrm{M}$ compact, $\sup _{s \in\left[0, T^{\prime}\right], y \in \mathrm{K}^{\prime}}\left\{\left|g_{F}(s, y)\right|+\left\|\nabla_{x} g_{F}(s, y)\right\|\right\}<+\infty$. Denote by $\mathrm{G}([0, \mathrm{~T}] \times \mathrm{M})$ the set of measurable functions $g: \mathbb{R}_{+} \times \mathrm{M} \rightarrow \mathbb{R}_{+}$satisfying for all $s \in[0, T], y \mapsto$ $g(s, y)$ is continuously differentiable on $\mathrm{M}$ and for all $T^{\prime} \in[0, T], \mathrm{K}^{\prime} \subset \mathrm{M}$ compact, $\sup _{s \in\left[0, T^{\prime}\right], y \in \mathrm{K}^{\prime}}|g(s, y)|+\left\|\nabla_{x} g(s, y)\right\|<+\infty$. Then, if we show for any $g \in \mathrm{G}([0, \mathrm{~T}] \times \mathrm{M})$ that (1) for all $t \in[0, T]$ and $x \in \mathrm{K}, y \mapsto Q^{(n)} g(t, y)$ is differentiable at $x$; (2) there exists $C \geqslant 0$ such that for all $n \in \mathbb{N}^{*}, x \in \mathrm{K}$ and $t \in[0, T]$,

$$
\left|Q^{(n)} g(t, x)\right|+\left\|\nabla_{x} Q^{(n)} g(t, x)\right\| \leqslant C^{n} /(n !),
$$

(44) and (43) show that (42) holds and the proof is completed.

Note the following relation between $Q^{(n-1)}$ and $Q^{(1)}$ which will be essential to the proof: for all $g: \mathbb{R}_{+} \times \mathrm{M} \rightarrow \mathbb{R}$, bounded on all compact of $\mathbb{R}_{+} \times \mathrm{M}$ and measurable, $t \in[0, T]$ and $x \in \mathrm{M}$

$$
\begin{array}{r}
Q^{(n)} g(t, x)=\int_{0}^{t} \mathrm{~d} t_{1} \exp \left(-\int_{0}^{t_{1}} \lambda\left\{\varphi_{s_{1}}(x)\right\} \mathrm{d} s_{1}\right) \int_{\mathrm{M}} \mathrm{K}\left(\varphi_{t_{1}}(x), \mathrm{d} x_{1}\right) Q^{n-1} g\left(t-t_{1}, x_{1}\right) \\
=Q^{(1)}\left[Q^{(n-1)} g\right](t, x) .
\end{array}
$$

First, we make an induction on $i \in \llbracket 1, n \rrbracket$ to show that for all $i \in \llbracket 1, n \rrbracket$ and $g \in \mathrm{G}$, that $(s, x) \mapsto Q^{(i)} g(s, x) \in \mathrm{G}([0, \mathrm{~T}] \times \mathrm{M})([0, T] \times \mathrm{M})$, which will show that for all $t \in[0, T]$, $y \mapsto Q^{n} g(t, y)$ is continuously differentiable. For $i=1$, note that for all $s \in[0, T]$ and $y \in \mathrm{M}$,

$$
Q^{(1)} g(s, y)=\int_{0}^{s} \mathrm{~d} t_{1} \exp \left(-\int_{0}^{t_{1}} \lambda\left\{\varphi_{s_{1}}(y)\right\} \mathrm{d} s_{1}\right) \int_{M} \mathrm{~K}\left(\varphi_{t_{1}}(y), \mathrm{d} y_{1}\right) g\left(s-t_{1}, y_{1}\right) .
$$

Let $T^{\prime} \in[0, T], \mathrm{K}^{\prime} \subset \mathrm{M}$ be compact and $\tilde{\mathrm{K}}^{\prime}$ given by Definition 16 associated with $\mathrm{K}^{\prime}$ and $T^{\prime}$. Then for all $s \in\left[0, T^{\prime}\right], t_{1} \in[0, s], \varphi_{t_{1}}(y) \in \tilde{\mathrm{K}}^{\prime}$ for all $y \in \mathrm{K}^{\prime}$. Therefore we have by assumption on $g$, A 3 -(ii)-(iii), $\mathrm{K} g\left(s-t_{1}, \cdot\right)\left(\varphi_{t_{1}}(\cdot)\right): y \mapsto \int_{\mathrm{M}} \mathrm{K}\left(\varphi_{t_{1}}(y), \mathrm{d} y_{1}\right) g\left(s-t_{1}, y_{1}\right)$ for all $s \in\left[0, T^{\prime}\right], t_{1} \in[0, s]$, is differentiable and there exists $C \geqslant 0$ such that for all $s \in\left[0, T^{\prime}\right], t_{1} \in[0, s]$

$$
\sup _{y \in \mathrm{K}^{\prime}}\left\{\left|\mathrm{K} g\left(s-t_{1}, \cdot\right)\left(\varphi_{t_{1}}(y)\right)\right|+\left\|\nabla_{x} \mathrm{~K} g\left(s-t_{1}, \cdot\right)\left(\varphi_{t_{1}}(y)\right)\right\|\right\}<C
$$

By (47), we get then the result for $i=1$. The result for $i \in \llbracket 2, n \rrbracket$ is then a straightforward consequence of (46) and the case $i=1$.

We now show that for all $g \in \mathrm{G}([0, \mathrm{~T}] \times \mathrm{M})$ that there exists $C \geqslant 0$ such that for all $x \in \mathrm{K}$ and $t \in[0, T]$, (45) holds. By an induction on $N \in \llbracket 1, n \rrbracket$, we show that for all $t \in[0, T],\left(t_{i}\right)_{i \in \llbracket 1, n-N \rrbracket} \in \mathbb{R}_{+}^{n-N}, \sum_{j=1}^{n-N} t_{j} \leqslant t$, there exists $\left(\mathrm{K}_{i}\right)_{i \in \llbracket 0, n-N \rrbracket}$ satisfying (i)-(ii) in Definition 16 with respect to $\mathrm{K}, T, \tilde{\mathrm{K}},\left(t_{i}\right)_{i \in \llbracket 1, n-N \rrbracket}$ and the following bound holds

$$
\sup _{x_{n-N} \in \mathrm{K}_{n-N}}\left|Q^{N} g\left(t-\sum_{j=1}^{n-N} t_{j}, x_{n-N}\right)\right|+\left\|\nabla Q^{N} g\left(t-\sum_{j=1}^{n-N} t_{j}, x_{n-N}\right)\right\|
$$




$$
\leqslant C_{1}^{N}\left\{\sup _{s \in[0, T], y \in \tilde{\mathrm{K}}}|g(s, y)|+\sup _{s \in[0, T], y \in \tilde{\mathrm{K}}}\left\|\nabla_{x} g(s, y)\right\|\right\}\left(t-\sum_{j=1}^{n-N} t_{j}\right)^{N} /(N !)
$$

where

$$
C_{1}=\|\lambda\|_{\infty, \tilde{\mathrm{K}}}+C_{2}, \quad C_{2}=\sup _{s \in[0, T]}\left\|\nabla_{x} \varphi_{s}(x)\right\|_{\infty, \tilde{\mathrm{K}}}\left(T\|\nabla \lambda\|_{\infty, \tilde{\mathrm{K}}}+\|\Psi\|_{\infty, \tilde{\mathrm{K}}}\right) .
$$

Then, the result for $N=n$ will conclude the proof.

For $N=1$, let $t \in[0, T],\left(t_{i}\right)_{i \in \llbracket 1, n-1 \rrbracket} \in \mathbb{R}_{+}^{n-1}, \sum_{j=1}^{n-1} t_{j} \leqslant t$. Note that for all $y \in \mathrm{M}$, setting $u_{n-1}=\sum_{i=1}^{n-1} t_{i}$,

$Q^{(1)} g\left(t-u_{n-1}, y\right)=\int_{0}^{t-u_{n-1}} \mathrm{~d} t_{n} \mathrm{e}^{-\int_{0}^{t_{n}} \lambda\left\{\varphi_{s_{n}}(y)\right\} \mathrm{d} s_{n}} \int_{\mathrm{M}} \mathrm{K}\left(\varphi_{t_{n}}(y), \mathrm{d} y_{n}\right) g\left(t-u_{n-1}-t_{n}, y_{n}\right)$.

For all $t_{n} \in \mathbb{R}_{+}$, such that $\sum_{i=1}^{n} t_{i}<t$, by $\mathbf{A} 3$-(i), there exists $\left(\mathrm{K}_{i}\right)_{i \in \llbracket 0, n \rrbracket}$ satisfying (i)-(ii) in Definition 16. In particular, $\left(\mathrm{K}_{i}\right)_{i \in \llbracket 0, n-1 \rrbracket}$ only depends on $\left(t_{i}\right)_{i \in \llbracket 1, n-1 \rrbracket}$. Then, using A3, we get for all $x_{n-1} \in \mathrm{K}_{n-1}$,

$$
\begin{aligned}
& \left|Q^{(1)} g\left(t-u_{n-1}, x_{n-1}\right)\right| \\
& \leqslant\|\lambda\|_{\infty, \tilde{\mathrm{K}}} \int_{0}^{t-u_{n-1}} \mathrm{~d} t_{n} \sup \left\{|g|\left(t-u_{n-1}-t_{n}, y_{n}\right): y_{n} \in \operatorname{supp}\left\{Q\left(\varphi_{t_{n}}\left(x_{n-1}\right), \mathrm{d} y_{n}\right)\right\}\right\} \\
& \quad \leqslant\|\lambda\|_{\infty, \tilde{\mathrm{K}}} \int_{0}^{t-u_{n-1}} \mathrm{~d} t_{n} \sup \left\{|g|\left(t-u_{n-1}-t_{n}, y_{n}\right): y_{n} \in \mathrm{K}_{n}\right\},
\end{aligned}
$$

and

$$
\begin{aligned}
& \left\|\nabla_{x} Q^{(1)} g\left(t-u_{n-1}, x_{n-1}\right)\right\| \\
& \leqslant C_{2} \int_{0}^{t-u_{n-1}} \mathrm{~d} t_{n} \sup \left\{|g|\left(t-u_{n-1}-t_{n}, y_{n}\right): y_{n} \in \operatorname{supp}\left\{Q\left(\varphi_{t_{n}}\left(x_{n-1}\right), \mathrm{d} y_{n}\right)\right\}\right\} \\
& +C_{2} \int_{0}^{t-u_{n-1}} \mathrm{~d} t_{n} \sup \left\{\left\|\nabla_{x} g\left(t-u_{n-1}-t_{n}, y_{n}\right)\right\|: y_{n} \in \operatorname{supp}\left\{Q\left(\varphi_{t_{n}}\left(x_{n-1}\right), \mathrm{d} y_{n}\right)\right\}\right\} \\
& \leqslant C_{2} \int_{0}^{t-u_{n-1}} \mathrm{~d} t_{n} \sup \left\{|g|\left(t-u_{n-1}-t_{n}, y_{n}^{1}\right)+\left\|\nabla_{x} g\left(t-u_{n-1}-t_{n}, y_{n}^{2}\right)\right\|: y_{n}^{1}, y_{n}^{2} \in \mathrm{K}_{n}\right\},
\end{aligned}
$$

where $C_{2}$ is given by (49). Combining these two results and using that $\mathrm{K}_{n} \subset \tilde{\mathrm{K}}$ for all $t_{n}, \sum_{i=1}^{n} t_{i}<t$ give (48) for $N=1$.

Now assume that the result holds for $N \in \llbracket 1, n-1 \rrbracket$ and let $\left(t_{i}\right)_{i \in \llbracket 1, n-N-1 \rrbracket} \in \mathbb{R}_{+}^{n-N-1}$. By induction hypothesis, for all $t_{n-N} \in \mathbb{R}_{+}$, such that $\sum_{i=1}^{n-N} t_{i}<t$, there exists $\left(\mathrm{K}_{i}\right)_{i \in \llbracket 0, n-N \rrbracket}$ satisfying (i)-(ii) in Definition 16 with respect to $\mathrm{K}, T, \tilde{\mathrm{K}},\left(t_{i}\right)_{i \in \llbracket 1, n-N \rrbracket}$. Then, using $\mathbf{A} 3$ and (46), we get for all $x_{n-N-1} \in \mathrm{K}_{n-N-1}$, setting $u_{n-N-1}=\sum_{i=1}^{n-N-1} t_{i}$ and $\mathrm{A}_{n-N}=\operatorname{supp}\left\{Q\left(\varphi_{t_{n-N}}\left(x_{n-N-1}\right), \mathrm{d} y_{n-N}\right)\right\}$

$$
\begin{aligned}
& \left|Q^{(N)} g\left(t-u_{n-N-1}, x_{n-N-1}\right)\right| \\
& \leqslant\|\lambda\|_{\infty, \tilde{\mathrm{K}}} \int_{0}^{t-u_{n-N-1}} \mathrm{~d} t_{n-N} \sup \left\{\left|Q^{(N-1)} g\right|\left(t-u_{n-N-1}-t_{n-N}, y_{n-N}\right): y_{n-N} \in \mathrm{A}_{n-N}\right\}
\end{aligned}
$$


$\leqslant\|\lambda\|_{\infty, \tilde{\mathrm{K}}} \int_{0}^{t-u_{n-N-1}} \mathrm{~d} t_{n-N} \sup \left\{\left|Q^{(N-1)} g\right|\left(t-u_{n-N-1}-t_{n-N}, y_{n-N}\right): y_{n-N} \in \mathrm{K}_{n-N}\right\}$, and

$$
\begin{aligned}
& \left\|\nabla_{x} Q^{(N)} g\left(t-u_{n-N-1}, x_{n-N-1}\right)\right\| \\
& \leqslant C_{2} \int_{0}^{t-u_{n-N-1}} \mathrm{~d} t_{n-N} \sup \left\{\left|Q^{(N-1)} g\right|\left(t-u_{n-N-1}-t_{n-N}, y_{n-N}\right): y_{n-N} \in \mathrm{A}_{n-N}\right\} \\
& +C_{2} \int_{0}^{t-u_{n-N-1}} \mathrm{~d} t_{n-N} \sup \left\{\left\|\nabla_{x} Q^{(N-1)} g\left(t-u_{n-N-1}-t_{n-N}, y_{n-N}\right)\right\|: y_{n-N} \in \mathrm{A}_{n-N}\right\} \\
& \leqslant C_{2} \int_{0}^{t-u_{n-N-1}} \mathrm{~d} t_{n-N} \sup \left\{\left|Q^{(N-1)} g\right|\left(t-u_{n-N-1}-t_{n-N}, y_{n-N}\right): y_{n-N} \in \mathrm{K}_{n-N}\right\} \\
& +C_{2} \int_{0}^{t-u_{n-N-1}} \mathrm{~d} t_{n-N} \sup \left\{\left\|\nabla_{x} Q^{(N-1)} g\left(t-u_{n-N-1}-t_{n-N}, y_{n-N}\right)\right\|: y_{n-N} \in \mathrm{K}_{n-N}\right\},
\end{aligned}
$$

where $C_{2}$ is given by (49). Combining these two results using $\mathrm{K}_{n-N} \subset \tilde{\mathrm{K}}$ for all $t_{n-N}$, $\sum_{i=1}^{n-N} t_{i}<t$ and the induction hypothesis conclude the proof of (48).

Remark 18. Theorem 17 can be generalized under the condition that for some $k \in \mathbb{N}^{*}$, the characteristics $(\varphi, \lambda, Q)$ satisfy

(i) the flow $\varphi$ and the Markov kernel $Q$ are compactly compatible;

(ii) $\lambda \in \mathrm{C}^{k}(\mathrm{M})$ and for all $f \in \mathrm{C}^{k}(\mathrm{M}), \lambda Q f \in \mathrm{C}^{k}(\mathrm{M})$ and there exists a locally bounded function $\Psi: \mathrm{M} \rightarrow \mathbb{R}_{+}$such that for all $x \in \mathrm{K}, i \in \llbracket 1, k \rrbracket$,

$$
\left\|\mathrm{D}^{i}(\lambda Q f)(x)\right\| \leqslant\|\Psi\|_{\infty, \mathrm{K}} \sup \left\{\left\|\mathrm{D}^{i} f(y)\right\|: y \in \operatorname{supp}\{Q(x, \cdot)\}, i \in \llbracket 1, k \rrbracket\right\} ;
$$

(iii) we have $(t, x) \mapsto \varphi_{t}(x)$ is $k$-times continuously differentiable on $\mathbb{R}_{+} \times \mathrm{M}$ and for all compact $\mathrm{K} \subset \mathrm{M}$ and $t \geqslant 0$,

$$
\sup \left\{\left\|\mathrm{D}^{i} \varphi_{s}(x)\right\|: s \in[0, t], x \in \mathrm{K}, i \in \llbracket 1, k \rrbracket\right\}<+\infty .
$$

Then for all function $f \in \mathrm{C}^{k}(\mathrm{M})$, and $T \in \mathbb{R}_{+}, P_{T} f \in \mathrm{C}^{k}(\mathrm{M})$. In addition, for all compact set $\mathrm{K} \subset \mathrm{M}$, and $T \in \mathbb{R}_{+}$, there exists $C \geqslant 0$ such that for all $t \in[0, T]$, $\sup _{x \in \mathrm{K}}\left|P_{t} f\right|(x)+\sup _{x \in \mathrm{K}, i \in \llbracket 1, k \rrbracket}\left\|\mathrm{D}^{i} P_{t} f(x)\right\| \leqslant C$.

We say that a Markov semigroup $\left(P_{t}\right)_{t \geqslant 0}$ is Feller if $\mathrm{C}_{0}(\mathrm{M}) \subset \mathrm{B}_{0}(\mathrm{M})$, where $\mathrm{B}_{0}(\mathrm{M})$ is defined by (30), and for any $f \in \mathrm{C}_{0}(\mathrm{M})$, and $t \geqslant 0, P_{t} f \in \mathrm{C}_{0}(\mathrm{M})$, where $\mathrm{C}_{0}(\mathrm{M})$ is equipped with the uniform norm $f \mapsto\|f\|_{\infty}$ We refer to Appendix A for the definition of a core of a closed operator.

Corollary 19. Let $\left(P_{t}\right)_{t \geqslant 0}$ be a non explosive PDMP semigroup on $\mathrm{M}$ with corresponding characteristics $(\varphi, \lambda, Q)$ satisfying $\boldsymbol{A} 2-\boldsymbol{A}$ 3. Then, $\left(P_{t}\right)_{t \geqslant 0}$ is Feller and $\mathrm{C}_{c}^{1}(\mathrm{M})$ is a core for the strong generator of $\left(P_{t}\right)_{t \geqslant 0}$ seen as a semigroup on $\mathrm{C}_{0}(\mathrm{M})$.

Proof. By Proposition 15-(a) and Theorem 17, $\left(P_{t}\right)_{t \geqslant 0}$ is Feller. The second statement is a consequence of Theorem 17 and [13, Proposition 3.3, Chapter 1].

Example - Bouncy Particle Sampler. For the BPS process, $\lambda$ is not in $C^{1}(M)$ so that we cannot apply the previous theory. One aim of the following section will then be to introduce a framework where we may overpass this limitation. 


\section{Core and invariant Distributions of PDMPs}

Building upon the two previous sections, the main purpose of this section is to provide a practical conditions on characteristics $(\varphi, \lambda, Q)$ such that if a probability measure $\mu$ on $(\mathrm{M}, \mathcal{B}(\mathrm{M}))$ satisfies for all $f \in \mathrm{C}_{c}^{1}(\mathrm{M}), \int_{\mathrm{M}} \mathcal{A} f(x) \mathrm{d} \mu(x)=0$, where $\mathcal{A}$ is the extended generator of the semigroup $\left(P_{t}\right)_{t \geqslant 0}$ associated with $(\varphi, \lambda, Q)$, then $\mu$ is invariant for $\left(P_{t}\right)_{t \geqslant 0}$. Since the extended generator of $\left(P_{t}\right)_{t \geqslant 0}$ is an extension of the strong generator $\overline{\mathcal{A}}$, it is enough to show that $\mathrm{C}_{c}^{1}(\mathrm{M})$ is a core for $\overline{\mathcal{A}}$ by [13, Proposition 9.2]. To do so, we introduce the following condition on $\left(P_{t}\right)_{t \geqslant 0}$.

Definition 20. We say that the PDMP semi-group $\left(P_{t}\right)_{t \geqslant 0}$ with characteristics $(\varphi, \lambda, Q)$ is smoothly and compactly approximable if for all $\varepsilon>0$ there exist characteristics $\left(\varphi, \lambda^{\varepsilon}, Q^{\varepsilon}\right)$ satisfying $\boldsymbol{A} 2-\boldsymbol{A} 3$ and

$$
\sup _{\substack{x \in \mathbb{M} \\ \mathrm{A} \in \mathcal{B}(\mathrm{M})}}\left\{\lambda^{\varepsilon}(x) \wedge \lambda(x)\left|Q^{\varepsilon}(x, \mathrm{~A})-Q(x, \mathrm{~A})\right|+\left|\lambda^{\varepsilon}(x)-\lambda(x)\right|\right\} \leqslant \varepsilon .
$$

We may now give the main result of this section.

Theorem 21. Let $\left(P_{t}\right)_{t \geqslant 0}$ be a non explosive PDMP semigroup with characteristics $(\varphi, \lambda, Q)$ which is smoothly and compactly approximable. Then $\left(P_{t}\right)_{t \geqslant 0}$ is a Feller semigroup and $\mathrm{C}_{c}^{1}(\mathrm{M})$ is a core for the strong generator $\overline{\mathcal{A}}$ of $\left(P_{t}\right)_{t \geqslant 0}$ seen as an operator on $\mathrm{C}_{0}(\mathrm{M})$.

Proof. Suppose for the moment that $\left(P_{t}\right)_{t \geqslant 0}$ is Feller. For any $\varepsilon>0$, consider a PDMP semi-group $\left(P_{s}^{\varepsilon}\right)_{s \geqslant 0}$ with characteristics $\left(\varphi, \lambda^{\varepsilon}, Q^{\varepsilon}\right)$ satisfying A 2-A 3 and (50). By Theorem 17 and Corollary 19, for any $n \in \mathbb{N}^{*},\left(P_{s}^{1 / n}\right)_{s \geqslant 0}$ is Feller and for any $t \geqslant 0$, $P_{t}^{1 / n} \mathrm{C}_{c}^{1}(\mathrm{M}) \subset \mathrm{C}_{c}^{1}(\mathrm{M})$. In addition, Theorem 11 implies that for all $x \in \mathrm{M}, n \in \mathbb{N}^{*}$ and $t \geqslant 0,\left\|\delta_{x} P_{t}-\delta_{x} P_{t}^{1 / n}\right\|_{\mathrm{TV}} \leqslant 2\left(1-\mathrm{e}^{-t / n}\right) \leqslant 2 t / n$, and therefore, for any $f \in \mathrm{C}_{0}(\mathrm{M})$,

$$
\left\|P_{t} f-P_{t}^{1 / n} f\right\|_{\infty} \leqslant 2 t\|f\|_{\infty} / n
$$

Then, all the conditions of Proposition 27 in Appendix A are satisfied and we can conclude that $\mathrm{C}_{c}^{1}(\mathrm{M})$ is a core for $\left(P_{t}\right)_{t \geqslant 0}$.

We now prove that $\left(P_{t}\right)_{t \geqslant 0}$ is a Feller semigroup. We first show that for any $f \in \mathrm{C}_{0}(\mathrm{M})$ and $t \geqslant 0, P_{t} f \in \mathrm{C}_{0}(\mathrm{M})$. Indeed, (51) gives that for any $t \geqslant 0$ and $f \in \mathrm{C}_{0}(\mathrm{M}), P_{t} f$ is the uniform limit of $\left(P_{t}^{1 / n} f\right)_{n \in \mathbb{N}^{*}}$, therefore since $\mathrm{C}_{0}(\mathrm{M})$ is closed for the uniform topology, $P_{t} f \in \mathrm{C}_{0}(\mathrm{M})$. Finally, by (51) applied with $n=1$, we have for any $f \in \mathrm{C}_{0}(\mathrm{M})$ and $t>0$, $\left\|P_{t} f-f\right\|_{\infty} \leqslant 2 t+\left\|P_{t}^{1} f-f\right\|_{\infty}$. Since $\left(P_{t}^{1}\right)_{t \geqslant 0}$ is Feller, we get $\lim _{t \downarrow 0}\left\|P_{t} f-f\right\|_{\infty}=0$, which implies that $\mathrm{C}_{0}(\mathrm{M}) \subset \mathrm{B}_{0}(\mathrm{M})$ and completes the proof.

Corollary 22. Let $\left(P_{t}\right)_{t \geqslant 0}$ be a non explosive PDMP semigroup with characteristics $(\varphi, \lambda, Q)$ and $\mu$ be a measure on $(\mathrm{M}, \mathcal{B}(\mathrm{M}))$. Assume $\int_{\mathrm{M}}|\lambda|(x) \mu(\mathrm{d} x)<+\infty$ and that $\left(P_{t}\right)_{t \geqslant 0}$ is smoothly and compactly approximable. In addition, suppose that the generator $\mathcal{A}$ associated with $\left(P_{t}\right)_{t \geqslant 0}$ satisfies for all $f \in \mathrm{C}_{c}^{1}(\mathrm{M})$,

$$
\int_{\mathrm{M}} \mathcal{A} f(x) \mu(\mathrm{d} x)=0 \text {. }
$$

Then $\mu$ is invariant for $\left(P_{t}\right)_{t \geqslant 0}$. 
With this notion of smoothly approximable semigroup, we will be able to consider the BPS process.

\section{Example - Bouncy Particle Sampler.}

Proposition 23. Let $U \in \mathrm{C}^{2}\left(\mathbb{R}^{d}\right), \lambda_{\mathrm{c}}>0$ and $\mu_{\mathrm{v}} \in \mathcal{P}(\mathrm{Y})$. Assume that $\mathrm{Y}$ is bounded. Then the associated BPS on $\mathbb{R}^{d} \times \mathrm{Y}$, given by Example 1, is smoothly and compactly approximable.

Proof. Let $(\varphi, \lambda, Q)$ be the characteristics of the BPS given by Section 4. Let $\varepsilon>0$, $\lambda^{\varepsilon}: \mathrm{M} \rightarrow \mathbb{R}_{+}$and $Q^{\varepsilon}$ be a Markov kernel on $\left(\mathbb{R}^{d} \times \mathrm{Y}, \mathcal{B}\left(\mathbb{R}^{d} \times \mathrm{Y}\right)\right)$ defined for all $(x, y) \in \mathbb{R}^{d} \times \mathrm{Y}$ and $\mathrm{A} \in \mathcal{B}\left(\mathbb{R}^{d}\right)$ by

$$
\begin{gathered}
\lambda^{\varepsilon}(x, y)=\lambda_{1}^{\varepsilon}(x, y)+\lambda_{\mathrm{c}}, \lambda_{1}^{\varepsilon}(x, y)=(\langle y, \nabla U(x)\rangle-\varepsilon)_{+}^{2} /\left(\varepsilon+(\langle y, \nabla U(x)\rangle-\varepsilon)_{+}\right), \\
Q^{\varepsilon}((x, y), \mathrm{A})=\left(1 / \lambda^{\varepsilon}(x, y)\right)\left\{\lambda_{1}^{\varepsilon}(x, y) \boldsymbol{\delta}_{(x, \mathrm{R}(x, y))}(\mathrm{A})+\lambda_{\mathrm{c}}\left(\boldsymbol{\delta}_{x} \otimes \mu_{\mathrm{v}}\right)(\mathrm{A})\right\},
\end{gathered}
$$

where $\mathrm{R}$ is defined by (5) for $g=\nabla U$. Then, similarly to the BPS process, $\left(\varphi, \lambda^{\varepsilon}, Q^{\varepsilon}\right)$ defines a non explosive semi-group $\left(P_{t}^{\varepsilon}\right)_{t \geqslant 0}$ on $\left(\mathbb{R}^{d} \times \mathrm{Y}\right) \times \mathcal{B}\left(\mathbb{R}^{d} \times \mathrm{Y}\right)$. In addition we have

$$
\sup _{(x, y) \in \mathbb{R}^{d} \times \mathbf{Y}}\left|\lambda^{(\varepsilon)}(x, y)-\lambda(x, y)\right| \leqslant 2 \varepsilon .
$$

Therefore, using Remark 12, we get

$$
\begin{gathered}
\sup _{\substack{(x, y) \in \mathbb{R}^{d} \times \mathbf{Y} \\
\mathbf{A} \in \mathcal{B}\left(\mathbb{R}^{d} \times \mathrm{Y}\right)}}\left\{\lambda^{\varepsilon}(x, y) \wedge \lambda(x, y)\left|Q^{\varepsilon}((x, y), \mathrm{A})-Q((x, y), \mathrm{A})\right|+\left|\lambda^{\varepsilon}(x, y)-\lambda(x, y)\right|\right\} \\
\leqslant 2 \sup _{\substack{(x, y) \in \mathbb{R}^{d} \times \mathbf{Y} \\
\mathrm{A} \in \mathcal{B}\left(\mathbb{R}^{d} \times \mathbf{Y}\right)}}\left|\lambda^{\varepsilon}(x, y) Q^{\varepsilon}((x, y), \mathrm{A})-\lambda(x, y) Q((x, y), \mathrm{A})\right| \leqslant 4 \varepsilon
\end{gathered}
$$

which shows (50).

Since $\mathrm{Y}$ is assumed to be bounded, $\mathrm{Y} \subset \overline{\mathrm{B}}\left(0, M_{\mathrm{Y}}\right)$, with $M_{\mathrm{Y}} \in \mathbb{R}_{+}$. Therefore by definition, for all $t \in \mathbb{R}_{+}$and $(x, y) \in \mathbb{R}^{d} \times \mathrm{Y}$, we have $P_{t}^{\varepsilon}\left((x, y), \overline{\mathrm{B}}\left(x, t M_{\mathrm{Y}}\right) \times \mathrm{Y}\right)=1$ and $P_{t}^{\varepsilon}$ satisfies $\mathbf{A} 2$.

Finally, we show that $\mathbf{A} 3$ is satisfied. A3-(iii) trivially holds by definition of $\varphi$.

For all closed ball, $\overline{\mathrm{B}}(0, M) \subset \mathbb{R}^{d}, M \in \mathbb{R}_{+}, Q^{\varepsilon}((x, y), \overline{\mathrm{B}}(0, M) \times \mathrm{Y})=1$ for all $x \in \overline{\mathrm{B}}(0, M)$. For all compact set $\mathrm{K} \subset \mathrm{B}(0, M) \times \mathrm{Y} \subset \mathbb{R}^{d} \times \mathrm{Y}, M \geqslant 0$, and $T \in \mathbb{R}_{+}$, define $\tilde{K}=\mathrm{B}(0, M+T R) \times \mathrm{Y}$, with $R=\sup _{y \in \mathrm{Y}}\|y\|$. Then, for all $n \in \mathbb{N}^{*},\left(t_{i}\right)_{i \in \llbracket 1, n \rrbracket} \in \mathbb{R}_{+}^{n}$, $\sum_{\text {by }}^{n} t_{i} \leqslant T$, conditions (i)-(ii) of Definition 16 are satisfied with $\tilde{K}$, and $\left(\mathrm{K}_{i}\right)_{i \in \llbracket 1, n \rrbracket}$ given

$$
\mathrm{K}_{i}=\overline{\mathrm{B}}\left(0, R_{i}\right) \times \mathrm{Y}, R_{i}=M+R \sum_{j=0}^{i} t_{j}
$$

Thus, $\varphi$ and $Q^{\varepsilon}$ are compactly compatible.

Then note that for all $\varepsilon>0, \lambda^{\varepsilon}$ is continuously differentiable on $\mathrm{M}$ since $t \mapsto(t-$ $\varepsilon)_{+}^{2} /\left(\varepsilon+(t-\varepsilon)_{+}\right)$is on $\mathbb{R}$; and its gradient is given for all $x \in \mathrm{M}$ by

$$
\nabla\left(\lambda^{(\varepsilon)}\right)(x, y)= \begin{cases}\frac{(\langle y, \nabla U(x)\rangle-\varepsilon)(\langle y, \nabla U(x)\rangle+\varepsilon)}{\langle y, \nabla U(x)\rangle^{2}}\left(\nabla^{2} U(x) y, \nabla U(x)\right) & \text { if }\langle y, \nabla U(x)\rangle \geqslant \varepsilon \\ 0 & \text { otherwise }\end{cases}
$$


In addition, for all continuously differentiable function $f: \mathbb{R}^{d} \times \mathrm{Y} \rightarrow \mathbb{R},(x, y) \in \mathbb{R}^{d} \times \mathrm{Y}$, we have $\lambda^{\varepsilon}(x, y) Q^{\varepsilon} f(x, y)=A_{1}(x, y)+A_{2}(x, y)$ where

$$
A_{1}(x, y)=\lambda_{1}^{\varepsilon}(x, y) f(x, \mathrm{R}(x, y)), A_{2}(x, y)=\lambda_{\mathrm{c}} \int_{Y} f(x, \tilde{y}) \mu_{\mathrm{v}}(\mathrm{d} \tilde{y}) .
$$

We show that $A_{1}$ and $A_{2}$ are continuously differentiable and satisfy for $i=1,2$, for all compact set $\mathrm{K} \in \mathbb{R}^{d} \times \mathrm{Y}$, for all $(x, y) \in \mathrm{K}$,

$$
\left\|\nabla A_{i}(x, y)\right\| \leqslant \sup _{(w, z) \in \mathrm{K}}\left\{\Psi_{i}(w, z)\right\} \sup \{|f|(x, \tilde{y})+\|\nabla f(x, \tilde{y})\|: \tilde{y} \in \mathrm{Y}\},
$$

where $\Psi_{i}: \mathbb{R}^{d} \times \mathrm{Y} \rightarrow \mathbb{R}_{+}, i=1,2$, are bounded on compact sets of $\mathbb{R}^{d} \times \mathrm{Y}$. Note that if we show (55), since for all $(x, y) \in \mathbb{R}^{d} \times \mathrm{Y}, \operatorname{supp}\{Q((x, y), \cdot)\}=\{x\} \times \mathrm{Y}$, this result concludes the proof that $\mathbf{A} 3$-(ii) holds.

First, for all $(x, y) \in \mathbb{R}^{d} \times \mathrm{Y}, \mathrm{R}$ is continuously differentiable at $(x, y)$ is $\langle y, \nabla U(x)\rangle \neq 0$. Since $f, \lambda_{1}^{\varepsilon}$ are continuously differentiable and $\lambda_{1}^{\varepsilon}(x, y)=0$ if $\langle y, \nabla U(x)\rangle \leqslant \varepsilon, A_{1}$ is continuously differentiable and satisfies for all $(x, y) \in \mathbb{R}^{d} \times y,\langle y, \nabla U(x)\rangle \geqslant \varepsilon$ $(56)$

$\left\|\nabla A_{1}(x, y)\right\| \leqslant\left\|\nabla \lambda_{1}^{\varepsilon}(x, y)\right\||f|(x, \mathrm{R}(x, y))+\lambda_{1}^{\varepsilon}(x, y)(1+\|\nabla \mathrm{R}(x, y)\|)\|\nabla f(x, \mathrm{R}(x, y))\|$.

Regarding $A_{2}$, we have for all $(x, y) \in \mathbb{R}^{d} \times \mathrm{Y}$, since $\nabla f$ is bounded on all compact sets of $\mathbb{R}^{d} \times \mathrm{Y}$ and the Lebesgue dominated convergence theorem,

$$
\left\|\nabla A_{2}(x, y)\right\| \leqslant \lambda_{\mathrm{c}} \int_{\mathrm{Y}}\left\|\nabla_{x} f(x, \tilde{y})\right\| \mu_{\mathrm{v}}(\tilde{y}) \leqslant \lambda_{\mathrm{c}} \sup _{y \in \mathrm{Y}}\|\nabla f(x, \tilde{y})\|,
$$

where $\nabla_{x}$ is the differential operator with respect to the $x$-variable. Combining this result and (56), we get that (55) holds and therefore A3-(ii) as well.

Corollary 24. Consider the BPS characteristics $\left(\varphi, \lambda_{1}, Q_{1}, \lambda_{2}, Q_{2}\right)$ defined in Example 1 , and let $\left(P_{t}\right)_{t \geqslant 0}$ be the corresponding semigroup. Assume that $\mu_{\mathrm{v}}$ is rotation invariant, i.e. for all $O \in \mathbb{R}^{d \times d}, O^{\mathrm{T}} O=\mathrm{Id}, \mu_{\mathrm{v}}(O \mathrm{~A})=\mu_{\mathrm{v}}(\mathrm{A})$, for all $\mathrm{A} \in \mathcal{B}(\mathrm{Y})$. In addition, suppose that

$$
\int_{\mathbb{R}^{d}}(1+\|\nabla U(x)\|) \mathrm{e}^{-U(x)} \mathrm{d} x<\infty, \quad \int_{\mathrm{Y}}\|y\| \mu_{\mathrm{v}}(\mathrm{d} y)<\infty .
$$

Then $\tilde{\pi}=\pi \otimes \mu_{\mathrm{v}}$ is invariant for $\left(P_{t}\right)_{t \geqslant 0}$, where $\pi$ is the probability measure on $\left(\mathbb{R}^{d}, \mathcal{B}\left(\mathbb{R}^{d}\right)\right)$ with density with respect to the Lebesgue measure proportional to $x \mapsto \mathrm{e}^{-U(x)}$.

Proof. The case where $Y$ is bounded is a direct consequence of Theorem 21.

In the case where $Y$ is not bounded, consider the conditional distribution associated with $\mu_{\mathrm{v}}$ defined for all $\mathrm{A} \in \mathcal{B}(\mathrm{Y})$,

$$
\mu_{\mathrm{v}}^{R}(\mathrm{~A})=\mu_{\mathrm{v}}(\mathrm{B}(0, R) \cap \mathrm{A}) / \mu_{\mathrm{v}}\left(\mathrm{B}\left(x_{0}, R\right)\right),
$$

for $R$ large enough such that $\mu_{\mathrm{v}}(\mathrm{B}(0, R)) \neq 0$. Then, for any $\mathrm{A} \in \mathcal{B}(\mathrm{Y})$, we get $\mid \mu_{\mathrm{v}}(\mathrm{A})-$ $\mu_{\mathrm{v}}^{R}(\mathrm{~A}) \mid \leqslant 2 \mu_{\mathrm{v}}(\mathrm{Y} \backslash \mathrm{B}(0, R))$, which implies that for any $f: \mathbb{R}^{d} \times \mathrm{Y} \rightarrow \mathbb{R}$, bounded and measurable, $t \geqslant 0$, we have for all $R \geqslant 0$ large enough,

$$
\left|\int_{\mathbb{R}^{d} \times \mathbf{Y}} P_{t} f(x, y) \mathrm{d} \tilde{\pi}(x, y)-\int_{\mathbb{R}^{d} \times \mathbf{Y}} f(x, y) \mathrm{d} \tilde{\pi}(x, y)\right|
$$




$$
\leqslant 2\|f\|_{\infty} \mu_{\mathrm{v}}(\mathrm{Y} \backslash \mathrm{B}(0, R))+\left|\int_{\mathbb{R}^{d} \times \mathbf{Y}} P_{t} f(x, y) \mathrm{d} \tilde{\pi}^{R}(x, y)-\int_{\mathbb{R}^{d} \times \mathbf{Y}} P_{t}^{R} f(x, y) \mathrm{d} \tilde{\pi}^{R}(x, y)\right| .
$$

Since $\lim _{R \rightarrow+\infty} \mu_{\mathrm{v}}(\mathrm{Y} \backslash \mathrm{B}(0, R))=0$, it remains to show that the last term in the righthand side goes to 0 as $R \rightarrow+\infty$ and the proof will be finished. Besides, note that this result holds if for $t>0$, we show that for all $(x, y) \in \mathbb{R}^{d} \times \mathrm{Y}$,

$$
\lim _{R \rightarrow+\infty}\left\|\delta_{(x, y)} P_{t}-\delta_{(x, y)} P_{t}^{R}\right\|_{\mathrm{TV}}=0 .
$$

But by Theorem 11 and definition of characteristics of $\left(P_{s}\right)_{s \geqslant 0}$ and $\left(P_{s}^{R}\right)_{s \geqslant 0}$, we get for all $t \geqslant 0,(x, y) \in \mathbb{R}^{d} \times \mathrm{Y}$,

$$
\left\|\delta_{(x, y)} P_{t}-\delta_{(x, y)} P_{t}^{R}\right\|_{\mathrm{TV}} \leqslant 2\left\{1-\exp \left(-2 \lambda_{\mathrm{c}} \mu[\mathrm{Y} \backslash \mathrm{B}(0, R)]\right)\right\},
$$

which shows that (58) holds.

\section{Stability OF INVARIANT MEASURE AND JUmP RATE}

We conclude this work with an asymptotic counterpart of the comparison theorems established in Section 6. For a measurable function $V: \mathrm{M} \rightarrow[1,+\infty)$ and $\nu_{1}, \nu_{2} \in \mathcal{P}(\mathrm{M})$, $\nu_{1}(V), \nu_{2}(V)<+\infty$, define the $V$-norm between $\nu_{1}$ and $\nu_{2}$ by

$$
\left\|\nu_{1}-\nu_{2}\right\|_{V}=\sup \left\{\left|\int_{M} f \mathrm{~d} \nu_{1}-\int_{M} f \mathrm{~d} \nu_{2}\right|,\|f / V\|_{\infty} \leqslant 1\right\} .
$$

We say that a semi-group on $\mathrm{M}$ with invariant probability measure $\mu$ is $V$-uniformly geometrically ergodic with constants $C, \rho>0$ if for all $\nu \in \mathcal{P}(\mathrm{M}), \nu(V)<+\infty$ and $t \geqslant 0$

$$
\left\|\mu-\nu P_{t}\right\|_{V} \leqslant C \mathrm{e}^{-\rho t} \nu(V) .
$$

Theorem 25. Let $\left(P_{t}^{1}\right)_{t \geqslant 0}$ and $\left(P_{t}^{2}\right)_{t \geqslant 0}$ be two non-explosive homogeneous PDMP semigroup with characteristics $\left(\varphi, \lambda_{1}, Q_{1}\right)$ and $\left(\varphi, \lambda_{2}, Q_{2}\right)$ respectively. Let $\mu_{1}, \mu_{2} \in \mathcal{P}(\mathrm{M})$ be invariant for $\left(P_{t}^{1}\right)_{t \geqslant 0}$ and $\left(P_{t}^{2}\right)_{t \geqslant 0}$ respectively. Suppose that $\left(P_{t}^{1}\right)_{t \geqslant 0}$ is $V$-uniformly geometrically ergodic with constants $C, \rho \in \mathbb{R}_{+}^{*}$ for a function $V: \mathrm{M} \rightarrow[1,+\infty)$ such that $\mu_{2}(V)<+\infty$. Assume in addition that

$$
\mathrm{C}_{c}^{1}(\mathrm{M}) \subset \mathrm{B}_{0}^{1}(\mathrm{M})=\left\{f \in \mathrm{B}(\mathrm{M}): \lim _{t \rightarrow 0}\left\|P_{t}^{1} f-f\right\|_{\infty}=0\right\},
$$

and that for all $t \in \mathbb{R}_{+}$and $f \in \mathrm{C}_{c}^{1}(\mathrm{M}), x \mapsto \int_{0}^{t} P_{s}^{1} f(x) \mathrm{d} s \in \mathrm{D}\left(\overline{\mathcal{A}}_{2}\right)$, where $\left(\overline{\mathcal{A}}_{2}, \mathrm{D}\left(\overline{\mathcal{A}}_{2}\right)\right)$ is the strong generator of $\left(P_{t}^{2}\right)_{t \geqslant 0}$. Then

$$
\left\|\mu_{1}-\mu_{2}\right\|_{V} \leqslant C \rho^{-1} \sup \left\{\int_{\mathrm{M}}\left|\lambda_{1} Q_{1} h-\lambda_{2} Q_{2} h+\left(\lambda_{2}-\lambda_{1}\right) h\right| \mathrm{d} \mu_{2}:\|h / V\|_{\infty} \leqslant 1\right\} .
$$

Proof. By density, it is sufficient to bound $\left|\mu_{1}(f)-\mu_{2}(f)\right|$ for all $f \in \mathrm{C}_{c}^{1}(\mathrm{M})$ with $\|f / V\|_{\infty} \leqslant 1$. Let $f \in \mathrm{C}_{c}^{1}(\mathrm{M})$ with $\|f / V\|_{\infty} \leqslant 1$ and, for $t \geqslant 0$, let $g_{t}=\int_{0}^{t} P_{s}^{1}(f-$ $\left.\mu_{1}(f)\right) \mathrm{d} s$. According to [9, Proposition 14.10], for all $t \geqslant 0, g_{t} \in \mathrm{D}\left(\overline{\mathcal{A}}_{1}\right)$ as a sum of a constant function and of $\int_{0}^{t} P_{s} f \mathrm{~d} s$ with $f \in \mathrm{B}_{0}^{1}(\mathrm{M})$, where $\left(\overline{\mathcal{A}}_{1}, \mathrm{D}\left(\overline{\mathcal{A}}_{1}\right)\right)$ is the strong generator of $\left(P_{t}^{1}\right)_{t \geqslant 0}$; moreover it holds

$$
\overline{\mathcal{A}}_{1} g_{t}=\overline{\mathcal{A}}_{1} \int_{0}^{t} P_{s} f \mathrm{~d} s=P_{t} f-f .
$$


Using that $\left(P_{t}^{1}\right)_{t \geqslant 0}$ is $V$-uniformly geometrically ergodic and that $g_{t} \in \mathrm{D}\left(\overline{\mathcal{A}}_{2}\right)$, we obtain for all $t \geqslant 0$ and $x \in \mathrm{M}$ that

$$
\begin{aligned}
\left|\mu_{1}(f)-f(x)-\overline{\mathcal{A}}_{2} g_{t}(x)\right| & \leqslant\left\|\delta_{x} P_{t}-\mu_{1}\right\|_{V}+\left|P_{t} f(x)-f(x)-\overline{\mathcal{A}}_{2} g_{t}(x)\right| \\
& \leqslant C \mathrm{e}^{-\rho t} V(x)+\left|\overline{\mathcal{A}}_{1} g_{t}(x)-\overline{\mathcal{A}}_{2} g_{t}(x)\right| .
\end{aligned}
$$

In addition, by definition of $\left(\overline{\mathcal{A}}_{2}, \mathrm{D}\left(\overline{\mathcal{A}}_{2}\right)\right)$ and since $\mu_{2}$ is invariant for $\left(P_{t}^{2}\right)_{t \geqslant 0}$, then $\mu_{2}\left(\overline{\mathcal{A}}_{2} g_{t}\right)=0$ for all $t \geqslant 0$, so that

$$
\left|\mu_{1}(f)-\mu_{2}(f)\right|=\left|\mu_{2}\left\{\mu_{1}(f)-f-\overline{\mathcal{A}}_{2} g_{t}\right\}\right| \leqslant \mu_{2}\left(\left|\left(\overline{\mathcal{A}}_{1}-\overline{\mathcal{A}}_{2}\right) g_{t}\right|\right)+C \mathrm{e}^{-\rho t} \mu_{2}(V) .
$$

Since $\mathrm{D}\left(\overline{\mathcal{A}}_{i}\right) \subset \mathrm{D}\left(\mathcal{A}_{i}\right), i=1,2$, for all $t \geqslant 0,\left(\overline{\mathcal{A}}_{1}-\overline{\mathcal{A}}_{2}\right) g_{t}=\left(\mathcal{A}_{1}-\mathcal{A}_{2}\right) g_{t}$. Finally, $\left(P_{t}^{1}\right)_{t \geqslant 0}$ being $V$-uniformly geometrically ergodic, then for all $x \in \mathrm{M}, g_{t}(x) \leqslant(C / \rho) V(x)$. The proof is then concluded taking $t \rightarrow+\infty$.

Example - Bouncy Particle Sampler. Let us apply this result in the case of the Bouncy Particle Sampler. Ergodicity of the BPS is studied in [12], to which we will refer for details on this matter in the following. For the sake of simplicity, we will work under restrictive conditions.

Proposition 26. Consider the BPS with characteristics $\left(\varphi, \lambda_{1}, Q_{1}, \lambda_{2}, Q_{2}\right)$ defined in Example 1, with $U \in \mathrm{C}^{2}(\mathrm{M})$ and $\mathrm{Y} \subset \overline{\mathrm{B}}(0,1)$, and $\left(P_{t}\right)_{t \geqslant 0}$ the corresponding semigroup. For $M>0$, let $\left(P_{t}^{M}\right)_{t \geqslant 0}$ be the PDMP semi-group with characteristics $\left(\varphi, \lambda_{1} \wedge\right.$ $\left.M, Q_{1}, \lambda_{2}, Q_{2}\right)$.

Assume that $\mu_{\mathrm{v}}$ is rotation invariant and (57) holds. In addition, assume that there exist $R>0, W \in \mathrm{C}^{2}\left(\mathbb{R}^{d}\right)$ and $F \in \mathrm{C}^{2}(\mathbb{R})$ such that $U(x)=F(W(x))$ for $x \notin \mathrm{B}(0, R)$, $\|\nabla W\|_{\infty}+\left\|\nabla^{2} W\right\|_{\infty}<+\infty, \int_{\mathrm{M}} \exp (-W(x)) \mathrm{d} x<\infty, \lim _{\|x\| \rightarrow+\infty} W(x)=+\infty$ and $\lim _{w \in+\infty} F^{\prime}(w)=+\infty$. Then there exists $C>0$ and $\tilde{M}>0$ such that for all $M>\tilde{M}$, $\left(P_{t}^{M}\right)_{t \geqslant 0}$ admits a unique invariant measure $\tilde{\pi}_{M}$ that satisfies

$$
\left\|\tilde{\pi}-\tilde{\pi}_{M}\right\|_{\mathrm{e}^{W}} \leqslant C \int_{\mathrm{M}}(\|\nabla U(x)\|-M)_{+} \mathrm{e}^{W(x)-U(x)} \mathrm{d} x,
$$

where $\tilde{\pi}=\pi \otimes \mu_{\mathrm{v}}$ where $\pi$ is the probability measure on $\left(\mathbb{R}^{d}, \mathcal{B}\left(\mathbb{R}^{d}\right)\right)$ with density with respect to the Lebesgue measure proportional to $x \mapsto \mathrm{e}^{-U(x)}$.

For example, if $U(x)=\langle x, A x\rangle$ for some definite positive matrix $A$ outside a ball, then these conditions are satisfied by $W(x)=\sqrt{1+U(x)}$ and $F(w)=w^{2}-1$. In this case, Proposition 26 implies that there exist $M_{0}, C, c>0$ such that for all $M>M_{0}$,

$$
\left\|\tilde{\pi}-\tilde{\pi}_{M}\right\|_{\mathrm{e}^{W}} \leqslant C \mathrm{e}^{-c M^{2}} .
$$

Note that for all $M>0,\left(P_{t}^{M}\right)_{t \geqslant 0}$ can be sampled by thinning procedures, see $[17,16]$.

Proof. For all $\varepsilon>0$, in a similar way as in Proposition 23, we can construct a semi-group $\left(P_{t}^{M, \varepsilon}\right)_{t \geqslant 0}$ with characteristics $\left(\varphi, \lambda_{M, \varepsilon}, Q_{1}, \lambda_{2}, Q_{2}\right)$ where $\lambda_{M, \varepsilon}$ is such that $\left(P_{t}^{M, \varepsilon}\right)_{t \geqslant 0}$ satisfies A2-A3 and that

$$
\sup _{(x, y) \in \mathbb{R}^{d} \times Y}\left|\lambda_{1}(x, y) \wedge M-\lambda_{M, \varepsilon}(x, y)\right| \leqslant \varepsilon .
$$

Let us show that we can apply Theorem 25 twice, with each time $P_{t}^{1}=P_{t}^{M, \varepsilon}$ and $P_{t}^{2}$ equal either to $P_{t}^{M}$ or $P_{t}$. Consider the Lyapunov function defined for all $(x, y) \in \mathbb{R}^{d} \times \mathrm{Y}$ 
by $V(x, y)=\exp (W(x)) \phi(\langle y, \nabla W(x)\rangle)$, where $\phi \in \mathrm{C}^{2}(\mathbb{R})$ is an increasing function, with $\phi(r)=1$ for $r \leqslant-2$ and $\phi(r) \leqslant 3$ for $r \geqslant 1$.

We show first that $\left(P_{t}\right)_{t \geqslant 0},\left(P_{t}^{M}\right)_{t \geqslant 0}$ and $\left(P_{t}^{M, \varepsilon}\right)_{t \geqslant 0}$ are $V$-uniformly geometrically ergodic, which will imply that all these semi-groups admit a unique stationary measure for which $V$ is integrable. For $h>0$, let $\mathcal{A}_{h W}$ be the generator of the BPS semi-group with potential $h W$, refreshment rate $\lambda_{\mathrm{c}}>0$ and refreshment law $\mu_{\mathrm{v}}$. Following [12, Section 3.2], there exist $\phi: \mathbb{R} \rightarrow[1,3]$ and $h_{0}>0$ such that there exist $\alpha, C>0$ satisfying

$$
\mathcal{A}_{h_{0} W} V \leqslant-\alpha V+C
$$

Moreover, for all $M>0$, denoting $\mathcal{A}_{M}$ the generator of $\left(P_{t}^{M}\right)_{t \geqslant 0}$,

$$
\left(\mathcal{A}_{M}-\mathcal{A}_{h_{0} W}\right) V(x, y)=\left[\lambda_{1}(x, y) \wedge M-h_{0}\langle y, \nabla W(x)\rangle_{+}\right][\phi(-\langle y, \nabla W(x)\rangle)-\phi(\langle y, \nabla W(x)\rangle)] \mathrm{e}^{W(x)} .
$$

Note that, since $\mathrm{Y} \subset \overline{\mathrm{B}}(0,1), W$ is Lipschitz and $F^{\prime}$ and $W$ going to infinity at infinity, then for $M_{0}=h_{0}\|\nabla W\|_{\infty}$ and some $R_{0}>R$ large enough, for all $M \geqslant M_{0}, x \notin \mathrm{B}\left(0, R_{0}\right)$ and $y \in \mathrm{Y}$,

$$
\begin{aligned}
\lambda_{1}(x, y) \wedge M-h_{0}\langle y, \nabla W(x)\rangle_{+} & \geqslant\left(\left(F^{\prime}(W(x))-h_{0}\right)\langle y, \nabla W(x)\rangle_{+}\right) \wedge\left(M-h_{0}\|\nabla W\|_{\infty}\right) \\
& \geqslant 0 .
\end{aligned}
$$

Besides, $\phi$ being increasing, $\phi(-r)-\phi(r) \leqslant 0$ for all $r \geqslant 0$, so that for all $M \geqslant M_{0}$, $x \notin \mathrm{B}\left(0, R_{0}\right)$ and $y \in \mathrm{Y}$,

$$
\mathcal{A}_{M} V(x, y) \leqslant \mathcal{A}_{h_{0} W} V(x, y) \leqslant-\alpha V(x, y)+C .
$$

Hence, for $M \geqslant M_{0}$ and all $(x, y) \in \mathbb{R}^{d} \times \mathrm{Y}$,

$$
\mathcal{A}_{M} V(x, y) \leqslant-\alpha V(x, y)+C+\sup _{(x, y) \in \mathrm{B}\left(0, R_{0}\right) \times \mathrm{Y}}\left|\mathcal{A}_{M} V(x, y)+\alpha V(x, y)\right| \leqslant-\alpha V(x, y)+C^{\prime},
$$

for some $C^{\prime}$ that does not depend on $M>M_{0}$, since $\lambda_{1}$ is bounded on $\mathrm{B}\left(0, R_{0}\right) \times \mathrm{Y}$. By a similar argument, denoting $\mathcal{A}_{M, \varepsilon}$ the generator of $\left(P_{t}^{M, \varepsilon}\right)_{t \geqslant 0}$,

$$
\mathcal{A}_{M, \varepsilon} V=\mathcal{A}_{M} V+\left(\mathcal{A}_{M, \varepsilon}-\mathcal{A}_{M}\right) V \leqslant-\alpha V+6 \varepsilon \mathrm{e}^{W}+C \leqslant-(\alpha-12 \varepsilon) V+C .
$$

Hence, we have obtained $M_{0}, \varepsilon_{0}, \alpha^{\prime}, C^{\prime}>0$ such that for all $M>M_{0}$, all $\varepsilon \in\left[0, \varepsilon_{0}\right]$ then

$$
\mathcal{A}_{M, \varepsilon} V \leqslant-\alpha^{\prime} V+C^{\prime} \text {. }
$$

Moreover, following [12, Section 3.3], for all compact set $\mathrm{K} \subset \mathbb{R}^{d} \times \mathrm{Y}$, there exist $\eta, t_{0}>0$ such that, for all $M>M_{0}$ and $\varepsilon \in\left[0, \varepsilon_{0}\right]$, for all $(x, y),\left(x^{\prime}, y^{\prime}\right) \in \mathrm{K}$ and all $t \geqslant t_{0}$

$$
\left\|\delta_{x, y} P_{t}^{M, \varepsilon}-\delta_{x^{\prime}, y^{\prime}} P_{t}^{M, \varepsilon}\right\|_{\mathrm{TV}} \leqslant 2(1-\eta) .
$$

Note that, indeed, $\eta$ and $t_{0}$ do not depend on $M$ or $\varepsilon$ since their construction only involves the supremum of $\lambda_{M, \varepsilon}$ over some compact set, which is smaller than $\varepsilon_{0}$ plus the supremum of $\lambda_{1}$ over the same compact (see [12, Section 3.3] for details).

By [19, Theorem 6.1], (60) together with (61) implies that $\left(P_{t}^{M, \varepsilon}\right)_{t \geqslant 0}$ admits a unique invariant measure $\tilde{\pi}_{M, \varepsilon}, \tilde{\pi}_{M, \varepsilon}(V)<+\infty$ and is $V$-uniformly ergodic for all $M>M_{0}$ and $\varepsilon \in\left[0, \varepsilon_{0}\right]$ with some constants that does not depend on $M$ nor $\varepsilon$ (see [12, Section 3] for 
details). More precisely for all $M>M_{0}$ and $\varepsilon \in\left[0, \varepsilon_{0}\right]$, there exists $C \geqslant 0$ and $\rho>0$ such that for all initial distribution $\nu_{0}, \nu_{0}(V)<+\infty$,

$$
\left\|\nu_{0} P_{t}^{M, \varepsilon}-\tilde{\pi}_{M, \varepsilon}\right\|_{\mathrm{TV}} \leqslant C \rho^{t} \nu_{0}(V) .
$$

The case $\varepsilon=0$ in particular implies that $\left(P_{t}^{M}\right)_{t \geqslant 0}$ admits an invariant measure $\tilde{\pi}_{M}$ that satisfies $\tilde{\pi}_{M}(V)<+\infty$. Besides, note that for $R_{1}>R_{0}$ large enough to ensure that $F(W(x)) \geqslant 2 W(x)$ for all $x \notin \mathrm{B}\left(0, R_{1}\right)$,

$$
\int_{\mathrm{M}} \mathrm{e}^{W(x)-U(x)} \mathrm{d} x \leqslant \int_{\mathrm{B}\left(0, R_{1}\right)} \mathrm{e}^{W(x)-U(x)} \mathrm{d} x+\int_{\mathbb{R}^{d} \backslash \mathrm{B}\left(0, R_{1}\right)} \mathrm{e}^{-W(x)} \mathrm{d} x<+\infty,
$$

so that $\tilde{\pi}(V)<+\infty$. Finally, note that $V \leqslant \mathrm{e}^{W} \leqslant 2 V$, so that the associated $V$-norms are equivalent.

In order to apply Theorem 25, it remains to check the regularity conditions. Since $\left(P_{t}^{M, \varepsilon}\right)_{t \geqslant 0}$ satisfies A2-A3, by Theorem 17-Lemma 14, $\int_{0}^{t} P_{s}^{M, \varepsilon} f \mathrm{~d} s \in \mathrm{C}_{c}^{1}\left(\mathbb{R}^{d} \times \mathrm{Y}\right)$ for all $f \in \mathrm{C}_{c}^{1}\left(\mathbb{R}^{d} \times \mathrm{Y}\right), t \geqslant 0, M, \varepsilon>0$. By Proposition 15 , for all $M, \varepsilon>0, \mathrm{C}_{c}^{1}\left(\mathbb{R}^{d} \times \mathrm{Y}\right) \subset$ $\mathrm{D}\left(\overline{\mathcal{A}}_{M, \varepsilon}\right) \cap \mathrm{D}\left(\overline{\mathcal{A}}_{M}\right) \cap \mathrm{D}(\overline{\mathcal{A}})$, where $\overline{\mathcal{A}}$ is the strong generator of $\left(P_{t}\right)_{t \geqslant 0}$. As a consequence, we can apply twice Theorem 25 which implies, combining with (62), that there exists $C_{1} \geqslant 0$ satisfying for any $M>M_{0}$ and $\varepsilon \in\left[0, \varepsilon_{0}\right]$,

$$
\begin{aligned}
\left\|\tilde{\pi}-\tilde{\pi}_{M}\right\|_{\mathrm{e}^{W}} & \leqslant\left\|\tilde{\pi}-\tilde{\pi}_{M, \varepsilon}\right\|_{\mathrm{e}^{W}}+\left\|\tilde{\pi}_{M, \varepsilon}-\tilde{\pi}_{M}\right\|_{\mathrm{e} W} \\
& \leqslant C_{1} \sup \left\{\int_{\mathbb{R}^{d} \times \mathrm{Y}}\left|\lambda_{1}-\lambda_{M, \varepsilon}\right|\left(\left|Q_{1} h\right|+|h|\right) \mathrm{d} \tilde{\pi}:\left\|h \mathrm{e}^{-W}\right\|_{\infty} \leqslant 1\right\} \\
& +C_{1} \sup \left\{\int_{\mathbb{R}^{d} \times \mathrm{Y}}\left|\lambda_{1} \wedge M-\lambda_{M, \varepsilon}\right|\left(\left|Q_{1} h\right|+|h|\right) \mathrm{d} \tilde{\pi}_{M}:\left\|h \mathrm{e}^{-W}\right\|_{\infty} \leqslant 1\right\},
\end{aligned}
$$

Using that $Q_{1} \mathrm{e}^{W}=\mathrm{e}^{W},(59), \tilde{\pi}\left(\mathrm{e}^{W}\right)$ and $\tilde{\pi}_{M}\left(\mathrm{e}^{W}\right)<+\infty$, we obtain that there exists $C_{2} \geqslant 0$ such that

$$
\left\|\tilde{\pi}-\tilde{\pi}_{M}\right\|_{\mathrm{e}^{W}} \leqslant C_{2}\left[\int_{\mathbb{R}^{d} \times \mathrm{Y}}\left\{\lambda_{1}-\lambda_{1} \wedge M\right\} \mathrm{e}^{W} \mathrm{~d} \tilde{\pi}+\varepsilon\right] .
$$

Finally, the proof is concluded taking $\varepsilon \rightarrow 0$ and upon noting that for all $(x, y) \in \mathbb{R}^{d} \times \mathrm{Y}$,

$$
\begin{aligned}
& \lambda_{1}(x, y)-\lambda_{1}(x, y) \wedge M=\left(\langle y, \nabla U(x)\rangle_{+}-M\right) \mathbb{1}_{[M,+\infty)}\left(\langle y, \nabla U(x)\rangle_{+}\right) \\
& \leqslant(\|\nabla U(x)\|-M) \mathbb{1}_{[M,+\infty)}\left(\langle y, \nabla U(x)\rangle_{+}\right) \leqslant(\|\nabla U(x)\|-M)_{+} .
\end{aligned}
$$

\section{ACKNOWLEDGEMENTS}

Alain Durmus acknowledges support from Chaire BayeScale "P. Laffitte". Pierre Monmarché acknowledges support from the French ANR project ANR-12-JS01-0006 PIECE. Arnaud Guillin and Pierre Monmarché acknowledge support from the French ANR-17-CE40-0030 - EFI - Entropy, flows, inequalities. 


\section{REFERENCES}

[1] R. Azaïs, J.-B. Bardet, A. Génadot, N. Krell, and P.-A. Zitt. Piecewise deterministic Markov process - recent results. In Journées MAS 2012, volume 44 of ESAIM Proc., pages 276-290. EDP Sci., Les Ulis, 2014.

[2] M. Benaïm, S. Le Borgne, F. Malrieu, and P.-A. Zitt. Qualitative properties of certain piecewise deterministic Markov processes. Ann. Inst. Henri Poincaré Probab. Stat., 51(3):1040-1075, 2015.

[3] D. P. Bertsekas and S. E. Shreve. Stochastic optimal control, volume 139 of Mathematics in Science and Engineering. Academic Press, Inc. [Harcourt Brace Jovanovich, Publishers], New York-London, 1978. The discrete time case.

[4] J. Bierkens, P. Fearnhead, and G. Roberts. The zig-zag process and super-efficient sampling for bayesian analysis of big data. Ann. Statist., 47(3):1288-1320, 062019.

[5] A. Bouchard-Côté, S. J. Vollmer, and A. Doucet. The bouncy particle sampler: A nonreversible rejection-free markov chain monte carlo method. Journal of the American Statistical Association, 113(522):855-867, 2018.

[6] E. Bouin, J. Dolbeault, S. Mischler, C. Mouhot, and C. Schmeiser. Hypocoercivity without confinement. Pure and Applied Analysis, 2(2):203-232, 2020.

[7] V. Calvez, G. Raoul, and C. Schmeiser. Confinement by biased velocity jumps: aggregation of escherichia coli. Kinet. Relat. Models, 8(4):651-666, 2015.

[8] O. L. V. Costa and F. Dufour. Stability and ergodicity of piecewise deterministic markov processes. SIAM Journal on Control and Optimization, 47:1053-1077, 2008.

[9] M. Davis. Markov Models \& Optimization, volume 49. CRC Press, 1993.

[10] G. Deligiannidis, A. Bouchard-Côté, and A. Doucet. Exponential ergodicity of the bouncy particle sampler. Ann. Statist., 47(3):1268-1287, 062019.

[11] R. Douc, E. Moulines, P. Priouret, and P. Soulier. Markov chains. Springer Series in Operations Research and Financial Engineering. Springer, Cham, 2018.

[12] A. Durmus, A. Guillin, and P. Monmarché. Geometric ergodicity of the bouncy particle sampler. Ann. Appl. Probab., 30(5):2069-2098, 102020.

[13] S. N. Ethier and T. G. Kurtz. Markov processes. Wiley Series in Probability and Mathematical Statistics: Probability and Mathematical Statistics. John Wiley \& Sons Inc., New York, 1986. Characterization and convergence.

[14] M. Jacobsen. Point process theory and applications. Probability and its Applications. Birkhäuser Boston, Inc., Boston, MA, 2006. Marked point and piecewise deterministic processes.

[15] J. Jacod and A. N. Shiryaev. Limit theorems for stochastic processes, volume 288 of Grundlehren der Mathematischen Wissenschaften [Fundamental Principles of Mathematical Sciences]. SpringerVerlag, Berlin, second edition, 2003.

[16] V. Lemaire, M. Thieullen, and N. Thomas. Exact simulation of the jump times of a class of Piecewise Deterministic Markov Processes. J Sci Comput, 75:1776-1807, 2018.

[17] P. A. W. Lewis and G. S. Shedler. Simulation of nonhomogeneous Poisson processes by thinning. Naval Res. Logist. Quart., 26(3):403-413, 1979.

[18] F. Malrieu. Some simple but challenging Markov processes. Ann. Fac. Sci. Toulouse Math. (6), 24(4):857-883, 2015.

[19] S. P. Meyn and R. L. Tweedie. Stability of Markovian processes. III. Foster-Lyapunov criteria for continuous-time processes. Adv. in Appl. Probab., 25(3):518-548, 1993.

[20] M. Michel, A. Durmus, and S. Sénécal. Forward event-chain monte carlo: Fast sampling by randomness control in irreversible markov chains. Journal of Computational and Graphical Statistics, 0(0):1-14, 2020.

[21] P. Monmarché. Piecewise deterministic simulated annealing. ALEA Lat. Am. J. Probab. Math. Stat., 13(1):357-398, 2016.

[22] E. A. J. F. Peters and G. de With. Rejection-free monte carlo sampling for general potentials. Phys. Rev. E 85, 026703, 2012.

[23] P. Vanetti, A. Bouchard-Côté, G. Deligiannidis, and A. Doucet. Piecewise Deterministic Markov Chain Monte Carlo. arXiv preprint arXiv:1707.05296, 2017. 
[24] C. Villani. Optimal transport, volume 338 of Grundlehren der Mathematischen Wissenschaften [Fundamental Principles of Mathematical Sciences]. Springer-Verlag, Berlin, 2009. Old and new.

[25] C. Wu and C. P. Robert. Generalized bouncy particle sampler. arXiv preprint arXiv:1706.04781, 2017.

\section{Appendix A. On Generators of CONTRACTION SEMIGRoups AND THEIR CORE}

In this section, we detail how to establish that a particular subspace is a core for the generator of a contraction semigroup. In all this section, we consider a semigroup $\left(T_{t}\right)_{t \geqslant 0}$ on a Banach space $L$ equipped with the norm $\|\cdot\|_{L}$. We assume the following condition on $\left(T_{t}\right)_{t \geqslant 0}$ :

C1. The semigroup $\left(T_{t}\right)_{t \geqslant 0}$ is a strongly continuous contraction on $\mathbf{L}$ i.e. for any $f \in \mathbf{L}$, $\lim _{s \rightarrow 0}\left\|T_{s} f-f\right\|_{\mathrm{L}}=0$ and for any $t \geqslant 0,\left\|T_{t} f\right\|_{\mathrm{L}} \leqslant\|f\|_{\mathrm{L}}$.

Next, we define the generator $\mathcal{L}$ and its domain of $\left(T_{t}\right)_{t \geqslant 0}$ defined by

$$
\begin{aligned}
\mathrm{D}(\mathcal{L}) & =\left\{f \in \mathrm{L}: \text { there exists } g \in \mathrm{L}, \lim _{t \rightarrow+\infty}\left\|t^{-1}\left(T_{t} f-f\right)-g\right\|_{\mathrm{L}}=0\right\} \\
\mathcal{L} f & =g \in \mathrm{L}, \text { such that } \lim _{t \rightarrow+\infty}\left\|t^{-1}\left(T_{t} f-f\right)-g\right\|_{\mathrm{L}}=0 .
\end{aligned}
$$

Note that by the Hille-Yosida theorem [13, Theorem 2.6], $\mathrm{D}(\mathcal{L})$ is dense in $\mathrm{L}, \operatorname{Ran}(\lambda \operatorname{Id}-\mathcal{L})$ is dense in $\mathrm{L}$ for some $\lambda>0$, where $\operatorname{Ran}(\mathcal{C})$ stands for the range of an operator $\mathcal{C}$ in $\mathrm{L}$, and $\mathcal{L}$ is dissipative, i.e. for any $f \in \mathrm{D}(\mathcal{L})$ and $\lambda>0,\|(\lambda \operatorname{Id}-\mathcal{L}) f\|_{\mathrm{L}} \geqslant \lambda\|f\|_{\mathrm{L}}$.

We are now interested in identifying a core for $\mathcal{L}$. Recall that a core $\mathcal{C}$ of a closed operator $\mathcal{L}$ with domain $\mathrm{D}(\mathcal{L})$ on $\mathrm{L}$ is a subspace of $\mathrm{D}(\mathcal{L})$ such that the closure of the restriction $\left.\mathcal{L}\right|_{C}$ of $\mathcal{L}$ to $\mathrm{C}$ is equal to $\mathcal{L}$. Our main tool is the following result which is in essence a reformulation of [13, Proposition 3.7].

Proposition 27. Assume $\boldsymbol{C} 1$ and that there exists a sequence $\left\{\left(T_{t}^{n}\right)_{t \geqslant 0}: n \in \mathbb{N}^{*}\right\}$ of semigroups satisfying $\boldsymbol{C} 1$ and for any $t \geqslant 0, n \in \mathbb{N}$ and $f \in \mathrm{L}$,

$$
\left\|T_{t} f-T_{t}^{n} f\right\|_{\mathrm{L}} \leqslant \varepsilon_{n} t\|f\|_{\mathrm{L}},
$$

for a sequence $\left(\varepsilon_{n}\right)_{n \in \mathbb{N}^{*}}$ such that $\lim _{n \rightarrow+\infty} \varepsilon_{n}=0$. Suppose in addition that there exists a dense subspace $\mathrm{C}$ of $\mathrm{L}$ such that $\mathrm{C} \subset \mathrm{D}(\mathcal{L})$ and that $T_{t}^{n} f \in \mathrm{C}$ for any $t \geqslant 0, n \in \mathbb{N}^{*}$, $f \in \mathrm{C}$. Then $\mathrm{C}$ is a core for $\left(T_{t}\right)_{t \geqslant 0}$.

Proof. We show that

$$
\mathrm{C} \in \mathrm{Cl}\left(\operatorname{Ran}\left(\left.\{\lambda \operatorname{Id}-\mathcal{L}\}\right|_{\mathrm{C}}\right)\right)
$$

where $\mathrm{Cl}(\mathrm{D})$ is the closure of $\mathrm{D} \subset \mathrm{L}$ in $\mathrm{L}$. Indeed, if this statement holds and since $\mathrm{C}$ is dense, then [13, Proposition 3.1] completes the proof.

First, note that $\mathrm{C} \subset \mathrm{D}\left(\mathcal{L}^{n}\right)$ for any $n \in \mathbb{N}^{*}$, where $\mathcal{L}^{n}$ is the generator associated with $\left(T_{t}^{n}\right)_{t \geqslant 0}$ since $\mathrm{C}$ is a core for $\mathcal{L}^{n}$ by [13, Proposition 3.3]. We now show that for any $n \in \mathbb{N}^{*}$ and $f \in \mathrm{C} \subset \mathrm{D}(\mathcal{L}) \cap \mathrm{D}\left(\mathcal{L}^{n}\right),\left\|\mathcal{L} f-\mathcal{L}^{n} f\right\|_{\mathrm{L}} \leqslant \varepsilon_{n}$. Indeed, we have by (63),

$$
\left\|\mathcal{L} f-\mathcal{L}^{n} f\right\|_{\mathrm{L}} \leqslant \limsup _{s \rightarrow 0} s^{-1}\left\|T_{s} f-T_{s}^{n} f\right\|_{\mathrm{L}} \leqslant \varepsilon_{n} .
$$


We now turn in showing (64). Let $f \in \mathrm{C}$ and for any $n \in \mathbb{N}^{*}$ and $p \in \mathbb{N}^{*}$, define

$$
f_{p}^{n}=p^{-1} \sum_{k=1}^{p^{2}} \mathrm{e}^{-\lambda k / p} T_{k / p}^{n} f .
$$

Note that for any $n, p \in \mathbb{N}^{*}, f_{p}^{n} \in \mathrm{C}$ by assumption on $\left\{\left(T_{t}^{n}\right)_{t \geqslant 0}: n \in \mathbb{N}^{*}\right\}$ and $\left\|f_{p}^{n}\right\|_{\mathrm{L}} \leqslant \lambda^{-1} C\|f\|_{\mathrm{L}}$ for some constant $C \geqslant 0$ independent of $n, p$ and by [13, Proposition 2.1, Proposition 1.5-(b)] for any $n \in \mathbb{N}^{*}$,

$$
\begin{aligned}
\lim _{p \rightarrow+\infty}\left(\lambda \mathrm{Id}-\mathcal{L}^{n}\right) f_{p}^{n} & =\left(\lambda \operatorname{Id}-\mathcal{L}^{n}\right) \int_{0}^{+\infty} \mathrm{e}^{-\lambda s} T_{s}^{n} f \mathrm{~d} s=\int_{0}^{+\infty} \mathrm{e}^{-\lambda s} T_{s}^{n}\left(\lambda \mathrm{Id}-\mathcal{L}^{n}\right) f \mathrm{~d} s \\
& =\left(\lambda \operatorname{Id}-\mathcal{L}^{n}\right)^{-1}\left(\lambda \mathrm{Id}-\mathcal{L}^{n}\right) f=f .
\end{aligned}
$$

Then for any $n \in \mathbb{N}^{*}$, there exists $p_{n} \in \mathbb{N}^{*}$, such that $\left\|f-\left(\lambda \operatorname{Id}-\mathcal{L}^{n}\right) f_{p_{n}}^{n}\right\|_{\mathrm{L}} \leqslant n^{-1}$ and using (65), we get for any $n \in \mathbb{N}^{*}$,

$$
\left\|f-(\lambda \operatorname{Id}-\mathcal{L}) f_{p_{n}}^{n}\right\|_{\mathrm{L}} \leqslant\left\|f-\left(\lambda \operatorname{Id}-\mathcal{L}^{n}\right) f_{p_{n}}^{n}\right\|_{\mathrm{L}}+\left\|\mathcal{L} f_{p_{n}}^{n}-\mathcal{L}^{n} f_{p_{n}}^{n}\right\|_{\mathrm{L}} \leqslant n^{-1}+\varepsilon_{n} .
$$

Taking the limit $n \rightarrow+\infty$ concludes the proof of (64).

CMla, ens Cachan, CNRS, Université Paris-Saclay, 94235 Cachan, France

Email address: alain.durmus@cmla.ens-cachan.fr

Laboratoire Jacques-Louis Lions and Laboratoire de Chimie Théorique, Sorbonne UniVERSITÉ

Email address: pierre.monmarche@sorbonne-universite.fr

$U R L$ : https://www.ljll.math.upmc.fr/monmarche/

Laboratoire de Mathématiques Blaise Pascal, CNRS UMR 6620, Université ClermontAuvergne

Email address: guillin@math.univ-bpclermont.fr

$U R L$ : http://math.univ-bpclermont.fr/ guillin 
This figure "bounce.png" is available in "png" format from: http://arxiv.org/ps/1807.05421v3 